\title{
A review of argumentation for the Social Semantic Web
}

Editor(s): Harith Alani, The Open University, UK

Solicited review(s): Fouad Zablith, American University of Beirut, Lebanon; Simon Buckingham Shum, The Open University, UK; Iyad Rahwan, Masdar Institute, Abu Dhabi

Jodi Schneider ${ }^{\mathrm{a}, *}$, Tudor Groza ${ }^{\mathrm{a}, \mathrm{b}}$ and Alexandre Passant ${ }^{\mathrm{a}}$

a Digital Enterprise Research Institute, National University of Ireland, Galway, Ireland

E-mail: firstname.lastname@deri.org

b School of ITEE, The University of Queensland, Australia

E-mail: tudor.groza@uq.edu.au

\begin{abstract}
Argumentation represents the study of views and opinions that humans express with the goal of reaching a conclusion through logical reasoning. Since the 1950's, several models have been proposed to capture the essence of informal argumentation in different settings. With the emergence of the Web, and then the Semantic Web, this modeling shifted towards ontologies, while from the development perspective, we witnessed an important increase in Web 2.0 human-centered collaborative deliberation tools. Through a review of more than 150 scholarly papers, this article provides a comprehensive and comparative overview of approaches to modeling argumentation for the Social Semantic Web. We start from theoretical foundational models and investigate how they have influenced Social Web tools. We also look into Semantic Web argumentation models. Finally we end with Social Web tools for argumentation, including online applications combining Web 2.0 and Semantic Web technologies, following the path to a global World Wide Argument Web.
\end{abstract}

Keywords: Argumentation, Semantic Web, Social Web, ontologies

\section{Introduction}

In recent years, the problem of representing largescale argumentation on the Web has attracted the attention of scholars from fields such as artificial intelligence [146], communication theory [2], business management [97] and e-government [116]. At the same time, argumentation researchers began establishing the foundations for a World Wide Argument Web (WWAW) as "a large-scale Web of interconnected arguments posted by individuals to express their opinions in a structured manner" [147].

Arguments on the Web can be used in decisionmaking contexts. Decision-making often requires discussion not just of agreement and disagreement, but also the principles, reasons, and explanations driving

\footnotetext{
${ }^{*}$ Corresponding author.
}

the choices between particular options. Furthermore, arguments expressed online for one audience may be of interest to other (sometimes far-flung) audiences. It can be difficult to re-find the crucial turning points of an argumentative discussion, even one in which we have participated. Yet on the Web, we cannot subscribe to arguments or issues, nor are there tools that support searching for arguments. Nor can we summarize the rationale behind a group's decision, even when the discussion took place entirely in public venues such as mailing lists, blogs, IRC channels, and Web forums.

By providing common languages and principles to model and query information on the Web (such as RDF [99], RDFS [150], OWL [133], SPARQL [177], Linked Data principles [15], etc.), the Semantic Web [17] is an appropriate means to represent arguments and argumentation uniformly on the Web, and to enable, for instance, browsing distributed argumentation 
patterns that appear in various places on the Web. Indeed, researchers have shown that the Semantic Web can be used for visualization and comparison in decision rationale [111].

In this context, this paper discusses research in modeling argumentation as it relates to the Social Semantic Web $[6,24,79]$, focusing on foundational models of argumentation, their applications in the Social Web, and on ontologies (as in computer science [78]).

In particular, our purpose is to investigate ontologies and tools which may be useful for argumentation on the Social Semantic Web, a field where the aforementioned Semantic Web technologies support Social Web [132] applications, while at the same time Social Web paradigms are used to generate Semantic Web data collaboratively and at large scale. This convergence aims at providing new and improved ways to integrate and discover data, following the vision of Social Machines provided by Berners-Lee [16], both on the Web and in the enterprise [137]. In the context of argumentation, this could help to aggregate arguments from various websites - for instance a discussion starting on Twitter and followed up on a mailing list, later frozen on a wiki once consensus is reached - thus providing new means to follow argumentative discussions on the Web. This would enable an argument-centric view of the Web.

Moreover, the Social Web does not yet have widelyused argumentative ontologies, though this problem has been noted [76], along with the need for federation infrastructures [135]. Thus, in order to identify how different argumentation models and tools can be used for the Social Semantic Web, this paper offers a review of more than 150 research papers on the topic, from 1945 to 2011, from which we compare:

- 14 theoretical models of argumentation

- 14 Semantic Web models for argumentation (i.e. ontologies)

- 37 tools for representing argumentation on the Web.

As the focus is on human-centered argumentation [96], with the goal of improving access and providing overviews and visualizations, this article will briefly mention, but not analyze, the agent-based argumentation domain ${ }^{1}$.

${ }^{1}$ See for instance the Argumentation in Multi-Agent Systems (ArgMAS) Workshop series, in its ninth year in 2012. In particular, in the social context, Heras has presented argumentation work from a social perspective using case-based reasoning and ontologies e.g. the ArgCBROntology: http://users.dsic.upv.es/ vinglada/docs/ Sitio_web/ArgCBROnto.html. [82,85]
Following the introduction, we provide brief overviews of argumentation (Section 2.1) and of the Social Web (Section 2.2), then discuss requirements for supporting argumentation on the Social Semantic Web (Section 3). We next present theoretical models of argumentation (Section 4) from a variety of fields, compare them (Section 5), and present applications of these theoretical models (Section 6). Subsequently we present (Section 7) and compare (Section 8) Semantic Web models of argumentation. Then we move on to reviewing tools: in Section 9 we highlight thirteen noteworthy features of Social Web argumentation tools, based on a comprehensive analysis of thirty-seven relevant tools (see the Appendix for full details). Finally we conclude the paper in Section 10.

\section{Background}

\subsection{Argumentation}

Argumentation theory is the study of agreement, disagreement, and of the dialogues and writing through which we convince ourselves and others of our points of view [74]. Informal argumentation occurs throughout conversations, online and offline, often in conjunction with persuasion or with joint decision-making. Even logically sound decisions may involve choices based on values and preference judgements: people may agree on the facts of a situation yet disagree on the preferred outcome or decision to be taken. That is vitally different from disagreeing on the facts of a situation (in which case more information is called for).

We are concerned with argumentative discussions, which we take to be online, mainly textual messages and discussions, in which subjective perspectives or differences of opinion are important and relevant. Groups may use online conversations and social media to coordinate and support decision-making; such argumentative discussions can be found in many online discussion fora such as standardization bodies' listservs, Wikipedia editors' wiki pages, and open source communities' IRC channels, bug reports, and listservs. Individuals may also draw on online conversations and social media in order to form personal opinions and clarify their own preferences, based on sensemaking and analysis of others' experiences; such argumentative discussions can be found, for instance, on product review websites and political blogs, in patient advocacy and support group discussions, and in brief anecdotes shared via microblogs. 


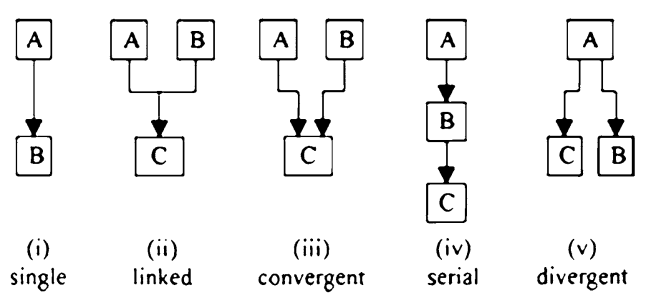

Fig. 1. Common argument patterns, from [143].

There are a variety of common argument structures [143]. A single premise may directly support a conclusion (as in (i) of Fig. 1), but more commonly, they are combined to come to a conclusion. Premises and conclusions may also be chained (as in (iv) of Fig. 1).

Further, as we will see in this review, there are different ways of thinking about and modeling arguments. Argumentation theorists have variously modeled individual argument structure (e.g. Toulmin, discussed in Section 4.1, page 162), argument chaining (e.g. Araucaria $\left.{ }^{2}[152,154,160]\right)$, and groups of arguments (e.g. Dung, discussed in Section 4.5, page 164). There is a significant difference between what is possible to analyze when looking at these different levels of argumentation: they show micro- and macrostructures which are not commensurate, so it is important to have clarity about what kind of analysis suits a situation.

We believe that for social media, the basic units of argumentation are claims and justifications. By a claim, we mean an assertion of fact or opinion ${ }^{3}$. Justifications - reasons for believing the claim - are often elicited when a claim is questioned. Some justifications take the form of explanations; opinions may be elaborated upon and explained even when no disagreement is expected.

\subsection{Social Semantic Web}

The interaction of users around the Web has been shifting from individual siloed Web systems, towards more open and interlinked social applications ${ }^{4}$. In discussion environments, such interlinkage is particularly important: the same community may discuss topics across multiple sites, and use multiple types of sites, such as blogs and microblogs, discussion forums, and

\footnotetext{
${ }^{2}$ http://araucaria.computing.dundee.ac.uk/

${ }^{3}$ A propositional commitment, in Walton and Krabbe's terminology [191].

${ }^{4}$ http://oreilly.com/web2/archive/what-is-web-20.html
}

wikis. Crosslinking the discussions of these systems is a first step, which has been taken by SIOC Semantically-Interlinked Online Communities [23]. Yet the internal structure of these discussions - such as whether the participants agree or disagree, are contributing diverse ideas, or debating in circles - is still not represented in SIOC. Capturing such underlying arguments would be valuable, and research is beginning to address this for instance by identifying argument schemes used in Amazon reviews [84,209] and by modeling the speech acts in Twitter conversations [158]. Yet infrastructure for argumentation on the Social Semantic Web is still needed.

\section{Requirements}

What are the requirements for supporting argumentation on the Social Semantic Web? Arguments must be identified, resolved, represented and stored, queried, and presented to users. Identification involves mining arguments, in the form of claims, from text (Section 6.12.2, page 175), eliciting them from users, or some combination of these approaches. Resolving involves indicating the relationships between the individual claims that make up arguments: are they on the same topic? Do they agree or disagree? Representing and Storing arguments requires a suitable ontology to represent claims and the relationships between them. This supports Querying and enables Presenting the Social Semantic Argument Web, i.e. using these ontologies to facilitate access to conversations, summarizing the contentious and agreed-upon points of a discussion.

The representations chosen are key to this process, since they determine what stored information can be retrieved, and what information needs to be mined and resolved. Existing representations will need to be augmented, since the information we can retrieve depends on what information we store. The desired ontologies should encompass not only the structural features of posts (such as the date and author of a post) and of conversations (such as the reply structure of multiple posts), but also additional argumentative features, for instance to mark claims and to indicate the relationships between them. The simplest relationships for representing argumentation indicate whether pairs of claims support or challenge each other. Yet in general, these relationships do not just pertain to pairs; in general, entire groups of argumentative messages may need to be considered together. The meaning of a dia- 
logue may be lost by chunking messages and treating them individually, out of the original context.

Even simple scenarios may give rise to complex argumentation involving chains of statements (e.g. [184]), and context-dependent relationships in which the conclusion of one argument is premise of another [206]: this makes the graph structures of the Semantic Web a natural fit. Wyner et al. suggest that besides agreement and disagreement, the semantic types of arguments should at least include introduction of a premise or exception, refinement, and pronomial anaphora; they call for a modular architecture "where different relationships or debate components may be added systematically" [206].

\subsection{Example applications \& requirements}

We envision two main approaches to studying argumentation on the Social Semantic Web:

1. Focusing on the real-time, dialogical nature of the Social Web, i.e. by soliciting arguments from humans through conversation and real-time exchange.

2. Focusing on the Social Web as a source of artifacts, i.e. by using existing natural language conversations and reconfiguring the traces and archives of these conversations.

Examples of the first case would be a chatbot or an interactive webform; these could help populate a knowledge base or enable argumentative interaction between humans and intelligent agents. Examples of the second case would be discussion summaries or interactive conversation browsers; a discussion summary could highlight the agreement and disagreement about a topic expressed in a number of Social Web sources, or a review browser could enable faceted navigation through reviews based on the factors they mention, and the polarity and strength of the reviewer's perspective on each such factor.

Formal semantics will be needed in both cases, but for different functions. In the first case, formal semantics translate from natural language to agentappropriate vocabularies, potentially enabling reasoning over human input. Argumentation has long been used for planning between agents: agent-based approaches to the Semantic Web are common [185], and there has been some work in mediating between humans and agents $[175,202]$. In the second case, the semantics will mainly be useful for presenting humans with visualizations and overviews, therefore the ease of mining and presenting representations, and the suitability for human understanding, should be preferred.

Another factor is how - by what process and agent arguments are translated into the formal semantics. This may be the responsibility of a human or machine. If argumentation is annotated by humans - either the person posting a comment or other individuals - they will need sufficient understanding of the model as well as a suitable incentive or motivation for annotating. Meanwhile, machine-based (algorithmic) annotation is limited by our current understanding of informal argumentation, and by the multilayered meaning of conversations. Further, argumentation can be treated as static (for completed discussions) or dynamic (for ongoing conversations). If participants annotate the discussion, the requirement to annotate can distract from the discussion; yet non-participants and machines may be limited by lack of awareness of the context and of subtle language cues.

Models supporting human navigation (e.g. to support our second example above) should make psychological sense; this is not a factor for internal representations for machines. In either case, to ensure feasibility (for either human or algorithmic entry), argumentation classifications need to be clearcut. Thus, the granularity of the model must be limited.

\section{Theoretical models of argumentation}

This section discusses fourteen theoretical models. Seven are designed for capturing argument structure: Toulmin [54], Issue-Based Information Systems [105], Walton's argumentation schemes and critical questions [198], Walton and Krabbe's dialogue types [191], Dung's Argumentation Frameworks [52], Value-based Argumentation Frameworks [12], and Factor and dimension Analysis [13]. An additional seven linguistic approaches deal with issues relevant to argument structure or detection: Speech Act Theory [169], Language/Action Perspective [205], Pragma-dialectic [58], Metadiscourse and Structural Elements of Text [88], Rhetorical Structure Theory [119], Coherence [100], and Cognitive Coherence Relations [162].

\subsection{Toulmin}

The study of informal argumentation originated in philosophy in 1958 with Toulmin [54]. Toulmin sought to find a common underlying basis for arguments in every field of human activity. His model applies, for 


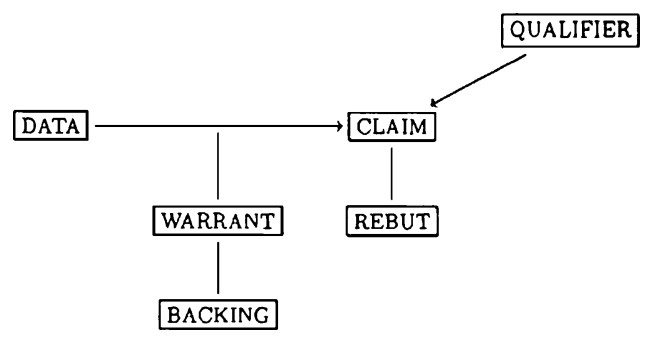

Fig. 2. An interpretation of Toulmin's argument pattern, from [31]

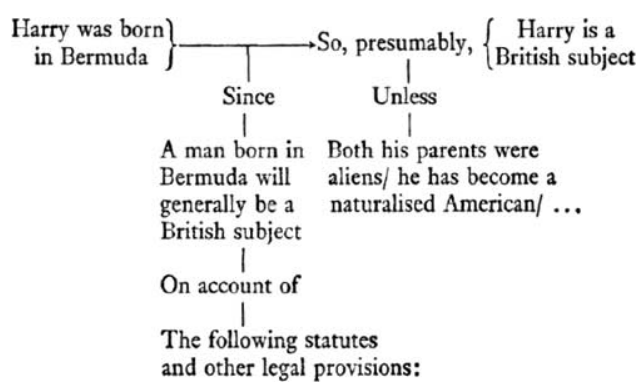

Fig. 3. Toulmin's example argument from page 105 of [54].

instance, to legal, scientific, and informal conversational arguments. In Toulmin's theory, evidence and rules called Warrants support Claims. Claims may also be qualified (i.e. with constraints or to indicate uncertainty); Rebuttals may be used to argue against an argument. Toulmin's argument pattern is shown in Fig. 2: Data is supported by Warrants which have Backings, showing that a Claim holds with Qualifiers regarding the situation, unless there is a Rebuttal. Figure 3 shows Toulmin's now-famous argument, presented according to this structure.

\subsection{Issue-Based Information System (IBIS)}

IBIS, Issue-Based Information System, is a problemsolving structure first published in 1970 [105]. As the name suggests, IBIS centers around controversial issues which take the form of questions. Specialists from different fields may use the same words with different assumptions and intentions ${ }^{5}$, hampering communication. IBIS is especially intended to support community and political decision-making. In this scenario, there may be three separate groups - the participants in the discussion, the relevant experts, and the decision makers - each of whom need to communicate with each

\footnotetext{
5 "Many central terms used are proper names for long stories specific of the particular situation, with their meaning depending very sensitively on the context in which they are used." [105]
}

other and who must also get information from existing records and documentation.

IBIS, as originally designed, is a documentation system, meant to organize discussion and allow subsequent understanding of the decision taken; this explains the use of "Information System" in its acronym. The context of the discussion is a discourse about a topic. Issues may bring up questions of fact and be discussed in arguments. Here, "Arguments are constructed in defense of or against the different positions until the issue is settled by convincing the opponents or decided by a formal decision procedure," [105]. IBIS also recognizes model problems, such as cost-benefit models, that deal with whole classes of problems.

Several kinds of relationships exist between issues: direct successor, generalization, relevant analogy, compatible, consistent, or inconsistent. The method also distinguishes issue content, as factual, deonic ("Shall X become the case?"), explanatory, or instrumental ("Shall we take approach X to accomplish Y?").

Originally implemented as a paper-based system, IBIS influenced several ontologies and numerous tools (see Section 6.2, page 170) as well as procedures such as dialogue mapping [49].

\subsection{Walton's argumentation schemes and critical questions}

The Canadian philosopher Walton has written extensively on argumentation for more than thirty years (e.g. [189,193,194]); a 2010 festschrift honoring his contributions [153] shows how his work has influenced and been applied to computer argumentation. Informal argumentation is one of Walton's specialties [195], and in this section, we discuss two of his key theories, starting with argumentation schemes.

According to Rahwan [143], while many taxonomies of argumentation have been proposed [57,72,94,139], Walton's taxonomy [190] provides the point of departure for computational models of argumentation. In his detailed classification from 1995 [190], Walton describes each scheme with a name, a conclusion, a set of premises, and a set of critical questions. Critical questions address the points where this argument scheme may break down, and suggest attacks against the argument. For example, the following six critical questions are associated with the Argument from Expert Opinion $[71]^{6}$ :

${ }^{6}[71]$ attributes this to page 49, D. Walton, Appeal to Expert Opinion, Penn State Press, University Park, 1997. 


\begin{tabular}{|l|l|l|l|}
\hline \multicolumn{1}{|c|}{$\begin{array}{c}\text { Type of } \\
\text { Dialogue }\end{array}$} & \multicolumn{1}{|c|}{ Initial Situation } & Participant's Goal & \multicolumn{1}{|c|}{ Goal Of Dialogue } \\
\hline Persuasion & Conflict of Opinions & Persuade Other Party & Resolve or Clarify Issue \\
\hline Inquiry & Need to Have Proof & $\begin{array}{l}\text { Find and Verify } \\
\text { Evidence }\end{array}$ & $\begin{array}{l}\text { Prove (Disprove) } \\
\text { Hypothesis }\end{array}$ \\
\hline Discovery & $\begin{array}{l}\text { Need to Find an } \\
\text { Explanation of Facts }\end{array}$ & $\begin{array}{l}\text { Find and Defend a } \\
\text { Suitable Hypothesis }\end{array}$ & $\begin{array}{l}\text { Choose Best Hypothesis } \\
\text { for Testing }\end{array}$ \\
\hline Negotiation & Conflict of Interests & $\begin{array}{l}\text { Get What You Most } \\
\text { Want }\end{array}$ & $\begin{array}{l}\text { Reasonable Settlement } \\
\text { Both Can Live With }\end{array}$ \\
\hline $\begin{array}{l}\text { Information- } \\
\text { Seeking }\end{array}$ & Need Information & $\begin{array}{l}\text { Acquire or Give } \\
\text { Information }\end{array}$ & Exchange Information \\
\hline Deliberation & $\begin{array}{l}\text { Dilemma or Practical } \\
\text { Choice }\end{array}$ & $\begin{array}{l}\text { Co-ordinate Goals and } \\
\text { Actions }\end{array}$ & $\begin{array}{l}\text { Decide Best Available } \\
\text { Course of Action }\end{array}$ \\
\hline Eristic & Personal Conflict & $\begin{array}{l}\text { Verbally Hit Out at } \\
\text { Opponent }\end{array}$ & $\begin{array}{l}\text { Reveal Decper Basis of } \\
\text { Conflict }\end{array}$ \\
\hline
\end{tabular}

Fig. 4. Walton's seven types of dialogue, from [196].

1. How credible is $E$ as an expert source?

2. Is $E$ an expert in the field that $A$ is in?

3 . Does $E$ 's testimony imply $A$ ?

4. Is $E$ reliable?

5. Is $A$ consistent with the testimony of other experts?

6. Is $A$ supported by evidence?

Walton's 2008 book [198], coauthored with computational argumentation researchers, presents 65 general argumentation schemes, presumably updating [190].

\subsection{Walton and Krabbe's dialogue types}

Discussion types, first developed by Walton and Krabbe [191], have also been influential. ${ }^{7}$

Seven types of dialogue are shown in Fig. 4. These types are Persuasion, Inquiry, Discovery, Negotiation, Information-Seeking, Deliberation, and Eristic. They are distinguished by the initial situation, the individual goals of the participants, and the overall goal of the dialogue. For instance, an information-seeking dialogue and an inquiry have similar goals, but differ in the initial situation: one person is believed to have the answer in an information-seeking dialogue, while in an inquiry, no one has the answer. Persuasion and deliberation are distinguished by whose preferences are used: in a persuasion dialogue, the outcome depends on the preferences of the individual to be persuaded, while in a deliberation, the group preferences are used.

\footnotetext{
${ }^{7}$ Walton has revised this taxonomy several times. 'Discovery' was not in several earlier formulations, such as [193] (p. 183); it is motivated by choosing the best hypothesis for testing. Debate and Pedagogical appeared in an earlier formulation [192] which provides descriptions of the goals of each dialogue. Other scholars have also suggested extensions and modifications, for instance Dunne et al. have proposed adding examination dialogues [53].
}

Understanding the goal of a conversation is important for determining the outcome, and for determining what conversational moves are relevant. We may be able to access to pragmatics [101] of a conversation by understanding its goals. This is also how we evaluate a conversation: What was A trying to achieve? What was B trying to achieve? Did they achieve it?

In our own view, these types of dialogue can be classified based on whether knowledge plays a large, middling, or minor role. Inquiry, Discovery, and Information-seeking dialogues are almost entirely knowledge-based, while knowledge plays only a minor role in Negotiation (aiming at a harmonious settlement) and Eristic (quarrels, beneficial mainly for venting emotions). Knowledge plays some role in the remaining two types: in Persuasion and Deliberation, opinion and belief also have a large role. Further complexity arises because dialogue types may shift in an actual discussion, and argument schemes may be embedded in one another [196].

\subsection{Dung's argumentation frameworks}

Dung provides a powerful graphical model of argumentation frameworks in [52], which has been widely used in computational argumentation. Argumentation frameworks are defined as sets of arguments and attacks between them. Formally, an argumentation framework is a pair $A F=\langle A R$, attacks $\rangle$ where $A R$ is a set of arguments, and attacks is a binary relation on $A R$, i.e. attacks $\subset A R \times A R$.

Then the questions of interest are to find maximal sets of arguments that do not attack each other (these are called conflict-free), and to find arguments that are not defeated by a given set of arguments (these are called acceptable). A conflict-free set of arguments is 


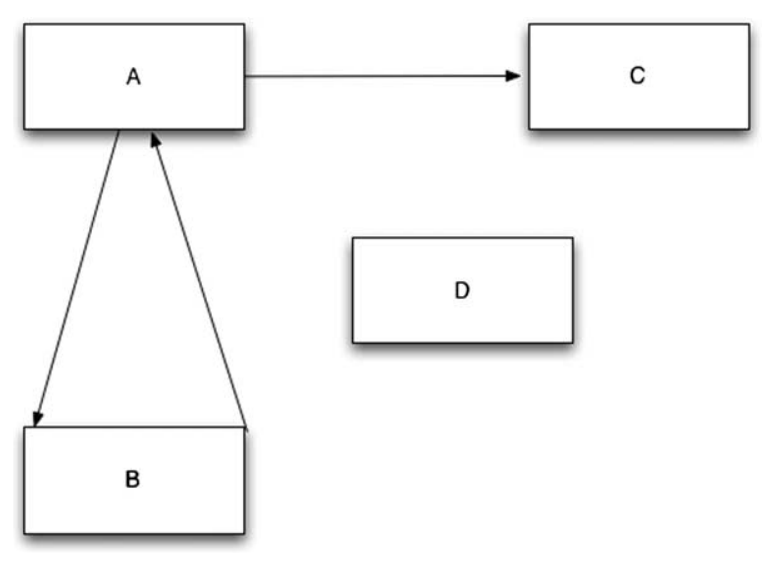

Fig. 5. Example of an argumentation framework.

then considered to be admissible if each argument is acceptable with respect to the set.

Dung then finds maximal admissible sets, known as preferred extensions. Also important are the grounded extensions, which represent the least fixed point of the function mapping an argumentation framework to the set of acceptable arguments of that framework. A stable extension is a conflict-free set of arguments that attacks each argument that does not belong to the set.

A simple argumentation framework is shown in Fig. 5. In this example, $A$ and $B$ attack each other; A also attacks C; and D is not attacked. Thus A, D and $\mathrm{B}, \mathrm{C}, \mathrm{D}$ are preferred extensions.

In Dung's theory, there is a notion of 'defend' - to defeat the attackers - but there is no direct notion of 'support'. Arguments 'support' another one by not being defeated, and by not attacking, a given argument.

\subsection{Value-based Argumentation Frameworks}

Value-based Argumentation Frameworks [12], based on Dung's argumentation frameworks, address persuasion and preferences. It is not just differences about the facts, or failures in logic that can cause reasonable people to disagree: differences in values can also be to blame. For instance: "Despite the fact that the weather is beautiful, I choose to stay inside, because I have something important to do". In practical reasoning, two people can come to different, consistently logical opinions, based on a difference of values: "A key element in persuasion is identifying the value conflict at the root of the disagreement so that preference between values can explicitly inform the acceptance or rejection of the competing arguments." The theory of Valuebased Argumentation Frameworks thus draws from Perelman's notion of audience [139]: arguments are often addressed to particular audiences, and persuasive arguments are those aligned with the audience's values and preferences. Value-based Argumentation Frameworks provide a method for logically calculating consistent approaches, distinguishing between the facts of a situation and community members' values.

\subsection{Factor and dimension analysis}

There is a large business market in legal information retrieval, and one method for classifying and indexing legal cases has been the key factors of a case, for instance for the early legal argumentation system CATO [3]. Factor analysis can be helpful in supporting community decision-making or in summarizing reviews. Factors are simplifications that are either present or absent; when present, a factor "always strengthens the case for the same disputant" [13].

Further development along the lines of CATO yielded Ashley's later system, HYPO [7], which uses dimensions rather than factors. Dimensions "capture the legal relevance of a cluster of facts to the merits of a claim". Dimensions such as "Obligation to aid the victim" or "Failure to heed traffic signs" contribute to determinations of culpability, and have been recorded in manually constructed databases [8]. Dimensions can be present to a greater or lesser degree and it may unclear which side they favor.

\subsection{Speech Act Theory}

Several approaches to conversation and argumentation have been derived from Speech Act Theory. Searle's Speech Act Theory [169] describes five categories of speech acts: assertives, directives, commissives, expressives, and declaratives. Speech acts are about the force of a statement: what effect they seek to have on the hearer or the world. Assertives ('The sky is blue') assert that something is true. Directives ('Clean your room') order, permit, or request something. Commissives are vows or pledges ('I swear to tell the truth'). Expressives offer thanks or congratulations, or express feelings ('Great work!'). Declarations ('I now pronounce you man and wife') enact what they say, effectively changing reality. ${ }^{8}$ Speech Act Theory is not a complete model of argumentation, yet it is a relevant theory that has been widely influential. For example, earlier (Section 4.4, page 164) we discussed that understanding the goals of a conversation could be

\footnotetext{
${ }^{8}$ As with all speech acts, sincerity is a criterion, and social criteria, e.g. ceremony, may also hold.
} 
important for interpreting it; the same speech acts can be used in different ways, depending on the goals of a dialogue ${ }^{9}$.

\subsection{Language/Action Perspective}

The Language/Action Perspective (LAP) [205] embeds Speech Act Theory in a task-based framework. Argumentation is found in each of the three types of conversations which accomplish goals in the Language/Action Perspective, according to de Moor and Aakhus: Conversations for action involve making commitments; conversations for possibility create a context for action; and conversations for disclosure allow participants to share their views and concerns [128].

\subsection{Pragma-dialectic}

The pragma-dialectic approach is a complete argumentative theory, which has been developed over a number of years in numerous scholarly works (especially [56-58]) and popularized in the authors' textbooks (e.g. [59]). Like the Language/Action Perspective, it uses speech acts, further developing Searle's theory in order to model argumentation. Rather than focusing on the logical forms and patterns of reasoning, as Walton does, van Eemeren and Grootendorst's pragma-dialectic theory views argumentation as a social process, used to settle "a difference of opinion by verbal means" [59] (pp. ix-xii).

Depending on the context, the same speech acts can function as an explanation, a piece of information, or as an argumentation: for argumentation, the context must include a difference of opinion. Depending on their order and position in the discussion, speech acts take on different meanings, as we will see in Fig. 6. Further, Searle's illocutionary speech acts, which function at the sentence-level, combine in argumentation into a higher-order textual element: the argumentation is itself seen as a complex speech act.

The main speech acts within an argumentation are assertives, commissives, and directives. Expressives which express emotions - do not help resolve the difference of opinion, but may affect how or whether the discussion proceeds. Declaratives - which bring a state of affairs into being - are relevant for definitions, specifications, amplifications, and explanations; van

\footnotetext{
${ }^{9}$ For a pertinent example see the persuasion and deliberation scenarios discussed in Atkinson et al. [9].
}

\begin{tabular}{|c|c|}
\hline I & Confrontation \\
\hline Assertive & Expressing a standpoint \\
\hline Commissive & $\begin{array}{l}\text { Acceptance or non-acceptance of a standpoint, } \\
\text { upholding non-acceptance of a standpoint }\end{array}$ \\
\hline [Directive & Requesting a usage declarative] \\
\hline [Usage declarative] & Definition, specification, amplification, etc.] \\
\hline II & Opening \\
\hline Directive & Challenging to defend a standpoint \\
\hline Commissive & $\begin{array}{l}\text { Acceptance of the challenge to defend a standpoint } \\
\text { Agreement on premises and discussion rules } \\
\text { Decision to start a discussion }\end{array}$ \\
\hline [Directive & Requesting a usage declarative] \\
\hline [Usage declarative] & Definition, specification, amplification, etc.] \\
\hline III & Argumentation \\
\hline Directive & Requesting argumentation \\
\hline Assertive & Advancing argumentation \\
\hline Commissive & Acceptance or non-acceptance of argumentation \\
\hline [Directive & Requesting a usage declarative] \\
\hline [Usage declarative] & Definition, specification, amplification, etc.] \\
\hline IV & Concluding \\
\hline Commissive & Acceptance or non-acceptance of a standpoint \\
\hline Assertive & $\begin{array}{l}\text { Upholding or retracting a standpoint } \\
\text { Establishing the result of the discussion }\end{array}$ \\
\hline [Directive & Requesting a usage declarative] \\
\hline [Usage declarative] & Definition, specification, amplification, etc.] \\
\hline
\end{tabular}

Fig. 6. Distribution of speech acts in a critical distribution from [58].

Eemeren and Grootendorst call these "usage declaratives".

The pragma-dialectic approach also stresses the principles of clarity, honesty, efficiency, and relevance, updating Grice's Cooperation Principle [73] - which focuses on the intention of language - with the Searlean focus on the communicative aspects of language use. Relevance, for example, can be global, local, subject matter-specific, or probative. An argument may be relevant at one phase, but irrelevant at another; for example an argument related to selecting the topic of discussion is not relevant once the topic has been agreed upon.

To understand the force of a speech act - whether an assertive, commissive, or directive - we must identify where we are in the argumentation. van Eemeren and Grootendorst identify four dialectical stages of argumentation: Confrontation, Opening, Argumentation, and Closing [58]. In the confrontation stage, the issue at hand is announced, agreed upon, or clarified. In the opening stages, the rules are agreed to (perhaps implicitly). In the main stage (Argumentation), each party is expected to make a serious effort to support his point of view, while also allowing the other party to make his case. Finally, the argument closes when the goal is fulfilled or the parties agree to end the debate.

The pragma-dialectic approach is far more extensive in attending not only to linguistic patterns, but also to the social context in which they are embedded (i.e. 
the linguistic pragmatics, see e.g. [101]). Argumentative discourse starts with the assumption that the listener does not (necessarily) agree with the speaker's position, and aims at "coming to a reasonable agreement" [59] (p. 4). Speakers may anticipate objections, explaining their reasoning to account for expected (implicit) differences of opinion. Or, they may wait to hear their conversational partners' standpoints or doubts, and then respond.

The issue, according to the pragma-dialectic theory, may be single or multiple, and mixed or nonmixed. Single disagreement is about just one proposition while multiple disagreement is about more than one proposition. If both a positive and a negative standpoint are taken on the issue, the disagreement is mixed, otherwise it is simple. This is a particularly useful distinction for conversations in social media.

Pragma-dialectic theory is also particularly useful for determining which parts of social media discourse can be considered argumentative, since it presents phrases that tend to mark argumentation, and since it treats speech acts from an argumentative perspective. For example, doubt is often implicit, but certain phrases mark it more explicitly, such as "I don't know whether", "I'm not yet convinced that", "Couldn't it be that", and "I'll have to think about whether".

Similarly, set phrases often indicate the topics of a debate - helping us detect a participant's standpoint, which "expresses a certain positive or negative position with respect to a proposition" [58] (p. 3). Standpoints may often be obliquely stated, yet they can sometimes be recognized by the appearance of particular phrases, which can be baldly stated ("my standpoint is that", "we are of the opinion that") or explicit ("I think that", "if you ask me", "therefore"). Some phrases that may be used to indicate a standpoint also permit alternate interpretations ("the way I see it", "in other words", "all things considered"). Other patterns, like "shouldn't", "you must never", "that. .. is", "ought to be", commonly co-occur when a standpoint is expressed [59] (pp. 10-12).

Such phrases, like the metadiscourse markers we discuss next, can potentially be applied in detecting and mining argumentation (which we later discuss in Section 6.12.2, page 175). However, phrase-detection alone does not suffice, and significant challenges remain. Language is multivalent and context is needed for properly reconstructing argumentation. "In language use there is often the case that there is more than one purpose at the same time, and if language is used argumentatively, the argumentative function need not always be the most important," [58] (p. 23), meaning that reconstructing argumentation must extract a single thread of meaning out of many.

\subsection{Linguistic markers of argumentation: Metadiscourse and structural elements of text}

We next discuss linguistic markers of argumentation; strictly speaking these are not theoretical models of argumentation, yet they identify possible argumentation, essentially enabling the argumentation structures to be annotated in and abstracted from the text.

Metadiscourse refers to the "aspects of a text which explicitly organize a discourse or the writer's stance towards either its content or the reader." [88] (p. 14). Argumentative words and phrases such as 'but' and 'according to $\mathrm{X}$ ' are prominent examples. Metadiscourse is used not only to structure text but also to influence the reader's view.

Hyland classifies metadiscourse into interactive and interactional types. Interactive metadiscourse, which organizes text, includes transitions (in addition, but, thus, and); frame markers (finally, to conclude, my purpose is); endorphoric markers (noted above, see Fig, in section 2); evidentials (according to $X, Z$ states); and code glosses (namely, e.g., such as, in other words) [88] (p. 49). Interactional metadiscourse, which makes the author's views explicit and invites readers' response, includes hedges (might, perhaps, possible, about); boosters (in fact, definitely, it is clear that); attitude markers (unfortunately, I agree, surprisingly); self mentions (I, we, my, me, our); and engagement markers (consider, note, you can see that).

As markers of the persuasive and rhetorical elements of texts, metadiscourse elements are likely to be useful signals for identifying claims and arguments in social media.

\subsection{Rhetorical Structure Theory}

Rhetorical Structure Theory (RST) [119], a method for analyzing texts according to their structure and rhetorical role, was developed at the University of Southern California's Information Sciences Institute to assist with computer-based text generation. In RST, structures such as 'Concession', 'Evidence', and 'Justify', called 'relations', describe the relationship of two or more spans of text. Generally one span (the most important) is called the nucleus, while the less important spans are known as satellites. In some situations (such as sequences and contrasts), both spans are nuclei of 
equal weight. Justifications and hedges are more likely to appear in satellites while the nucleus is more likely to contain claims; this has potential application in detecting arguments and in summarizing Social Web applications.

\subsection{Coherence}

Coherence is another important concept in text and dialogue. Coherence is not an argumentation theory per se, but it is both an essential part of text, and an essential part of argumentation, since before an argument can be understood (much less formally evaluated), how its parts hold together or interrelate must be understood. Knott compares the following two examples [100].

1. "Tim must love that Belgian beer. The crate in the hall is already half empty."

2. "Tim must love that Belgian beer. He's six foot tall."

While the first example is coherent the second example is more challenging to make sense of: the reader expects (but does not get) a sensible explanation or evidence for why Tim must love that Belgian beer.

Argumentation relies on coherence: Adding 'because' works in the first example but not in the second example. The word 'because' stresses the expected causal relationship, making the informal argument more evident.

3. "Tim must love that Belgian beer, because the crate in the hall is already half empty."

In Sentence 3, the reader must still infer some information, such as that the crate in the hall contains Belgian beer, and that Tim is the main person drinking the contents of the crate. Such missing premises are typical in informal argumentation.

\subsection{Cognitive Coherence Relations}

One actionable way of expressing coherence is by using specific signaling terms. The causal relationship (expressed in 'because') is one of the Cognitive Coherence Relations which Sanders uses to explain how readers understand text [162]. The four Cognitive Coherence Relations are: Basic Operation (causal or additive), Source of Coherence (semantic or pragmatic), Polarity (positive or negative), Order of Segments (for causal relations only: basic or non-basic, depending on whether or not the antecedent appears before the con- sequent). Based on this, the relationship signaled in because in Sentence 3 (above) is causal, pragmatic, positive, and basic.

\section{Comparison of theoretical models}

Any of these models could be expressed in semantic formats (e.g. RDF) since they are compatible with a graph-based representation of argumentation. Yet for modeling argumentation on the Social Semantic Web, it is most meaningful to examine the challenges and opportunities that might advantage any one model or framework over the others. As previously noted, various units have been modelled - individual argument structures (e.g. Toulmin, discussed in Section 4.1, page 162), argument chaining (e.g. Araucaria $\left.^{10}[152,154,160]\right)$, and groups of arguments (e.g. Dung, discussed in Section 4.5, page 164).

We can make several further distinctions between models, for instance based on the community in which they originated, their purpose or use, the extent to which they focus on disagreement, the unit of analysis on which they focus, their granularity, and their suitability for automation or for aiding human reasoning.

\subsection{Community of origin}

Various communities have contributed models, particularly the argumentation and linguistics communities. The IBIS model comes from management and was later taken up by design rationale and humancomputer interaction (HCI) communities. The Language/Action Perspective originated in artificial intelligence and HCI and was later adopted by communication theorists. In some cases, models bridge communities: the pragma-dialectic approach is an argumentation model which has been heavily influenced by linguistics, and by the theory of pragmatics [101] in particular. Models have been shaped by their originators and proponents, and the purposes for which they were intended.

\subsection{Purpose or use}

The intended purpose for each model depends largely on its origin. Models put forth by the argumentation community are generally designed to support either analysis (e.g. to determine the reasoning

\footnotetext{
${ }^{10} \mathrm{http} / / /$ araucaria.computing.dundee.ac.uk/
} 
patterns used and to identify fallacies) or formal reasoning, in order to address questions such as computational decisions of which argument won, what the deciding factors were, or what values and preferences were expressed in the discussion. Models of linguistic features may be used in discourse analysis, for summarization, and to support natural language generation by both machines and non-native speakers. Models from other communities are generally intended to support flow-based process analysis, for instance to organize information in order to avoid information overload, to speed human decision-making, and to provide a record of collaborative thought processes.

\subsection{Agreement/disagreement focus}

Disagreement and the process of coming to consensus are the core of argumentation. While disagreement and agreement are central in models coming from the argumentation community, other models focus on this core to a greater or lesser extent. Most linguistic models are considerably broader and less focused on the argumentation aspects, yet in addressing conversation, they provide valuable insights as well as analysis tools. HCI models focus on supporting collaboration and shared visions; disagreement is analyzed or understood only to the extent necessary for coming to consensus or providing an overview of viewpoints.

\subsection{Unit of analysis}

Different units of analysis have been used. At the language layer, models may focus on the relationship between different clauses (Metadiscourse, Coherence, Cognitive Coherence, RST) or the communicative function of different words, phrases, and sentences (Speech Act Theory and the Language/Action Perspective). The pragma-dialectic approach focuses on the propositional level, while factors analysis looks at important attributes or dimensions. Other models focus on classifying individual arguments and their relation to a whole (IBIS), or studying the internal structure of arguments (Toulmin, Walton, Argumentation Frameworks, Value-based Argumentation Frameworks).

\subsection{Granularity}

Models of linguistic features are more granular, but sometimes less focused on the overall structure. Coarse-grained and simple models, such as IBIS, more common in application. Yet even IBIS is not gener- ally applied in its full complexity, but is rather reduced to focusing identifying issues, and then on identifying pros and cons for a particular issue. Fuller versions become more complex by looking at the relationships between arguments: what responds to what.

\subsection{Ease of automated application}

Mechanistic application is possible for some models but not for others. In particular, classification for Walton's model would be quite difficult due to the large number (65) of argument categories and the need for detailed reasoning. On the hand, in many cases language can be mechanically analyzed. Identification and classification of argumentation via language technologies is still in its infancy, yet offers great potential to expand algorithmic understanding of language.

\subsection{Support for human reasoning}

To aid human reasoning, however, linguistic models that mainly use cue words are probably too granular since they occur at the sentence level, probably more granular than needed. For this purpose, Walton's critical questions are very useful, because they can point humans to the questions that need to be addressed, opening the door to checklists for reasoning, which could be applied consistently by groups. Value-based frameworks also address the basic reasoning underlying social decisions: each person has their own reasons, which may be revealed in the course of a discussion. Focusing on what the values are, and being able to articulate them, can help both in developing empathy for dissenting viewpoints, and in making clear the rationale for group decisions when consensus is needed.

\section{Applications of the models to the social and Semantic Web}

The theoretical models discussed have been quite influential, and in many cases we can directly trace Social and Semantic Web applications of these models. In this section we describing some applications, reviewing each of the models in turn.

\subsection{Applications of Toulmin}

Toulmin is cited frequently and in numerous fields, from rhetoric (e.g. [199]) to education (e.g. [38]) to 


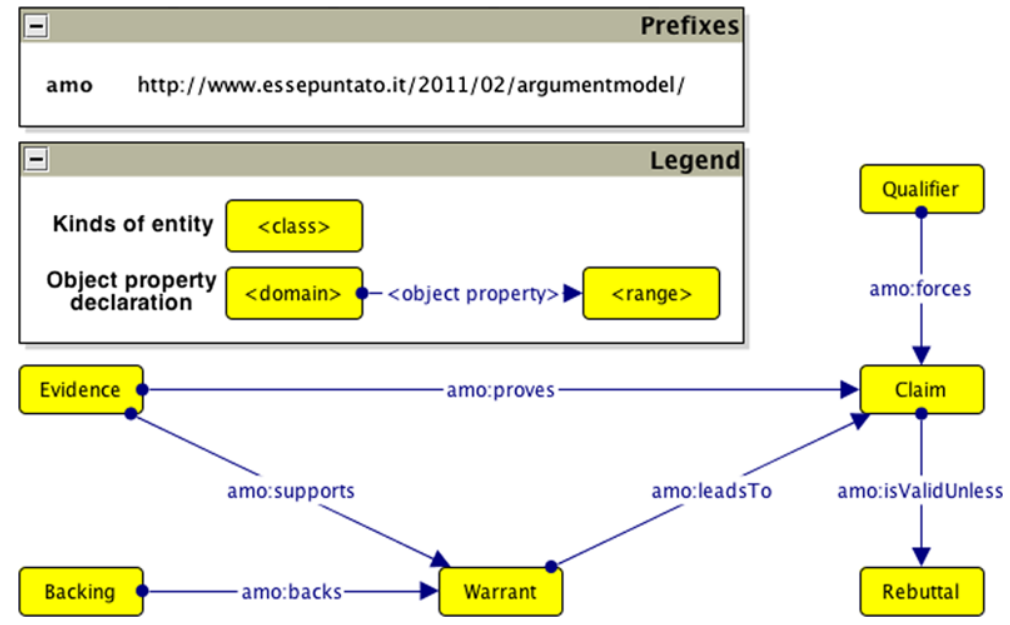

Fig. 7. The core Toulmin argument model ontology from [140].

computer argumentation (e.g. [112]). While his model is a useful abstraction, scholars have argued about whether people actually think in terms of Toulmin's warrants [130].

One early hypertext system, SEPIA, drew from the Toulmin system [178]. A modified version of Toulmin's argumentation schemes have been used to describe a cooperative dialogue game, implemented in Prolog, in which the participants' goal is to reach a claim on which they agree, while also producing a supporting argumentation structure for the claim [11]. Such dialogue games could be modified for use in mixed-initiative systems.

In the Semantic Web, the Toulmin Argument Model has been implemented by an OWL 2 DL ontology that imports CiTO $^{11}$ [140]. It follows Toulmin's model closely, as shown in Fig. 7.

\subsection{Applications of Issue-Based Information System (IBIS)}

Although many tools are described as 'using the IBIS model' or 'IBIS-like', there is significant variation in the underlying structure of these models [91]. In our view, these models use 'IBIS-like' to mean that they concern decision-making or design rationale, provide graphical representations, and use some form of polarity.

The IBIS model has a long history of use, particularly with early hypertext systems. Early critiques of IBIS came from the design rationale community. One difficulty was that only deliberated issues were

\footnotetext{
${ }^{11} \mathrm{http}: / / \mathrm{www} . e s s e p u n t a t o . i t / 2011 / 02 /$ argumentmode
}

included; Procedural Hierarchy of Issues (PHI) modifies IBIS to allow inclusion of subissues which are not deliberated [64]. PHI was adopted by another early system, the Author's Argumentation Assistant [168], which also drew from the authors' earlier Toulminbased system, SEPIA [178].

Another difficulty, representing the relationships and interdependencies of issues [64], remains challenging to resolve, though ideas such as nonfunctional requirements and dependencies (Section 7.6, page 178), might be relevant.

IBIS has also been used outside of design rationale. For instance, Gerosa et al. [68] discuss an elearning message board system adopting a modification of IBIS, where message types are specified. In addition to the IBIS-analogues, Question, Argumentation, and Counter-Argumentation, the system adds two types: Seminar (a general topic for the week) and Clarification. IBIS has also influenced the design of modern ontologies, including the SALT Rhetorical Ontology, SIOC-Argumentation, DILIGENT, and the Change Ontology which we discuss after reviewing IBIS' RDF representation.

\subsubsection{IBIS RDF}

IBIS $\mathrm{RDF}^{12}$ is an RDF representation of the IBIS model. refersto is modelled as a subProperty of dcterms: reference with two subproperties, pro and con. The larger IBIS vocabulary provides Published Subject Indicators ${ }^{13}$ for important terms, in-

\footnotetext{
${ }^{12} \mathrm{http} / / /$ purl.org/ibis

${ }^{13} \mathrm{http}: / /$ www.topicmaps.org/xtm/index.html\# def-published-subject-indicator
} 


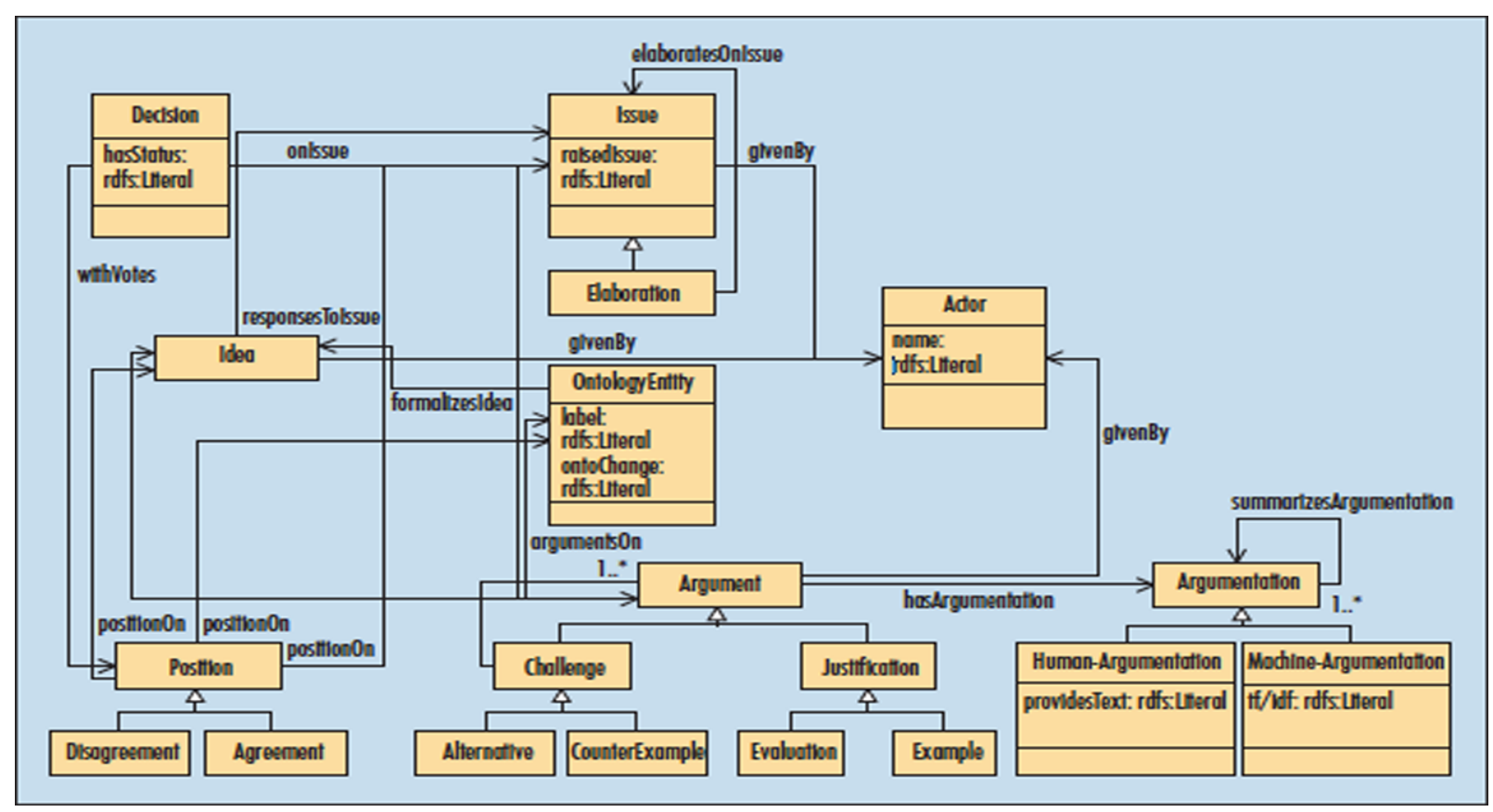

Fig. 8. An overview of the core DILIGENT ontology from [181].

cluding pro, con, Idea, Question, Argument, Decision, and Reference.

\subsubsection{SALT rhetorical ontology}

SALT [77] is a rhetorical ontology for scholarly communication. In SALT, opposing arguments can be connected together with the relation hascounterArgument, while a RhetoricalElement can also be connected with what it argues for (Argument and has Argument, for instance). SALT's argumentation also includes Reason, which contains Argument (further specified to PositiveArgument or NegativeArgument) and CounterArgument.

\subsubsection{DILIGENT ontology}

DILIGENT provides an argumentative structure for collaborative ontology construction; the acronym comes from the phrase DIstributed, Loosely-controlled and evolvInG Engineering processes of oNTologies. DILIGENT draws from both RST (Section 4.12, page 167) and IBIS (Section 4.2, page 163), as shown in Fig. 8.

\subsubsection{Change Ontology (ChAO) in Collaborative Protégé}

DILIGENT [67] itself influenced the Change Ontology in Collaborative Protégé. Castro et al. distinguish between an argument (which is well-focused and specific) and an elaboration (which provides sup- port for the argument, possibly with file attachments) [67]. Positions become clear through the disputeresolution process. With Protégé, various argumentation-related Annotations can be added, including Explanation, Proposal, and AgreeDisagreeVote [167].

\subsubsection{SIOC-Argumentation}

The SIOC-Argumentation ${ }^{14}$ model [107] expands on the IBIS model with terms such as Decision and Argument. It is provided as an extension of SIOC Semantically-Interlinked Online Communities [23] a model that focuses on representing online communities and the content shared within them.

While SIOC simply focuses on the notion of replies (sioc:reply_to) to represent connections between discussion items, the SIOC-Argumentation module goes further and provides finer-grained representation of discussions and argumentations in online communities.

So far a modification of SIOC that draws from DILIGENT and OMDoc has been used in the math wiki system SWiM $^{15}$ [108], a Semantic Wiki for Mathematical Knowledge Management. The SIOC/ OMDoc argumentation ontology (Fig. 9 on page 172)

\footnotetext{
${ }^{14}$ http://rdfs.org/sioc/argument

${ }^{15} \mathrm{http}: / / \mathrm{kwarc}$. info/projects/swim/demo.html
} 


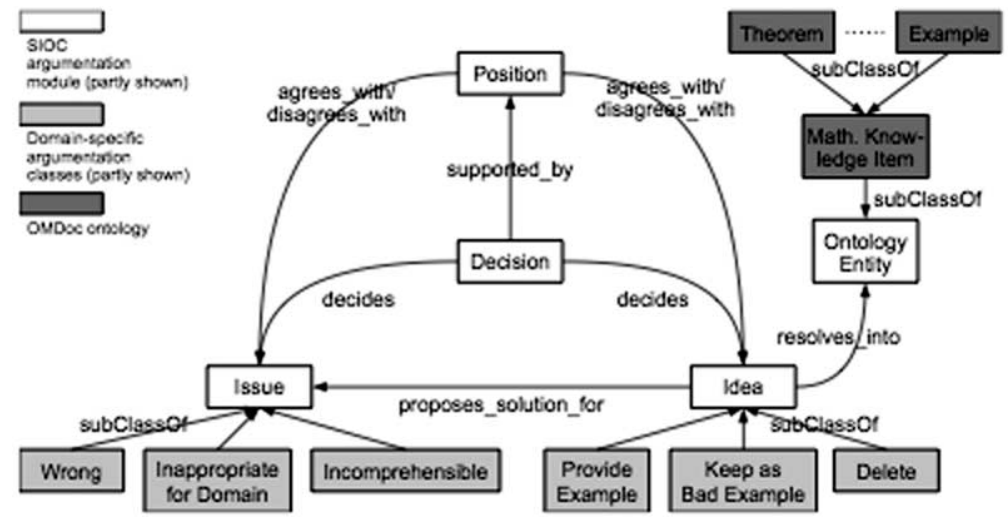

Fig. 9. The argumentation ontology from SWiM extends SIOC-Argumentation and DILIGENT Argumentation [108].

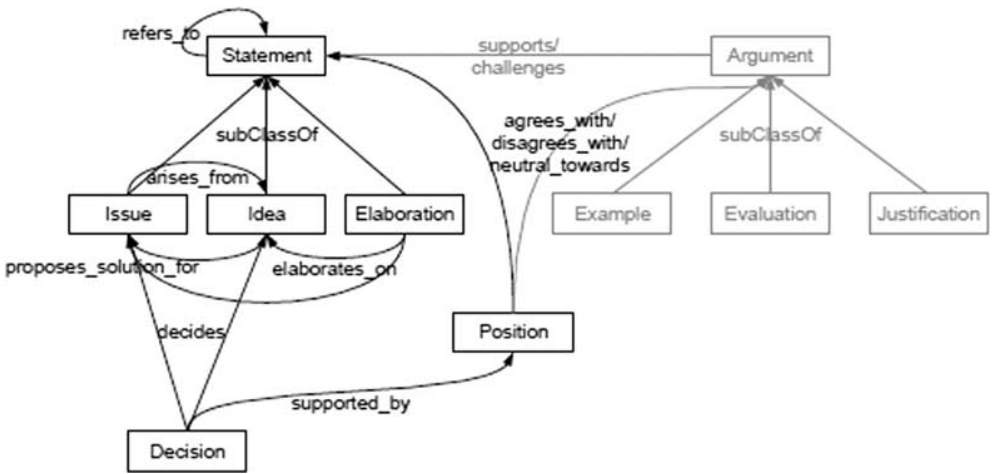

Fig. 10. An overview of the SIOC-Argumentation module from [107].

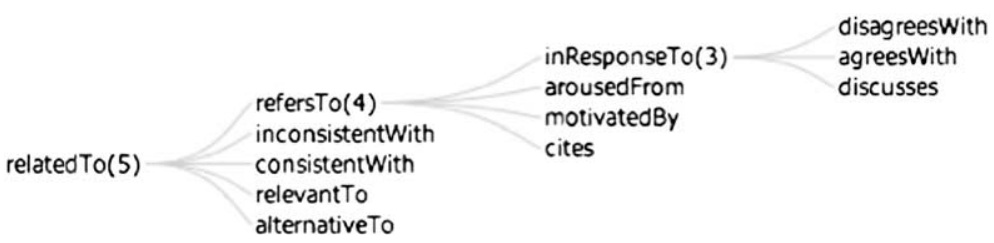

Fig. 11. SWAN-SIOC ontology from [44].

is further described in Lange's dissertation [106]. It incorporates IBIS-style classes from SIOC (Position, Decision, Idea, and Issue), as well as domainspecific argumentation classes for math (e.g. Wrong, Keep_as_Bad_Example, Incomprehensible).

As opposed to IBIS-RDF, SIOC-Argumentation (Fig. 10) provide the means to easily integrate argumentation modelling patterns with Social Web applications since it relies on SIOC, already used in various applications (Drupal7, etc.). However, SIOCArgumentation has limitations: it does not represent taxonomic, causal, or similarity relations, which prevents its use in contexts that require deeper analysis of arguments.

\subsubsection{SWAN-SIOC}

SWAN-SIOC [138] harmonizes the argumentation aspects of two pre-existing ontologies: along with SIOC, it is based on SWAN - Semantic Web Applications in Neuromedicine [46] - an ontology which focuses on scientific communication in neurology.

SWAN/SIOC uses twelve terms, as shown in Fig. 11. The most general term is relatedTo, which has five direct descendents or subterms. These, in turn, may have subterms, until we reach the base terms in the ontology: disagreesWith, agreesWith, and discusses. SWAN/SIOC provides a simple model for the relationships between items. Tools using 
SWAN-SIOC include PDOnline, which is discussed in Section A.31, page 210 .

\subsection{Applications of Walton}

Walton's model has been widely applied in computational argumentation [153], and recent research has demonstrated how argument schemes could be used to aid sensemaking in Amazon reviews [83,84,209]. Avicenna and ArgDF incorporate Walton's schemes. The only Social Web application we are aware of is Parmenides $^{16}$ [35-37], which uses the following argumentation scheme and Value-based Argumentation Frameworks [12]:

Argumentation Scheme AS1:

- In the current circumstances $R$,

- we should perform action $A$,

- to achieve new circumstances $S$,

- which will realize some goal $G$,

- which will promote some value $V$.

The following sixteen critical questions are associated with Argumentation Scheme AS1:

CQ1 Are the believed circumstances true?

CQ2 Assuming the circumstances, does the action have the stated consequences?

CQ3 Assuming the circumstances and that the action has the stated consequences, will the action bring about the desired goal?

CQ4 Does the goal realize the value stated?

CQ5 Are there alternative ways of realizing the same consequences?

CQ6 Are there alternative ways of realizing the same goal?

CQ7 Are there alternative ways of promoting the same value?

CQ8 Does doing the action have a side effect which demotes the value?

CQ9 Does doing the action have a side effect which demotes some other value?

CQ10 Does doing the action promote some other value?

CQ11 Does doing the action preclude some other action which would promote some other value?

CQ12 Are the circumstances as described possible?

CQ13 Is the action possible?

CQ14 Are the consequences as described possible?

CQ15 Can the desired goal be realized?

CQ16 Is the value indeed a legitimate value?

\footnotetext{
${ }^{16}$ http://cgi.csc.liv.ac.uk/ parmenides/
}

\subsection{Applications of Walton \& Krabbe's dialogue types}

Walton \& Krabbe's Dialogue Types have been influential. Persuasion, in particular, has been extensively studied [142], and the wider area of dialogue games has been an active area of agent argumentation [122]. In applications to human argumentation, some work on deliberation (e.g. [197] and the Parmenides system Section A.30, page 210 as a whole) has used dialogue type to focus the discussion.

\subsection{Applications of Dung's argumentation frameworks}

Dung's argumentation frameworks have been incredibly influential: as of 2011, the original 1995 paper has over 450 citations in the ACM Digital Library, and over 1500 in Google Scholar. A Java reasoner called Dungine implements Dung's acceptability semantics [176]. Dungine is part of $\mathrm{ArgKit}^{17}$, a Java 5 development toolkit for applications that use argumentation; it is open source, licened under LGPL.

Dung's approach has driven computational research in argumentation and provided the basis for a large body of theoretical work. Among theoretical extensions of Dung's work, we have focused on Value-based Argumentation Frameworks, and the relevant Social Web application of Dung is in fact an application of that extension, as we next explain.

\subsection{Applications of Value-based Argumentation Frameworks}

Parmenides uses value-based argumentation frameworks in addition to the argument scheme and critical questions discussed above. It pinpoints the source of the disagreement, by having participants respond to a series of questions in a survey format. The group's preferences are revealed in the results, which are displayed to administrators as graphical argumentation frameworks.

\subsection{Applications of factor analysis}

Factor analysis has been applied in Ashley's legal argumentation systems, but we know of no specific Social Web applications.

\footnotetext{
${ }^{17}$ http://argkit.org/
} 
The factors analysis approach is still used by commercial providers of legal information [208]. More recently, automatic text mining has been used to identify these factors [208]. Generalizing factors, perhaps using argument schemes and critical questions, could provide another approach to argument mining; see for instance Heras' manual application of argument schemes to Amazon reviews [84] and Schneider's factors analysis of Wikipedia deletion discussions [165].

\subsection{Applications of Speech Act Theory}

Speech acts are used to model the flow of online conversation in several recent works. Jeong et al. [92] use semi-supervised machine learning to identify speech acts in email and forum posts. Ritter et al. [158] model Twitter conversations with Speech Act Theory in combination with topic modelling and show a Speech Act transition map with probabilities for each state.

One central use is in provenance in the Semantic Web. For assertions modelled in RDF, Carroll et al. [32] use the idea of performative warrants, to describe assertions made legitimately by the authority signing a Named Graph.

\subsection{Applications of the Language/Action Perspective}

Using the Language/Action Perspective and drawing from Speech Act Theory, Twitchell et al. [186] model online conversations to classify them and create visual maps, used for information retrieval:

"Using current search engines, the searcher could search for the words Vietnam, war, and critique. However, many critiques of the war might not contain the word critique, and would thus be lost (or receive a low ranking) in such a search. If the searcher was able to issue a query such as Vietnam war (critique) where critique is the purpose of at least one participant in the conversation, she would likely get better results. The search for the semantic meaning of the words Vietnam war using conventional searching techniques would then be combined with the search for the pragmatic force of the word critique, yielding a search result with higher precision than searching on semantic meaning alone." [186] (italic, underline added).

Attending to Speech Acts can also help predict deception, which uses 'fewer assertions and more expressives' [186].

\subsection{Applications of pragma-dialectics}

We are not aware of any argumentation tools specific to pragma-dialectics. de Moor, however, has taken a reconstructive approach to argumentation based on pragma-dialectics (e.g. [128]).

Pragma-dialectics has also been very influential in the argumentation community, integrated into e.g. Walton's textbook descriptions of argumentation [195] and discussed in at least one journal special issue [22] and edited collection [55].

\subsection{Applications of metadiscourse and structural elements of text}

Annotating argumentation in natural language often takes advantage of detecting metadiscourse and document structure. Two particularly promising approaches come from Teufel and Sándor. Teufel's rhetorical component extraction uses machine learning to extract and classify text according to its rhetorical status [182]. Sándor's concept-matching framework detects contrasting ideas linguistically, using metadiscourse and rhetorical markers [163]. Rather than determining the relations between text spans, Sándor uses her conceptmatching framework to infer contrasts, novel information, etc. from the author's metadiscourse [163].

Teufel and Moens focus on the document-level context, rather than the relationship between text spans. In their argument zoning, machine learning is used to extract and classify text from academic articles according to its rhetorical status [182]. Sándor and Teufel and Moens provide contributions in risk assessment, annotation, and audience- and task-specific summarization. Reuse of their work has included an application to find rhetorical features of related work sections, first using classification algorithms, and then applying ontologies [5]. However, these techniques are of particular interest because of further work in argument mining drawing on these ideas, which we soon discuss (Section 6.12.2, page 175).

\subsection{Applications of Rhetorical Structure Theory}

RST has been widely used for a variety of purposes and in 2006 a paper summarizing its applications [180] was published. Recently, Mentis et al. [123] used RST to analyze group decision rationale, comparing new and established groups using relations such as 'Interpretation \& Evaluation', 'Evidence', 'Elaboration', 'Concession', and 'Antithesis'. Summarization research has frequently drawn upon RST [120,121]. Some further applications of RST and related ap- 
proaches are discussed in a recent survey of work on discourse structure [200]; argumentation might be found in several genres they discuss, such as in the essay analysis and scoring and opinion mining applications.

\subsubsection{DILIGENT}

DILIGENT, briefly discussed above as an application of IBIS (Section 4.2, page 163), also draws from RST (Section 4.12, page 167) as shown in Fig. 8 on page 171 . To improve the agreement, clarity, and satisfaction [181] of discussions for ontology creation and refinement, DILIGENT restricts the argumentation. Five argumentative relations - alternative, evaluation and justification, counterExample, elaboration, and example - were drawn from RST [108], based on the arguments that advanced the ontology creation process in an experiment [141].

\subsubsection{Argumentation mining}

Drawing on rhetorical parsing, argument mining is a new area of study which seeks to detect and extract arguments from texts algorithmically. MochalesPaulau's dissertation work [124] focused on mining arguments [125,126,208] from the European Court of Human Rights and from the Araucaria annotated corpus $^{18}$, based on Context Free Grammars [208] as well as techniques from Teufel and Moens. Earlier Grover et al. [75] adapted Teufel and Moens' approach to determine the argumentative role of sentences drawn from a corpus of legal judgements.

In "Automatic Argumentation Detection and its Role in Law and the Semantic Web" [125], MochalesPaulau and Moens suggest that argument mining could contribute to the World Wide Argument Web [147], by extracting argument structures without human annotation. As they point out, automatic argument detection is needed at multiple levels: the inner structure of each argument as well as the overall structure of how multiple arguments are combined to contribute to the argumentative discourse.

\subsection{Applications of coherence}

Related notions of coherence are used in Thagard's explanatory coherence theory, a computational theory

\footnotetext{
${ }^{18}$ AraucariaDB: http://araucaria.computing.dundee.ac.uk/doku. php?id=argumentsources, the Arucaria corpus of arguments, draws in part from discussion fora (BBC Talking Point, Christian Apologetics \& Research Ministry Discussion Boards, MSNBC discussion forum, NPR discussion boards, and Global Greens) [95].
}

in which "an explanatory hypothesis is accepted if it coheres better overall than its competitors" [183]. This theory has been applied to analyze scientific reasoning and legal trials.

Another theory it influenced - that of cognitive coherence relations, which we discuss next - has been influential.

\subsection{Applications of Cognitive Coherence Relations}

Cognitive Coherence Relations contributed to the development of ScholOnto [118]. In separate work, Mancini's cinematic hypertext [117] used Cognitive Coherence Relations to develop a visual language for structuring hypertext links, increasing the coherence of argumentation conveyed in non-linear hypertext by clearly expressing the rhetorical relationships between chunks of text. Meanwhile, agent-based argumentation has used Cognitive Coherence Relations as a theory of pragmatics [136].

The ScholOnto [14,29] project, which ran at Open University's Knowledge Media Institute from 20012004, focused on modeling claims and arguments in scholarly communication. ClaiMapper, ClaiMaker, and ClaimSpotter were among the tools ${ }^{19}$ developed in the project, which was seen as part of sensemaking research. An open source web publishing tool called the Digital Document Discourse Environment ${ }^{20}$, or $D^{3} E$ [28] was also developed in related research. ScholOnto made an RDF Schema available, but database queries with SQL were preferred to querying based on this RDF Schema (SPARQL was first released as a working draft in 2004). The underlying ScholOnto ontology for these projects is shown in Fig. 12 on the next page. This ontology now underlies one of the applications we later discuss: Cohere is argument mapping software for sensemaking that integrates annotation and argumentation for the general public [26].

\section{Ontologies incorporating argumentation}

In addition to the seven ontologies discussed above, which implement or follow from particular theories ChAO, DILIGENT, IBIS RDF, SALT, ScholOnto, SIOC-Argumentation, and SWAN/SIOC - we now review seven ontologies relevant to argumentation. These include both dedicated argumentation ontolo-

\footnotetext{
${ }^{19}$ http://projects.kmi.open.ac.uk/scholonto/software.html

${ }^{20} \mathrm{http}: / / \mathrm{d} 3 \mathrm{e}$.sourceforge.net/
} 


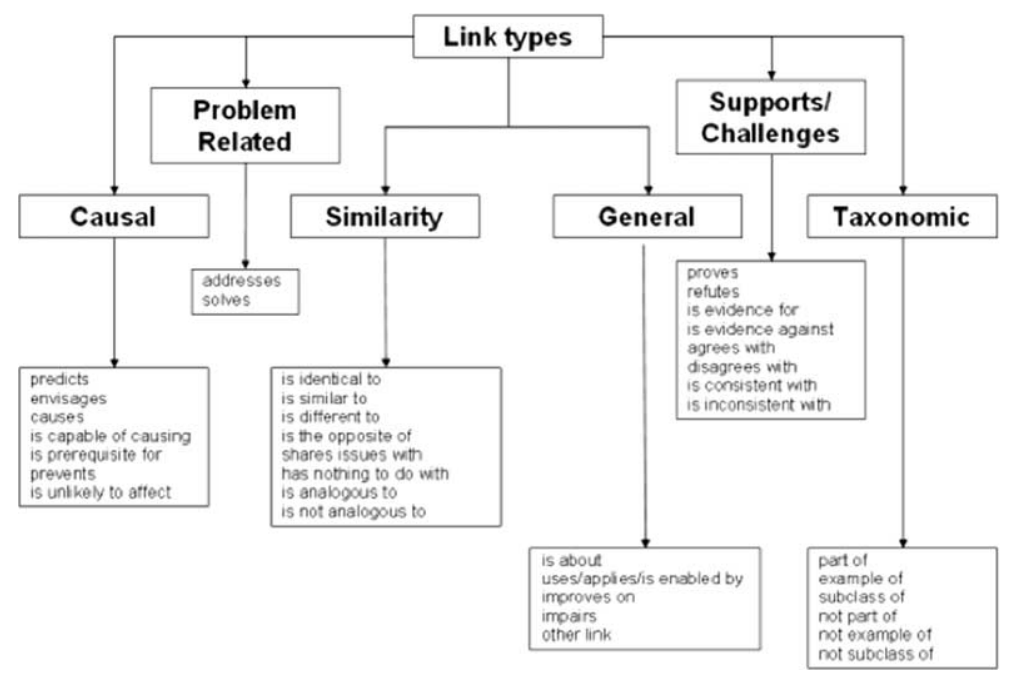

Fig. 12. Class structure of the Scholarly Discourse Ontology from [29].

gies (the Argument Interchange Format) and ontologies designed with substantial input from the argumentation community (the Legal Knowledge Interchange Format) as well as ontologies that incorporate small numbers of argumentative elements (the Annotation Ontology, bio-zen-plus, the Citation Typing Ontology, the Non-functional requirements and Design Rationale Ontology, and the Semantic Annotation Vocabulary).

\subsection{Argument Interchange Format (AIF)}

The Argument Interchange Format [41] is a powerful, dedicated ontology for argumentation, originally designed to ensure interoperability of argumentation software such as ArgDF, ArgKit, Carneades, and Online Visualisation of Argument. AIF would be challenging to apply to the Social Web because it requires argumentation schemes to be specified. In fact, even arguments themselves are not necessarily clearly specified in the informal argumentation found in the Social Web! Thus, for example, enthymemes make formal specification of arguments challenging [19].

The original core ontology, shown in Fig. 13 on the facing page consists of two disjoint sets of nodes: information nodes (I-nodes) holding the content of the argument and scheme nodes (S-nodes) holding the relationships between arguments. Scheme nodes are further divided into three main types, for representing logical inference (RA nodes), preferences or values (PA nodes), and conflicts between I-nodes (CA nodes). More recent work on AIF envisions classification of argumentation schemes, enabling automated reasoning over the schemes [145]. In Spring 2012, new specifications for AIF were released in OWL and RDF; an SQL database definition is also in progress ${ }^{21}$.

AIF forms the foundation for the World Wide Argument Web (WWAW). The WWAW is "a large-scale Web of interconnected arguments posted by individuals to express their opinions in a structured manner" [147], where RDFS and OWL were suggested to be used for AIF. The foundations of the World Wide Argument Web have been further discussed by Rahwan and others (e.g. [143,144,148]).

AIF has continued to develop, and several published extensions of AIF exist. Rahwan adds form nodes (F-nodes) [147] in order to more fully represent generic argument schemes (as opposed to the instantiations of those schemes). Then Walton's argument schemes can be represented, using ConflictSchemes to capture exceptions/Critical Questions. With AIF$\mathrm{RDF}^{22}$, Rahwan et al. [147] add RDFS extensions to an AIF implementation. In this implementation, edges are explicitly typed. Letia and Groza add a Context Node, used to evaluate the same argument in different contexts [109]. Rahwan et al. [145] present a new formalization of AIF in OWL-DL, implemented in Avicenna (Section A.9, page 200). Work on this area continues, largely published in the Computational Models of Argument (COMMA) conference series ${ }^{23}$, with a large body of promising research in the 2012 edition of this biannual conference.

\footnotetext{
${ }^{21}$ http://www.arg.dundee.ac.uk/?page_id=197

${ }^{22} \mathrm{http}$ ///argdf.org/source/ArgDF_Ontology.rdfs

${ }^{23} \mathrm{http} / / /$ www.comma-conf.org/
} 


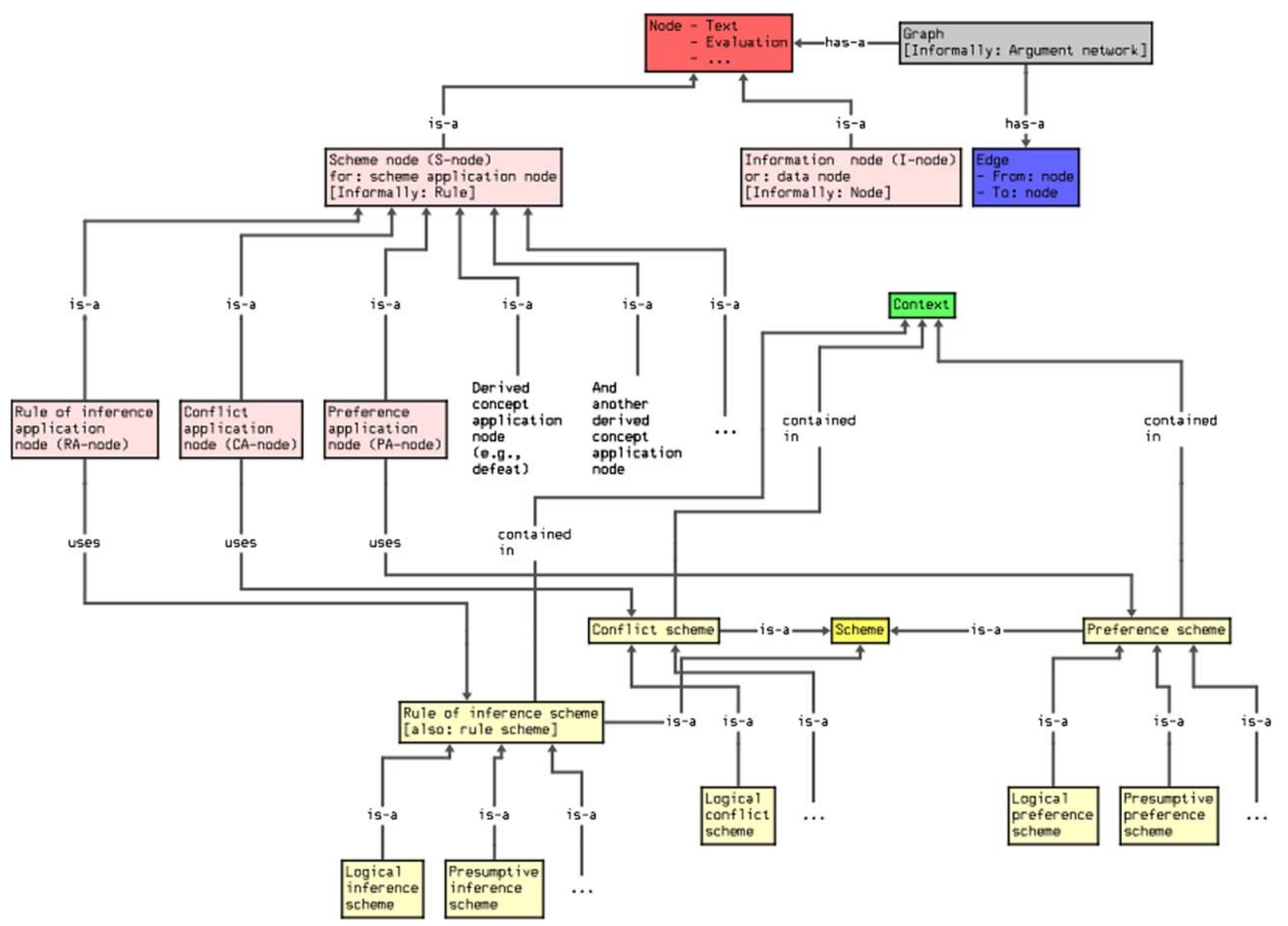

Fig. 13. Concepts and relations of the Argument Interchange Format.

While AIF was intended to model monological arguments, dialogue has been another area of interest in AIF extensions, with work from Modgil and McGinnis [127] and Reed et al. (e.g. [156]). Earlier work began the process of extending monological AIF for use in representing dialogical argumentation $[149,151,155,157]$.

\subsection{Annotation Ontology}

Argumentation enters into the Annotation Ontology's ${ }^{24}$ [45] curation use case. In that use case, a human curator reviews an annotation created by a text mining service, and first rejects it. This curator subsequently changes her mind after a discussion with a second curator, and finally accepts the annotation after all. The statuses Rejected, Discusses, and Accepted express an argumentative workflow in this situation.

\footnotetext{
${ }^{24}$ http://code.google.com/p/annotation-ontology/
}

The Annotation Ontology has been used by existing tools such as the SWAN Annotation Framework and Utopia PDF reader [10]. It is currently being incorporated into a new W3C standard for Open Annotation $^{25}$.

\subsection{Bio-zen-plus ontology framework}

The bio-zen-plus ontology $y^{26}$ [161] is an ontology for biology; as the name suggests, it is an extension of the bio-zen ontology ${ }^{27}$. It includes two argumentative properties, supported-by and in-conflictwith, augmenting the argumentation-related correlation-concepts, such as Positive correlation (unsigned), Positive correlation (signed), Negative correlation (unsigned), and Negative correlation

\footnotetext{
${ }^{25} \mathrm{http} / / /$ www.openannotation.org/spec/core/

${ }^{26} \mathrm{http}: / /$ neuroscientific.net/bio-zen-plus.owl

${ }^{27}$ http://neuroscientific.net/index.php?id=43
} 
(signed), which are found within the bio-zen ontology ${ }^{28}$.

\subsection{Citation Typing Ontology (CiTO)}

$\mathrm{CiTO}^{29}[171,172]$ is an ontology for citation networks in scholarly publications. Its argumentative terms include corrects, confirms, gives support to, is agreed with by, is ridiculed by, qualifies, and refutes. Papers can thus be semantically enhanced. ${ }^{30}$ For example, an author could indicate in a paper that it updates a previous publication, and critiques a piece of related work, while using evidence from another paper (citesAsEvidence). Readers can assemble bibliographies using CiTO properties, for instance with the bibliographic management software CiteULike ${ }^{31}$, showing the possibilities of semantic annotation.

\subsection{Legal Knowledge Interchange Format}

Legal Knowledge Interchange Format (LKIF) ${ }^{32}$, developed as part of the ESTRELLA project, is an OWL Ontology [133] for the legal domain. Its Rules \& Argumentation Module deals with Exceptions, Rules, Arguments, and Assumptions [62]. It also imports the LKIF Expression Module, which provides "a vocabulary for describing, propositions and propositional attitudes (belief, intention), qualifications, statements and media" [62]. It includes terms for various PropositionalAttitudes, as well as Intention and Lie, for instance.

\subsection{The NDR ontology}

The Non-functional requirements and Design Rationale (NDR) Ontology [111] addresses the visualization of non-functional requirements as Softgoal Interdependency Graphs. While some classes (such as Softgoal) are specific to this domain, the NDR Ontology introduces useful argumentative labels and causal relationships. For example, the label property can be used to indicate the extent to which goals are met (i.e. whether they are adequately 'satisficed'): Denied, Weakly denied, Undecided, Weakly satisficed, Satisficed, or Conflict.

\footnotetext{
${ }^{28}$ http://neuroscientific.net/bio-zen.owl

${ }^{29} \mathrm{http}: / /$ purl.org/spar/cito

${ }^{30}$ http://imageweb.zoo.ox.ac.uk/pub/2008/plospaper/latest/

${ }^{31} \mathrm{http}: / /$ www.citeulike.org/

${ }^{32} \mathrm{http} / / /$ www.estrellaproject.org/lkif-core/lkif-rules.owl\#
}

NDR also has classes for Argumentation, Claim, Contribution, and Interdependency (including a subclass, Correlation). The Contribution of child goals to the parent goal can be labelled as Break, Hurt, Unknown, Help, Make, etc.

\subsection{Semantic Annotation Vocabulary}

The Semantic Annotation Vocabulary [69] was developed for the Trellis system (Section A.35, page 212). They used various dimensions: pertinence, reliability, credibility, causality (e.g. contribute to, indicate), and temporal ordering, as well as structural relationships (such as part/whole, example-of, describes).

\section{Comparison of Semantic Web Models}

In Fig. 14 on the next page we present a comparison of the Semantic Web models discussed. Topics addressed include whether each ontology is centered on relations or concepts as well as whether it is IBISlike (i.e. does it contain concepts functionally equivalent to IBIS' 'Statement', 'Issue', 'Position', and 'Argument'?). We also cover what types of relations it contains, drawing from ScholOnto's types: causal, similarity, generic, supporting, challenging, taxonomic (e.g. hierarchical categorization), and problem related. Further, we describe whether polarity (e.g. positive vs. negative) and weights are explicit or implicit and whether the ontology specifies other ontologies to use for content provenance and authorship provenance (such as from FOAF, SIOC, or PAV - the Provenance $\&$ Authoring and Versioning ontology $\mathrm{y}^{33}$ ) and domain knowledge (such as from DOLCE, SKOS, or the PRotein Ontology). We have used a '?' to indicate that we were not able to find this information in publications, or when information was ambiguous, to reconcile it.

Some models provide a shallow view of arguments yet are situated within a larger (perhaps social) context. Yet other models, originating in the argumentation community, focus on representing the arguments themselves, often including the internal structure of the arguments. The argumentation community's interest in the Semantic Web has been motivated in part by the idea of The World Wide Argument Web (WWAW) [147], while the Semantic Web community's interest has centered on communication structures, rather than the details of argumentation or rhetoric.

\footnotetext{
${ }^{33} \mathrm{http}: / /$ swan.mindinformatics.org/spec/1.2/pav.html
} 


\begin{tabular}{|c|c|c|c|c|c|c|c|c|c|c|c|c|c|c|}
\hline & 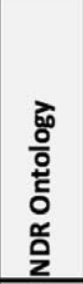 & $\begin{array}{l}\text { 峑 } \\
\underline{\underline{n}} \\
\underline{\underline{\omega}}\end{array}$ & 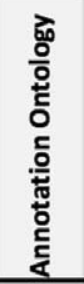 & $\begin{array}{l}\frac{n}{a} \\
\frac{n}{2} \\
\frac{0}{0}\end{array}$ & $\begin{array}{l}\sum_{\text {岀 }} \\
\text { 至 }\end{array}$ & 온 & 志 & $\begin{array}{l}\text { on } \\
\text { ঠั } \\
\text { 은 }\end{array}$ & 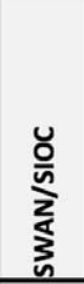 & 은 & 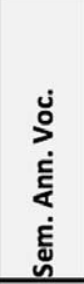 & $\begin{array}{l}\text { 온 } \\
\text { 응 } \\
\text { 돈 }\end{array}$ & 宸 & 宸 \\
\hline Concept/Relation centered & $\mathrm{R}$ & $\mathrm{C}$ & $\mathrm{R}$ & $\mathrm{R}$ & $\mathrm{R}$ ? & $\mathrm{R}$ & $\mathrm{C}$ & $\mathrm{C}$ & $\mathrm{R}$ & $\mathrm{R}$ & $\mathrm{R}$ & $\mathrm{R}$ & $\mathrm{C}$ & $?$ \\
\hline OWL/RDF & $\mathrm{OWL}$ & RDFS & OWL & OWL & OWL & RDFS & RDFS & RDFS & OWL & OWL & RDFS & RDFS & OWL & RDFS \\
\hline Statement & $\mathrm{N}$ & $\mathrm{Y}$ & $\mathrm{Y}$ & $\mathrm{N}$ & $\mathrm{Y}$ & $\mathrm{N} ?$ & $\mathrm{~N}$ & $\mathrm{Y}$ & $\mathrm{N}$ & $\mathrm{N}$ & $\mathrm{N}$ & $\mathrm{N}$ & $Y$ & $\mathrm{~N}$ \\
\hline Issue & $\mathrm{N}$ & $Y$ & $\mathrm{~N}$ & $\mathrm{~N}$ & $Y$ & $\mathrm{Y}$ & $\mathrm{N}$ & $Y$ & $\mathrm{~N}$ & $\mathrm{~N}$ & $\mathrm{~N}$ & $\mathrm{~N}$ & $\mathrm{~N}$ & $\mathrm{~N}$ \\
\hline Position & $\mathrm{N}$ & $\mathrm{Y}$ & $\mathrm{N}$ & $\mathrm{N}$ & $\mathrm{Y}$ & $\mathrm{Y}$ & $\mathrm{N}$ & $Y$ & $\mathrm{~N}$ & $\mathrm{~N}$ & $\mathrm{~N}$ & $\mathrm{~N}$ & $\mathrm{Y}$ & $\mathrm{N}$ \\
\hline Argument & $\mathrm{N}$ & $\mathrm{Y}$ & $\mathrm{N}$ & $\mathrm{N}$ & $\mathrm{Y}$ & $\mathrm{Y}$ & $\mathrm{Y}$ & $\mathrm{Y}$ & $\mathrm{N}$ & $\mathrm{N}$ & $\mathrm{N}$ & $\mathrm{N}$ & $\mathrm{N}$ & $\mathrm{N}$ \\
\hline Causal & $\mathrm{N}$ & $\mathrm{N}$ & $\mathrm{N}$ & $\mathrm{N}$ & $\mathrm{N}$ & $\mathrm{N}$ & $\mathrm{N}$ & $\mathrm{N}$ & $\mathrm{N}$ & $\mathrm{N}$ & $\mathrm{Y}$ & $\mathrm{Y}$ & $\mathrm{Y}$ & $\mathrm{N}$ \\
\hline Similarity & $\mathrm{N}$ & $\mathrm{N}$ & $\mathrm{N}$ & $\mathrm{N}$ & $\mathrm{N}$ & $\mathrm{N}$ & $\mathrm{N}$ & $\mathrm{N}$ & $\mathrm{N}$ & $\mathrm{N}$ & $\mathrm{N}$ & $Y$ & $\mathrm{~N}$ & $\mathrm{~N}$ \\
\hline Generic & $\mathrm{N}$ & $\mathrm{N}$ & $Y$ & $\mathrm{~N}$ & $Y$ & $Y$ & $\mathrm{~N}$ & $\mathrm{~N}$ & $Y$ & $Y$ & $\mathrm{~N}$ & $Y$ & $Y$ & $\mathrm{~N}$ \\
\hline Supporting & $Y$ & $\mathrm{~N}$ & $\mathrm{~N}$ & $Y$ & $\mathrm{Y}$ & $\mathrm{N}$ & $\mathrm{Y}$ & $Y$ & $\mathrm{Y}$ & $\mathrm{Y}$ & $Y$ & $\mathrm{Y}$ & $\mathrm{Y}$ & $\mathrm{Y}$ \\
\hline Challenging & $Y$ & $\mathrm{~N}$ & $\mathrm{~N}$ & $Y$ & $Y$ & $Y$ & $Y$ & $Y$ & $Y$ & $Y$ & $Y$ & $Y$ & $Y$ & $Y$ \\
\hline Taxonomic & $Y$ & $\mathrm{~N}$ & $\mathrm{~N}$ & $\mathrm{~N}$ & $\mathrm{~N}$ & $\mathrm{~N}$ & $\mathrm{~N}$ & $\mathrm{~N}$ & $\mathrm{~N}$ & $\mathrm{~N}$ & $\mathrm{~N}$ & $\mathrm{Y}$ & $\mathrm{Y}$ & $\mathrm{N}$ \\
\hline Problem-related & $\mathrm{Y}$ & $\mathrm{N}$ & $\mathrm{N}$ & $\mathrm{N}$ & $\mathrm{Y}$ & $Y$ & $Y$ & $\mathrm{Y}$ & $\mathrm{N}$ & $\mathrm{N}$ & $\mathrm{N}$ & $Y$ & $\mathrm{~N}$ & $\mathrm{~N}$ \\
\hline
\end{tabular}

(a)

\begin{tabular}{|c|c|c|c|c|c|c|}
\hline & Polarity & Weights & $\begin{array}{c}\text { Content } \\
\text { Provenance }\end{array}$ & $\begin{array}{l}\text { Authorship } \\
\text { Provenance }\end{array}$ & $\begin{array}{c}\text { Domain } \\
\text { knowledge }\end{array}$ & Design context \\
\hline NDR Ontology & Implicit & None & None & None & None & $\begin{array}{c}\text { Requirements } \\
\text { engineering }\end{array}$ \\
\hline IBIS RDF & Explicit & None & None & None & None & $\begin{array}{c}\text { General problem- } \\
\text { solving }\end{array}$ \\
\hline Annotation Ontology & Implicit & For correlations & FOAF, PAV & SIOC, PAV & Open & Annotation \\
\hline Bio-zen plus & Implicit & None & SIOC & FOAF & $\begin{array}{c}\text { DOLCE, SKOS, } \\
\text { Bio model }\end{array}$ & Biology \\
\hline DILIGENT & Implicit & None & ? & Actor name & None & $\begin{array}{c}\text { Collaborative } \\
\text { ontology design }\end{array}$ \\
\hline ChAO & Implicit & None & $\begin{array}{c}\text { System: author, } \\
\text { date, time }\end{array}$ & $\begin{array}{l}\text { System: author, } \\
\text { date, time }\end{array}$ & Open & $\begin{array}{c}\text { Collaborative } \\
\text { ontology design }\end{array}$ \\
\hline SALT & Implicit & None & Implicit & None & Open & $\begin{array}{c}\text { Scholarly } \\
\text { communication }\end{array}$ \\
\hline SIOC-Arg & Implicit & None & $\mathrm{SIOC}$ & $?$ & None & Social Web \\
\hline SWAN/SIOC & Implicit & Implicit & $\mathrm{SIOC}$ & $?$ & None & Scientific Discourse \\
\hline сіто & Implicit & None & None & None & None & Scholarly citations \\
\hline Sem. Ann. Voc. & Implicit & Implicit & $?$ & $?$ & None & Web of trust \\
\hline ScholOnto & Implicit & Implicit & None & None & None & Digital libraries \\
\hline LKIF & Explicit & None & Agents & $?$ & $?$ & Legal \\
\hline AIF & Explicit & None & $?$ & $?$ & $?$ & Argumentation \\
\hline
\end{tabular}

(b)

Fig. 14. A comparison of Semantic Web models for argumentation in terms of (a) structure; and (b) features.

\section{Features of Social Web tools}

We conducted a thorough review of argumentation tools for the Social Web, attending especially to existing Social Web sites, tools using the Semantic Web, and prototypes from the research community in Social Web and Semantic Web. In this section we describe re- lated reviews of argumentation tools, the scope of our review, and some highlights concerning thirteen particular features of these systems - visualization, ease of use, collaboration, user engagement, balancing contributions, deliberative polling, distributive and federated systems, annotation, incremental formalization, populating knowledge bases from user input, mixed ini- 
tiative, search, reasoning and querying. These provide ideas of the aspects that may be need to be considered for argumentation systems on the Social Semantic Web. For further detail about each system we reviewed, consult the Appendix, Section A, page 196.

\subsection{Related reviews}

Argumentation tools have been reviewed and overviewed in various publications, including two contemporary books. Visualizing Argumentation [96] presents eight chapters which cover the history and cognitive foundations of argumentation tools; describe tools for collaborative learning and deliberation; provide insight into map-based facilitation of in-person meetings; and describe mapping scholarly debates. Of particular interest in this exceptional volume is the chapter on "The Roots of Computer Supported Argument Visualization" [25]. Knowledge Cartography: Software Tools and Mapping Techniques [131] provides seventeen case studies of using mapping and argumentation tools, primarily in education, but also in science, politics, and organizational knowledge transfer.

Argumentation tools have also gained attention in e-government (e.g. [116], or for a tools review see 'Opinion gathering in e-democracy', Chapter 3 of Cartwright's dissertation, [34]) and education [164]. Crossover interest in politics from the IEEE community is evidenced by a 'Trends \& Controversies' section “AI, E-government, and Politics 2.0" [40].

Scheuer et al. [164] review 45 argumentation systems $^{34}$ used in Computer Supported Collaborative Learning and discuss 13 empirical studies involving the use of argumentation systems in education. Interesting results from their work are that arguments are constructed in learning applications in five main ways: free-form arguments, argumentation based on background materials, arguments rephrased (e.g. reworded and rekeyed) from a transcript, arguments extracted (e.g. copied/pasted) from a transcript, and systemprovided units, with combined approaches also used in some applications. Further, they compare the advantages and disadvantages of user-controlled and systemcontrolled layouts for education. Their discussion of ontologies is limited.

This tools coverage in this section differs from previous coverage in its scope of collaborative, Web-

\footnotetext{
${ }^{34} \mathrm{Six}$ of these systems are also discussed in our review below: Argunet, ConvinceMe, CoPe_it!, Debategraph, Debatepedia, and SEAS.
}

based tools with argumentation components, and in its attempt at comprehensiveness. A further bias has been software aimed at use by the public, rather than exclusively for government consultation, enterprise decision-making, or learning argumentation and critical thinking skills. However, we have deliberately included several research prototypes which focus on Semantic Web approaches to argumentation on the Web and on supporting the nascent World Wide Argumentation Web.

\subsection{Scope: Collaborative, Web-based tools with argumentation components}

Tools were considered in-scope if they were collaborative (i.e. involved sharing information among multiple parties who could build upon each others' work in some way), Web-based (i.e. allowed display of information on the Web), and had argumentative discussion components. By argumentative discussion, we mean discussion around disagreements, explanations, and reasons, coming from or including a rational (i.e. reason-based) standpoint.

Some prospective tools were excluded due to failing one or more of these conditions.

Tools failing the 'collaborative' criterion included the EUProfiler ${ }^{35}$, and the HealthCentral/Washington Post Poligraph $2008^{36}$. Users of these tools viewed personalized visualizations, based on their answers to a questionnaire, however they are not asked (or able) to share their comments on others' views, to interact with other users (adding to a larger debate), or to contribute to sensemaking or analysis of existing argumentation.

Tools failing the 'Web-based' criterion included email tools such as WIT and Zest, SAIC's SIAM and Causeway, and the argumentation tools Carneades, Araucaria, and Convince $\mathrm{Me}^{37}$. WIT ${ }^{38}$ [16] and Zest [210] focused on argumentation in email. SIAM ${ }^{39}$ and Causeway $^{40}$ are Windows-based software for influence net modeling, designed for analyst use and primarily for collaboration inside the firewall; although HTML can be exported, Web-based collaboration is not sup-

\footnotetext{
${ }^{35}$ http://euprofiler.eu/

${ }^{36} \mathrm{https}$ //www.washingtonpost.com/wp-srv/health/interactives/ poligraph/

${ }^{37}$ Note that we do include the similarly named 'ConvinceMe' site in Section A.17, page 204.

${ }^{38}$ http://www.w3.org/WIT/

${ }^{39} \mathrm{http}: / / \mathrm{www}$.inet.saic.com/inet-public/siam.htm

${ }^{40} \mathrm{http}$ ///www.inet.saic.com/inet-public/causeway.htm
} 


\begin{tabular}{|c|c|c|c|}
\hline $\begin{array}{l}\text { Argumentation } \\
\text { Design }\end{array}$ & Purpose & Orchestration & $\begin{array}{l}\text { Systemic } \\
\text { Rationality }\end{array}$ \\
\hline $\begin{array}{l}\text { Issue } \\
\text { networking } \\
\text { Examples: } \\
\text { - HyperNews } \\
\text { - QuestMzp } \\
\text { - Compendium }\end{array}$ & $\begin{array}{l}\text { A clash of claims } \\
\text { where the interaction } \\
\text { is organized into a web } \\
\text { of issues with relevant } \\
\text { positions and reasons } \\
\text { developed for } \\
\text { each issue. }\end{array}$ & $\begin{array}{l}\text { Enables a full exploration of the } \\
\text { agreements, disagreements, and } \\
\text { rationales for position but } \\
\text { typically provides no } \\
\text { functionalities for settling } \\
\text { differences. }\end{array}$ & $\begin{array}{l}\text { A situation calls for } \\
\text { participants to share, } \\
\text { explore, and learn about } \\
\text { each others' positions and } \\
\text { reasons and where these } \\
\text { will be held accountable } \\
\text { to the doubts of others. }\end{array}$ \\
\hline $\begin{array}{l}\text { Funneling } \\
\text { Examples: } \\
\text {-GroupSystems } \\
\text { - SAMM } \\
\text { - Other GDSS } \\
\text { - Voting tools }\end{array}$ & $\begin{array}{l}\text { Treat argumentation } \\
\text { as active consensus } \\
\text { formation where } \\
\text { interaction is } \\
\text { organized into a flow } \\
\text { from broad differences } \\
\text { toward an acceptable } \\
\text { conclusion. }\end{array}$ & $\begin{array}{l}\text { Sequences interaction into a } \\
\text { series of activities that } \\
\text { successively narrow a dispute or } \\
\text { decision toward the most } \\
\text { acceptable conclusion. The } \\
\text { functionalities such as } \\
\text { brainstorming, categorizing, and } \\
\text { voing provide means to remove } \\
\text { resistance to collective action. }\end{array}$ & $\begin{array}{l}\text { A situation calls for a } \\
\text { commitment by all } \\
\text { participants to a particular } \\
\text { course of action. This } \\
\text { stands in contrast to } \\
\text { issue-networking, which } \\
\text { aims primarily at } \\
\text { self-correction, } \\
\text { not at consensus }\end{array}$ \\
\hline $\begin{array}{l}\text { Reputation } \\
\text { Exomples: } \\
\text { - Experts-Exchange } \\
\text { - Sashdotcom } \\
\text { - Weblogs }\end{array}$ & $\begin{array}{l}\text { Create a knowledge } \\
\text { base for action by } \\
\text { pooling and refining } \\
\text { the expertise among } \\
\text { a community of } \\
\text { participants. }\end{array}$ & $\begin{array}{l}\text { Provides means like ratings for } \\
\text { individual participants to } \\
\text { compete with each other over } \\
\text { who provides the best answers } \\
\text { to the questions the community } \\
\text { most wants answered. The } \\
\text { orchestration produces experts } \\
\text { and a repository of answers. }\end{array}$ & $\begin{array}{l}\text { A situation calls for } \\
\text { discovering and cuitivating } \\
\text { trustworthy experts. It } \\
\text { stands in contrast to both } \\
\text { funneling, because it does } \\
\text { not aim at consensus, and } \\
\text { issue-networking because } \\
\text { people are not separated } \\
\text { from their standpoints. }\end{array}$ \\
\hline
\end{tabular}

Fig. 15. Issue-networking, funnelling, and reputation, from [128].

ported. Similarly, Carneades ${ }^{41}$ maps can be shared in LKIF, but not directly visualized online. Araucaria ${ }^{42}$ $[152,154,160]$ offers a searchable online argument corpus, but not online display of its arguments. While Convince $\mathrm{Me}^{43}$ offers a Java applet for display, arguments cannot be saved or published via the applet.

Tools failing the 'argumentative discussion' criterion included general Web2.0 tools as well as Anekdotz, and Vox Populi. General Web2.0 tools (e.g. Twit$\operatorname{ter}^{44}$, Facebook ${ }^{45}$ ) and social software (generic mailing lists, forums) were excluded since their argumentation support is peripheral. Anekdotz ${ }^{46}$ failed because the sites currently using it focus on the emotional, rather than the rational, aspects of argumentation. For example, the breakups section of When You Knew asks commenters to click on either 'Put their stuff on the curb' or 'Give em another shot' to solicit feedback, which is marked as positive, negative, or neutral. Vox Populi ${ }^{47}$ [21] supports documentary filmmakers in generating argumentative film sequences based on annotated interviews.

Further, tools were treated differently depending on their origin and availability; for instance, it was considered helpful to include many contemporary research

\footnotetext{
${ }^{41} \mathrm{http}: / /$ carneades.berlios.de/

${ }^{42} \mathrm{http}$ ///araucaria.computing.dundee.ac.uk/

${ }^{43} \mathrm{http}: / /$ codeguild.com/convinceme/

${ }^{44}$ http://twitter.com

${ }^{45}$ http://www.facebook.com/

${ }^{46} \mathrm{http}: / /$ www.anekdotz.com/

${ }^{47}$ http://homepages.cwi.nl/ media/demo/IWA/
}

systems even though we were not able to interactively explore Web-based demo versions for some of those systems. We have inevitably missed some relevant systems, and would appreciate the reader's assistance in fixing this flaw.

\subsection{Classifications of social tools}

Aakhus and collaborators $[1,128]$ classify argumentation software by use: issue networking, funnelling, or reputation (Fig. 15). Shum says that each tool is 'tuned' to a different task: "foraging for material, classifying and linking it, discussing it in meetings and online, and evaluating specific points in more depth" [27]. We later use this categorization, as 'Functional type' in our comparison of tools.

Scheuer et al. [164] compare the visualization and representation styles of argumentation tools used in computer-supported collaborative learning. They summarize the pros and cons of five representation styles, as shown in Fig. 16 on the next page. We later use this categorization, as 'Representation style' in our comparison of tools. Scheuer's representation styles are typically used for discussions (linear representation), modeling (container ${ }^{48}$ ), or both (threaded, graph). For instance, graph representations are highly expressive, with explicit labelling of relationships, but make it hard to see temporal sequences.

\footnotetext{
${ }^{48}$ The container approach uses discrete visual areas to group related items. For example in Debatepedia each question is contained in a frame with pro and con arguments on that question.
} 


\begin{tabular}{|c|c|c|c|}
\hline Representation style & Typical uses & Pros & Cons \\
\hline Linear (e.g., chat) & $\begin{array}{l}\text { - Discussions } \\
\text { (especially } \\
\text { synchronous) }\end{array}$ & $\begin{array}{l}\text { - Familiar and intuitive to most } \\
\text { users, easiest to use } \\
\text { - Best to see temporal sequence } \\
\text { and most recent contributions }\end{array}$ & $\begin{array}{l}\text { - Risk of sequential incoherence } \\
\text { (McAlister et al. 2004) } \\
\text { - Not suited to represent the } \\
\text { conceptual structure of } \\
\text { arguments } \\
\text { - Lack of overview }\end{array}$ \\
\hline $\begin{array}{l}\text { Threaded (e.g., forums, } \\
\text { Academic Talk) }\end{array}$ & $\begin{array}{l}\text { - Discussions } \\
\text { (especially } \\
\text { asynchronous) } \\
\text { - Modeling }\end{array}$ & $\begin{array}{l}\text { - Familiar and intuitive to most } \\
\text { users, easy to use } \\
\text { - Easy to manage large } \\
\text { discussions } \\
\text { - Addresses issue of sequential } \\
\text { incoherence }\end{array}$ & $\begin{array}{l}\text { - Moderately hard to see temporal } \\
\text { sequence (because of multiple } \\
\text { threads) as compared to Linear } \\
\text { - Limited expressiveness (only } \\
\text { tree-like structures) }\end{array}$ \\
\hline $\begin{array}{l}\text { Graph (e.g., Belvedere, } \\
\text { Digalo) }\end{array}$ & $\begin{array}{l}\text { - Discussions } \\
\text { - Modeling }\end{array}$ & $\begin{array}{l}\text { - Intuitive form of knowledge } \\
\text { modeling (Suthers et al. 1995) } \\
\text { - Highly expressive } \\
\text { (e.g., explicit relations) } \\
\text { - Many graph-based modeling } \\
\text { languages exist }\end{array}$ & $\begin{array}{l}\text { - Hard to see temporal } \\
\text { sequence } \\
\text { - Lack of overview in large } \\
\text { argumentation maps (need a } \\
\text { lot of space, can lead to } \\
\text { "spaghetti" images (Hair } \\
\text { 1991; Loui et al. 1997) }\end{array}$ \\
\hline $\begin{array}{l}\text { Container (e.g., } \\
\text { SenseMaker, Room 5) }\end{array}$ & - Modeling & $\begin{array}{l}\text { - Easy to see which argument } \\
\text { components belong together } \\
\text { and are related }\end{array}$ & $\begin{array}{l}\text { - Limited expressiveness (e.g., } \\
\text { only implicit relations, only } \\
\text { tree-like structures) } \\
\text { - Lack of overview in large } \\
\text { argumentation maps because } \\
\text { of missing relations }\end{array}$ \\
\hline Matrix (e.g., Belvedere) & - Modeling & $\begin{array}{l}\text { - Easy systematic investigation } \\
\text { of relations } \\
\text { - Missing relations between } \\
\text { elements are easily seen } \\
\text { (Suthers 2003) }\end{array}$ & $\begin{array}{l}\text { - Limited expressiveness (e.g., } \\
\text { supports only two element } \\
\text { types (row, column), no } \\
\text { relations between relations) } \\
\text { - Uncommon (Non-intuitive) } \\
\text { way of making arguments }\end{array}$ \\
\hline
\end{tabular}

Fig. 16. Comparison of the visualization and representation styles of CSCL argumentation tools, from [164].

\subsection{List of social and Semantic Web tools considered}

In this section we draw from our review of thirtyseven online argumentation tools: AGORA: Participate - Deliberate, ArgDF, Arguehow, Argument Blogging, Argumentum, Argumentations.com, Argunet, Avicenna, bCisiveOnline, Belvedere, Cabanac's annotation system, Climate CoLab, Cohere, Competing Hypotheses, ConsiderIt, ConvinceMe, CoPe_it!, CreateDebate, Debate.org, Debategraph, Debatepedia, Debatewise, DiscourseDB, Dispute Finder, Hypernews, LivingVote, Opinion Space, Online Visualisation of Arguments, Parmenides, PDOnline, REASON, Riled Up!, SEAS, Trellis, TruthMapping, and Videolyzer. These systems are described and discussed individually, in alphabetical order, in the Appendix, Section A, page 196.

\subsection{Visualization}

Overviews and visual representations aid understanding. Here we point out visualization features of some Social Web argumentation tools, along with screenshots; further details are available in the appendix. Argument maps are one classic representation, which continues to be popular with a variety of tools, including Argunet, Cohere, and Climate CoLab.

On Argunet, users have significant control over the presentation of arguments, such as colors and descriptions of different argument families. Related maps can be published in series, as shown in Fig. 17(a) on page 183. In the argument map representation, each node can be opened up to reveal a matrix listing which other arguments support, attack, are supported by, and are attacked by the given node (Fig. 17(b) on page 183).

In Argumentum ${ }^{49}$ arguments are colored to indicate the supporting (green) and opposing (red) arguments (Fig. 18 on the facing page). Comments, but not their replies, are similarly colored to indicate agreement or disagreement. Pro and con arguments are distinguished by green and red lines to the left of a comment, posted linearly, rather than in two columns.

\footnotetext{
${ }^{49}$ http://arg.umentum.com/
} 


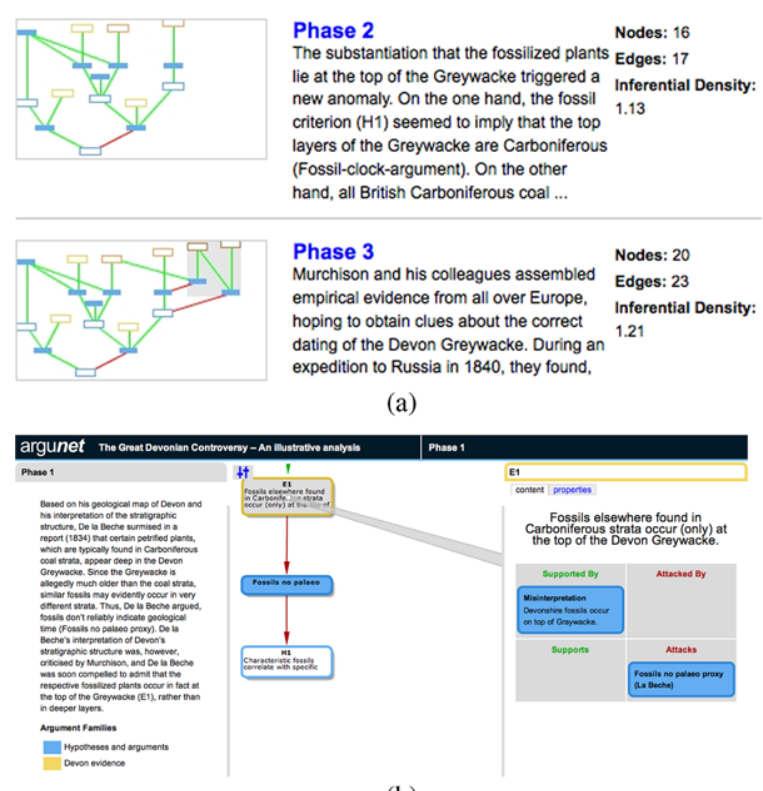

(b)

Fig. 17. Argunet can show an (a) overview of several related argument maps; and (b) in each individual map, nodes can be opened up to show arguments they support, attack, are supported by, and are attacked by. (Color figure online)

Competing Hypotheses ${ }^{50}$ supports breaking down information into hypotheses, evidence, and analysis, which are entered into a matrix as shown in Fig. 19(a) on page 184 . The matrix can help visually indicate the most likely and least likely scenarios. ${ }^{51}$ Multiple analyses can be combined to provide a group view (Fig. 19(b) on page 184), or compared pairwise.

ConsiderIt $^{52}[102,103]$ powers the Living Voters' Guide $^{53}$. What is unique is the possibility to drill down to understand other voters' perspectives. In addition to seeing pros and cons on an issue from all voters, regardless of their stance, (Fig. 20(a) on page 184), the Living Voters' Guide can show the key points for a particular group of voters (Fig 20(b) on page 184), such as those undecided on the issue or strongly supporting it. This can help users understand what makes an issue controversial. Users indicate how they feel about an issue before and after reading an argument (deliberative polling), which could also be used to find the most convincing arguments.

\footnotetext{
${ }^{50}$ http://competinghypotheses.org/

${ }^{51}$ More sophisticated ACH-based software uses matrices as input to Bayesian probabilistic reasoning.

${ }^{52} \mathrm{http}$ ///engage.cs.washington.edu/considerit/

${ }^{53} \mathrm{http} / / / \mathrm{www}$. livingvotersguide.org/
}

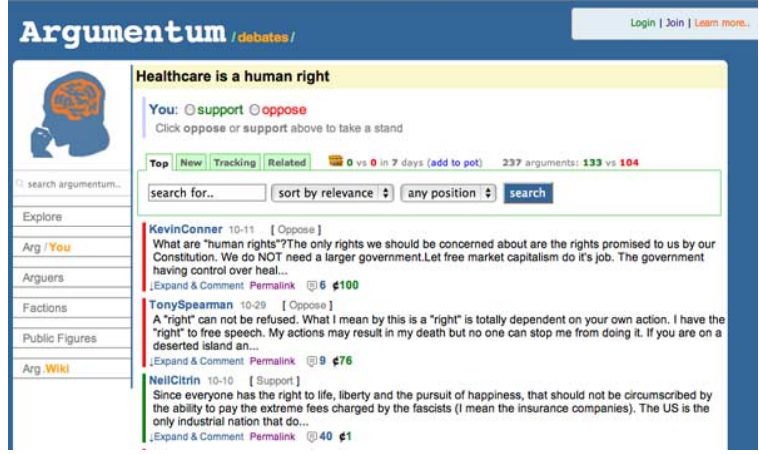

Fig. 18. In Argumentum, the left-hand color bars indicate the supporting (green) and opposing (red) arguments. (Color figure online)

CreateDebate (Fig. 21 on page 185) offers numerous statistics for each debate, such as the language grade level, average word lengths, and vocabulary overlap, as well as a wordcloud. Some debates have more than two sides. Replies can 'support', 'dispute', or 'clarify' a given point.

In Opinion Space [63], opinions are mapped in a constellation, using principal component analysis, to show a user where they stand compared to other respondents, as shown in Fig. 22 on page 185. Each point in the visualization represents a perspective; larger points represent more popular perspectives.

SEAS [113,114] structures arguments as templates, showing a colored tree view (Fig. 23 on page 185). SEAS visualization features are also considerable: to visualize multiple dimensions, SEAS uses starburst, constellation, and table views.

\subsection{Ease of use}

By 'ease of use', we mean how easy an interface is to use, based on our own perception. ConvinceMe $e^{54}$ lets users add arguments to pro or con columns, or add a rebuttal by clicking a button. Arguments can be voted up or down. Various other systems (discussed in the appendix) have similar functionality.

Debatepedia ${ }^{55}$, a wiki-based system, provides an intuitive editing environment, where users can edit the entire page or just the relevant section, such as the pro or con for a topic.

Living Vote ${ }^{56}$ asks participants to vote on arguments in a tree. "To vote on an argument, you must first prove you've read it by answering a simple question." Users can also add arguments to the tree.

\footnotetext{
${ }^{54} \mathrm{http} / / / \mathrm{www} . \mathrm{convinceme.net/}$

${ }^{55} \mathrm{http}: / /$ debatepedia.idebate.org/

${ }^{56}$ http://www.Living Vote.org/
} 


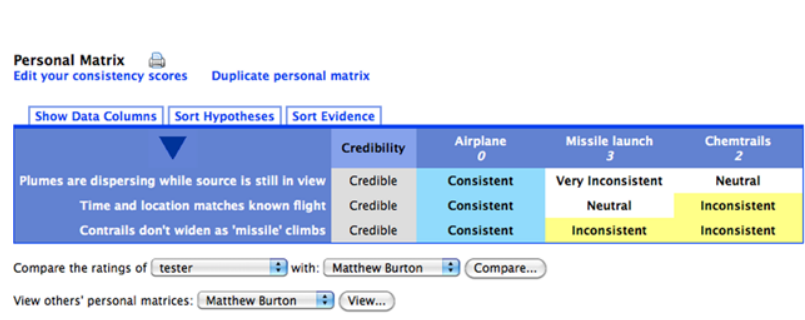

(a)

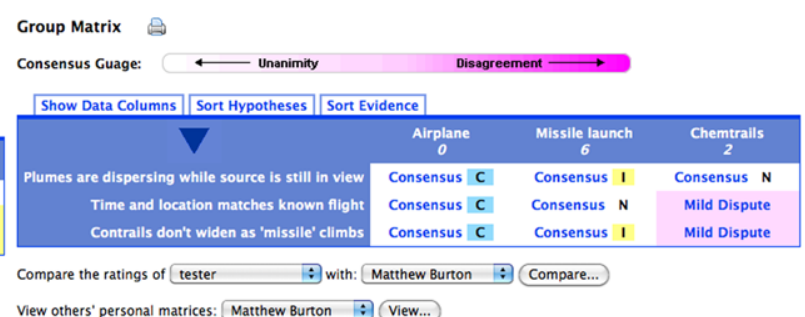

(b)

Fig. 19. In Competing Hypotheses, (a) each individual's analysis is represented in a consistency matrix; (b) multiple analyses can be combined to create a group matrix. In the group view, darker shades of purple indicate more disagreement. (Color figure online)

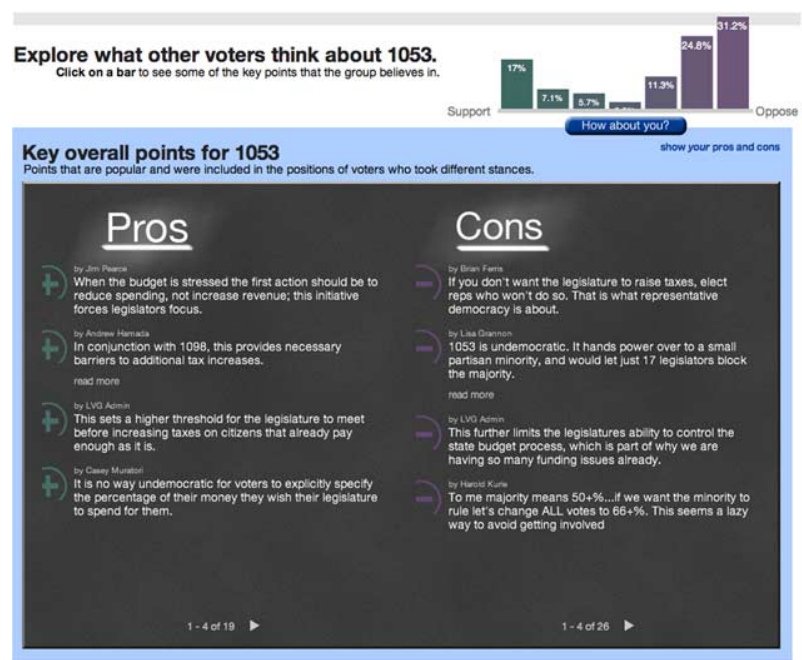

(a)

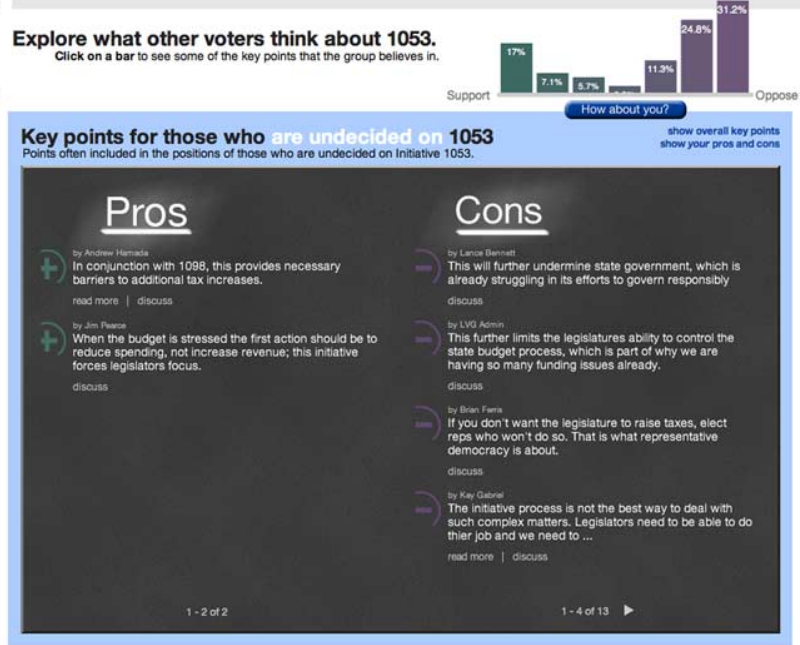

(b)

Fig. 20. The Living Voters' Guide compiles pro and con lists on each issue. They give (a) an overview of what all voters think about the issue; as well as (b) the key points for undecided voters. (Color figure online)

\subsection{Collaboration}

Systems approach collaboration in various ways.

bCisive Online is intended for real-time collaboration in conjunction with with audio conferencing. One person edits the map at a time, adding nodes and connections between nodes while others can point with their cursor or request editing control.

Climate CoLab uses moderation to help review comments and deal with the learning curve for argument mapping. While any user can add Pros, Cons, and Issues directly to an argument map, moderators are expected to keep track of the conversation, adding new ideas to the argument map as needed.

Competing Hypotheses ${ }^{57}$ has persistent chat (essentially a comment thread) for the entire project as well

\footnotetext{
${ }^{57}$ http://competinghypotheses.org/
}

as message boards for each hypothesis, evidence item, and evidence-hypothesis pair.

On Debatewise ${ }^{58}$, everyone can collaborate in creating the strongest case both for and against a given issue, using teams.

\subsection{User engagement}

Debatewise also makes it easy to get involved by providing suggestions of 5-minute, 20-minute and 1-hour tasks and showing "7 things you should have an opinion on" in rotating images on the homepage.

Social networking is one part of the engagement at Debate.org, which allows users to search for people with particular profile attributes, such as income, location, ideology, gender, president, religion, and who they are interested in and looking for.

\footnotetext{
${ }^{58} \mathrm{http}: / /$ debatewise.org/
} 


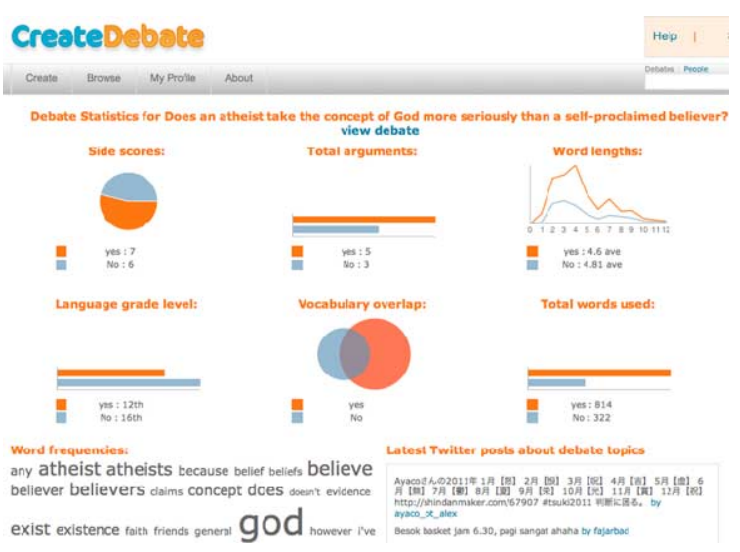

Fig. 21. At CreateDebate, users add and comment on pro and con arguments. (Color figure online)

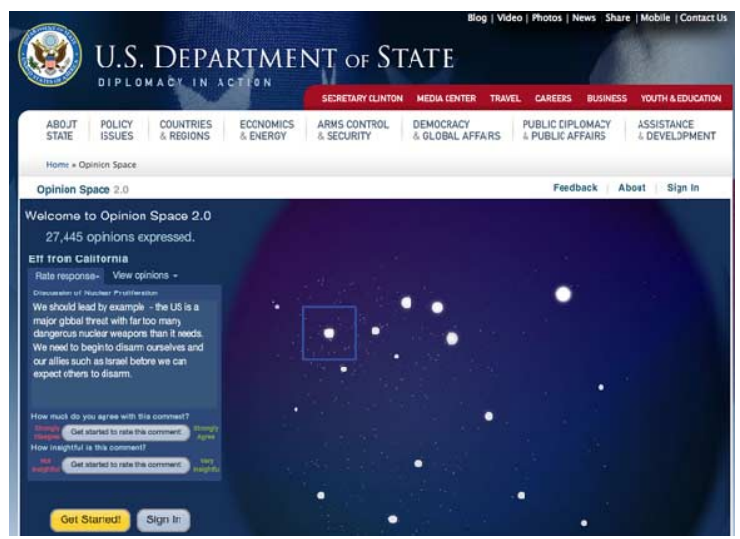

Fig. 22. Opinion Space maps comments in a constellation view. (Color figure online)

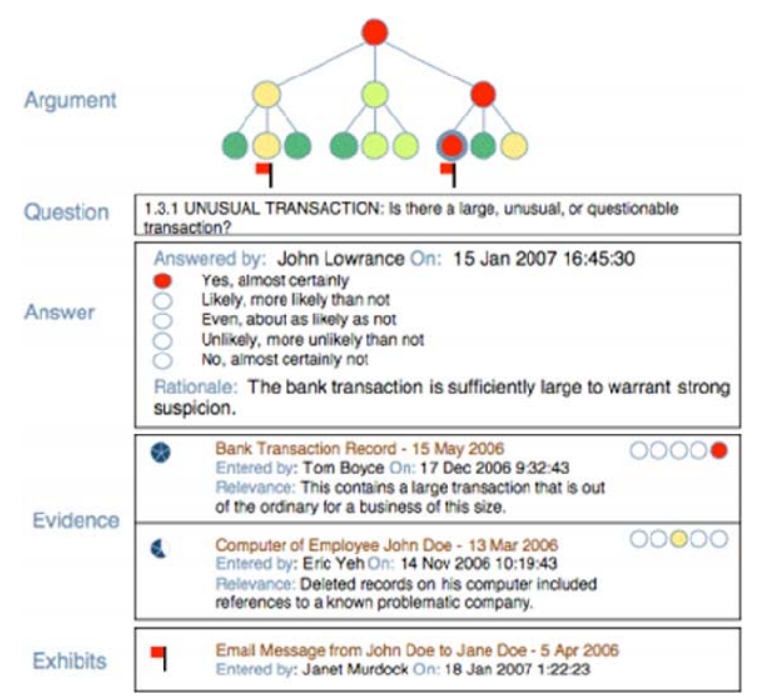

Fig. 23. SEAS visualizes arguments as a tree; colors indicate the credibility level [113]. (Color figure online)
Along with adding comments, on many sites, users can vote for arguments that convinced them (e.g. Create Debate). A user's reputation is generally based on the success of their arguments. Votes may be weighted, for instance after 10 up or down votes, further votes have less influence on ArgueHow.

Competition helps engage users at other sites, which can be quite explicit about this aspect; for instance, Riled Up!'s tagline is "Like Raising Cain? So Do We." Other sites, like ConvinceMe treats debates as games; in one such game, the debater whose idea is most popular is crowned "King of the Hill". Competitive debating environments may use point schemes and user rankings to motivate contributions.

\subsection{Balancing contributions}

There are several approaches to balancing contributions. For example, by removing authorship markers, argument maps may increase the neutrality of a conversation.

Another approach to balancing is taken by TruthMapping ${ }^{59}$, which focuses on having a persistent conversation which can not be drowned out by a single opponent. Users can leave feedback in critiques attached to each premise and conclusion (Fig. 53(b) on page 213), users can continually modify each contribution, but can only post one critique on each node. Anyone can leave a rebuttal, but only one user, the original arguer, can modify the map. The system indicates when comments are out of sync with the map, and a wiki-style history is available to better trace the conversation.

\subsection{Deliberative polling}

One measure of arguments is how persuasive they are. Measuring how users feel about an issue before and after reading an argument - known as deliberative polling [65] - is used in some systems, such as ConsiderIt, Living Vote, and OpinionSpace.

Debate.org uses deliberative polling but places more importance on other factors besides agreement when scoring debates, giving the most importance to using reliable sources and making convincing arguments.

\subsection{Distributed and federated systems}

Systems can be distributed, like Argument Blogging, in which JavaScript code is placed on blogs to link

\footnotetext{
${ }^{59}$ http://www.truthmapping.com/
} 
back to a server which provides access to a larger conversation.

Federation is an exciting direction: Argunet [166] uses an open source federation system for sharing argument maps from a desktop tool ${ }^{60}$. Uses can run their own server or use a public server, Argunet.org ${ }^{61}$, which allows authors to make maps public or restrict viewing and/or editing to a specified group.

These mechanisms also impact the privacy of a system - whether work can be saved privately, used collaboratively with a small group, or shared publicly with the world.

\subsection{Annotation}

Annotating materials or commenting on existing discussions can be an important way to interact on the Social Web. Annotation can also be used to classify messages. For instance, Hypernews ${ }^{62}$ [18] asks users to indicate what kind of message they are posting (None, Question, Note, Warning, Feedback, Idea, More, News, Ok, Sad, Angry, Agree, Disagree).

Videolyzer $^{63}$ [51] provides an integrated discussion forum for annotating and challenging the claims a video makes. Segments likely to be of interest are identified ahead of time by processing both the transcript and the video.

Annotation has also been treated in from an argumentation perspective in the research of Cabanac et al., who study social validation of argumentative debates through collective annotations [30].

\subsection{Incremental formalization}

With incremental formalization, representations are useful before they are fully interpretable by the computer. Incremental formalization can be helpful since people find it difficult to make structure, content, or procedures explicit [170]. As the user's understanding (or goals) change, some systems facilitate systematic additions or changes.

With Argunet, arguments can be quickly sketched or reconstructed as premises and conclusions, supporting incremental formalization.

CoPe_it! transforms the user-created informal spatial hypertext view (Fig. 39(a) on page 205) into an is-

\footnotetext{
${ }^{60} \mathrm{http}: / / \mathrm{www}$. argunet.org/editor/

${ }^{61} \mathrm{http}: / /$ www.argunet.org/debates/

${ }^{62} \mathrm{http}: / / \mathrm{www} . h y p e r n e w s . o r g / H y p e r N e w s / g e t / h y p e r n e w s / r e a d i n g$. html

${ }^{63} \mathrm{http} / / /$ videolyzer.com/
}

sue chart (Fig. 39(b) on page 205) according to rules shown in Fig. 39(c) on page 205. Users can also customize the transformation rules.

\subsection{Populating argumentative knowledge bases from user input}

Other approaches are also iterative, similar to incremental formalization but focusing specifically on the input phase. For instance, user input may be processed, and the output presented to the user, who can then correct it.

Trellis introduced a language processing technique called "Annotation Canonicalization through Expression synthesis" [20], which applied an ontology to a user-supplied sentence, checked the computer's ontology application by presenting a paraphrase to the user, and solicited additions to the ontology from unknown or misunderstood words.

Controlled natural languages, which adopt a more restrictive grammar and vocabulary in order to facilitate parsing, have also been used to take in information, formalizing it for reasoning. For instance, Wyner et al. [207] propose using a controlled natural language called Attempto Controlled English [48] for high-stakes argumentative discussions, in order to generate a first-order-logic representation of the discussion.

Dispute Finder ${ }^{64}[60,61]$ provides just-in-time information, alerting users when information they read is disputed, based on its database of disputed claims. This relies on a disputes database which was first populated by hand-annotation by activists (interested in informing or convincing others) and then extended algorithmically. The algorithm, which can be applied on the Web at large, uses a set of 54 patterns to identify possible disputed claims.

\subsection{Mixed initiative}

Another possibility is to use Mixed Initiative systems, wherein the actions of both humans and machines are important. Online Visualisation of Argument $(O V A)^{65}$ is part of a pipeline of argumentation tools [174] which starts to bridge the gap between human-oriented argumentation tools and calculationbased agent argumentation. Mixed initiative discussions are enabled by the argument maps created by

\footnotetext{
${ }^{64}$ http://ennals.org/rob/disputefinder.html

${ }^{65} \mathrm{http}$ ///ova.computing.dundee.ac.uk
} 
OVA or any other AIF-based tool. Thus, instead of representing one's point of view countless times in a forum or FAQ, it would be possible to delegate these conversations to a machine agent using an underlying argument map, as prototypes like MAgtALO ${ }^{66}[155,202]$ and the Google Wave discussion bot Arvina show.

\subsection{Search}

Semantic search focuses not on mere keyword matches but on retrieving structured data, such as whether an opinion is argued for or against. Semantic search is possible with several tools. $\operatorname{ArgDF}{ }^{67}$ uses the AIF-RDF ontology described above [147,148,212] and Sesame RDF ${ }^{68}$. In ArgDF, it is possible to display all the arguments in which a claim is involved (e.g. where it is used as a conclusion or as a premise) or all the arguments using, say, the Argument from Expert Opinion reasoning scheme.

Discourse $D B^{69}$ uses Semantic MediaWiki [104] with the SemanticForms ${ }^{70}$ extension. This makes it possible to list all commentary written by particular person, published in a particular venue, and so forth. Further, since items indicate the position they take on a topic, DiscourseDB can list all commentary for or against a given position. When a topic has multiple positions (e.g. Darfur ${ }^{71}$ ), DiscourseDB is especially helpful in summarizing the discussion. Semantic search uses a simple syntax: for instance, on the Darfur conflict issue, to search for commentary opposing the position that the U.N. should send peacekeepers, this code is used: [[is against::Darfur conflict/United Nations should send peacekeepers]]. Since the results are already displayed on summary pages, most end users would not need to create or modify queries, but DiscourseDB's semantic search is a powerful tool for creating summaries.

\subsection{Reasoning and querying}

As previously mentioned, Attempto Controlled English can generate first-order-logic representations, which allow inference and consistency-checking, and can be translated into OWL and RuleML [66]. Mean-

\footnotetext{
${ }^{66} \mathrm{http}: / /$ www.arg.dundee.ac.uk/?page_id=61

${ }^{67} \mathrm{http}: / / \operatorname{argdf}$. org/

${ }^{68}$ Various other tools tools export AIF without directly implementing semantic search.

${ }^{69} \mathrm{http}: / /$ discoursedb.org/

${ }^{70} \mathrm{http}: / /$ www.mediawiki.org/wiki/Extension:Semantic_Forms

${ }^{71}$ http://discoursedb.org/wiki/Darfur_conflict
}

while, Open Vocabulary Executable English can be used for simple reasoning in the Internet Business Logic System ${ }^{72}$.

Parmenides $^{73}$ [35-37] is a structured survey tool for gathering public opinion on a proposal. Based on argument schemes and critical questions from argumentation theory, Parmenides can pinpoint the source of the disagreement, by having participants respond to a series of questions. At the end of the survey, users are offered the choice of submitting an alternative proposal, and are shown the answers they chose. Administrators can then analyze the group's responses, which are displayed in graphical argumentation frameworks [52].

More advanced reasoning and querying is enabled by Avicenna, an OWL-based argumentation system on the Web which uses Jena [33], $\mathrm{ARQ}^{74}$, and Pellet [173]. Since OWL supports inference over transitive properties, Avicenna can support argument chaining, such as retrieving all arguments that directly or indirectly support a given conclusion. Avicenna is also used to infer the classification hierarchy of argument schemes: for example, an appeal to expert opinion is a specialization of an argument from position to know.

\section{Discussion \& conclusion}

We have reviewed argumentation theory, existing ontologies with argumentative aspects, and Web tools for argumentation. We now discuss three main gaps based on our observations. First, the ontologies given need further adaptation to meet the existing variety of social tools and purposes. In particular, arguing is a social activity. The varieties of argument tools on the Social Web need distinct types of interface support and social engineering. The remaining question is what appropriate Social Web ontologies for argumentation would be. Rather than a single ontology, we envision modular, interoperable components.

\subsection{Arguing is a social activity}

As argumentation scholars have long realized, humans argue for a variety of reasons, not always to solve "wicked problems". Rather, arguing is a social activ-

\footnotetext{
${ }^{72} \mathrm{http}: / /$ www.reengineeringllc.com/

${ }^{73} \mathrm{http}$ ://cgi.csc.liv.ac.uk/ parmenides/

${ }^{74} \mathrm{http}: / /$ jena.sourceforge.net/ARQ/
} 
ity people may use to position and establish themselves. This kind of arguing is important in the Social Web, where people play by arguing such as with ConvinceMe's the 'King of the Hill' game, or create networks of friends and enemies, such as on Riled Up! and Create Debate. Arguing can also be used to connect people such as on Debate.org. Ontologies for the Social Semantic Web will need to respect these social aspects, and may need to incorporate emotive indicators such as the heat of the debate as well as the manner in which the outcome will affect the participants.

The notion of debate, where two parties face off, is also well-represented in existing social tools. Debate may allow individuals to show their expertise, to find the best arguments, or simply to practice their rhetorical skills. Debate topics may be reused, for ongoing issues with two or more defendable positions, especially when a topic is controversial. This suggests two opportunities. First of all, future Social Semantic Web prototype tools for sensemaking and argument mapping could be tested for argumentation for some common debate topic in order to find a large audience of potential evaluators. Second, providing meaningful ways to discover new debate topics, and potentially record and share the outcome of these debates, could be helpful. Frequent debaters may also provide an interesting class of users since we might expect them to be more familiar with fallacies and argument diagramming, making them potentially more savvy about argumentation schemes and similar abstractions.

\subsection{Current use}

While argumentation support has become more mainstream, it is still a niche. While there is a desire for public discussion systems, especially in areas such as e-government, social discussion systems and social networks are driven by network effects (e.g. you are persuaded to use them by the ability to communicate with your friends and colleagues) and by ease of use. Argumentative elements in generic social media tools are very basic: Facebook and Google Plus use 'Like' and ' +1 ' buttons, which imply a semantics of agreement; YouTube adds a 'dislike' button, and flagging posts for moderation (e.g. on Craigslist) or downvoting posts (e.g. on StackOverflow or Reddit) also implies dislike.

With existing systems, discussed in the Appendix, there is a continuum from those with little use to those with wide use. Some (non-research) sites have few users and seem to have been abandoned. Some research prototypes are not accessible at all (and have been discussed based on papers and screenshots). Other research prototypes are available, and some seem to have users. Some are widely (or at least somewhat) used - showing (perhaps) what's needed to build a Social Web infrastructure for argumentation.

Argumentation support has not yet moved firmly from the academic lab, into the mainstream. While discussion is widespread, argumentation needs are often specific to the reasoning schemes used - which vary by discipline and area. Such constraints simplify the reasoning process for humans as well as for argumentation support. Further, in dialogue, most argumentation happens informally: we can count on our conversational partners to indicate what is missing and to demand that we explain what is unclear to them. It is difficult to systematically indicate assumptions and to make reasoning explicit; while this is needed for ideal reasoning support, it is not feasible or reasonable to expect in everyday discourse. This leads into a discussion of usability.

Usability needs depend on the task at hand and the target audience. Tools for in-depth analysis by experts can be more complex and involved than those for casual use by the general public. E-government and deliberation tools have the strictest usability needs for this reason.

\subsection{Bridging the Social Web and the Semantic Web to manifest the World Wide Argument Web}

To conclude, we discuss the obstacles to manifesting the Social Semantic Argumentation Web, along with a research agenda.

\subsubsection{Problems}

Despite candidate technical architectures for a World Wide Argument Web, the WWAW is not yet viable on the Social Web at large. We notice several interrelated obstacles: first, the existing ontologies are not meant for integrating wide-scale informal discussion; second, current approaches to supporting argumentation generally require substantial human effort; and third, determining the appropriate uses and re-uses for social media posts depends on their context (e.g. the type of discourse).

First, the very informality of social media can make discussions more difficult to integrate. Argumentation is used in many contexts and while formal argumentation can be represented with ontologies such as AIF, 
argumentation on the Social Web can be quite informal, with missing premises and unexpressed argument schemes. While human analysis can sometimes bridge the gap between AIF and the Social Web, (facilitated for instance by tools such as OVA, Section A.29, page 209), more scalable solutions are needed. Several approaches will be needed to more routinely express the existing argumentation on the Social Semantic Web.

Second, most current approaches to supporting argumentation still require substantial human effort; little automatic processing is available. Issues and stances can be categorized by hand, mapped and analyzed, or voted up and down. Tools for certain tasks situational analysis, argumentation reconstruction, and argument mapping - are highly developed. These tools have become, while not mainstream, widely accepted for certain communities. The value of spatial hypertext visualization systems cannot be discounted, and some automation does exist: leveraging human-devised matrices using Bayesian networks (in more advanced versions of $\mathrm{ACH}$ tools like Competing Hypotheses), or summarizing human-answered surveys with argumentation frameworks (Parmenides). Yet since these valueadded tools still require substantial human effort to enter information in particular formats, their very use is an encouraging sign that some forms of argumentation support would be beneficial.

Third, context is important for integrating conversations and claims. One strength of the Semantic Web is in bringing conversations together; this has been very powerful for the Social Semantic Web in general. Yet the rhetorical effect of an argument depends on certain contextual information, such as the surrounding conversation, its participants, and the medium. Extracting and summarizing conversations without this context has risks (i.e. potentially presenting misleading or overstated arguments).

\subsubsection{Research agenda}

To overcome these obstacles and manifest the Social Semantic Argumentation Web, we see a need for various approaches. First, we need ontologies suitable for representing informal Social Web arguments, and to map to these ontologies. Second, to address and reduce the human effort required we also need to motivate participation, and find ways to infer argumentative relationships. Third, we need to further investigate context.

First, we need ontologies that map between the social world and the argumentative world. A modu- lar approach will be needed, reusing existing work, both in domain knowledge and in Social Web modeling, for instance by importing existing ontologies (particularly SIOC). Maximal benefit from a Social Web ontology for argumentation will come from aligning and crosswalking to popular ontologies (especially AIF). Many tools can already output AIF, and analyst-oriented tools can be brought onto the Argument Web with comparatively little effort. Motivated users and defined argumentation schemes ease this process.

Mapping to these ontologies will also leverage the existing human effort already used in argumentation tools. SEAS, for example, already uses argument templating. Such templates appear to be specialized argument schemes, which could be expressed in shared repositories and even classified (for instance using OWL as Avicenna does). Once the argument schemes can be referenced, SEAS might provide another source of AIF data, as well as point to further enrichment needed. The ACH process underlying Competing Hypotheses seems to use a narrower set of reasoning; its data, similarly, might be encompassed by understanding and expressing the $\mathrm{ACH}$ argument scheme. The analyst community is also a good place to start with interface interventions such as using Controlled Natural Language (CNL); whereas on the general Social Web, CNL would restrict input, in analysis tools, CNL might open the vocabulary.

Second, to further address the human effort required, we need to motivate wide-scale participation and improve automatic argument detection. To encourage ongoing human effort, it would be helpful to create a virtuous cycle - by which people benefit from the Argument Web, thereby motivating increased participation. Understanding the niches filled by existing tools, and whether these needs could be fulfilled better by a larger Argument Web, would help in this regard. For instance, while abstract argument schemes may not be well understood by users, Parmenides shows that stepwise processes based on these schemes can be powerful. Opening up the analysis tools, so that a group could view aggregate responses, would take Parmenides to a new level of collaboration. While Parmenides focuses on gathering multiple responses on the same set of issues, a different approach would be to crowdsource work based on an argument scheme. Many groups already do this informally with checklists and procedures, for instance in Wikipedia's article promotion process. Providing templates where users could indicate which critical questions they have asked 
and answered, and at what point in time, might help to distribute and share this work, while making the underlying process more transparent.

Automatic detection of arguments might help further in bootstrapping the existing Social Web into the Argument Web. In the scholarly communication and legal fields, argument detection relies on rhetorical features. Argumentative markers would also help in modifying these argument detection approaches for use on the Social Web. Analyzing existing Social Web corpuses, such as DisputeFinder's claims database and the Discussion Fora from the Aracaria corpus may help in determining such markers. Various corpus-processing techniques and approaches may be useful for detecting argumentation, which shares rhetorical features with other sorts of speech. Linguistic pragmatics dominate in much argumentation, so one form of progress would be to find unassailable features which mark argumentative contexts on the Social Web. Relevant approaches may come from opinion mining [134], question answering and explanation [129], contradiction detection [159], controversy detection [81], persuasion detection $[4,211]$, stance detection [188] and automatically typing links [47].

Third, we need to investigate how to preserve the rhetorical effect of an argument even when it is divorced from its original context. Some aspects of context are straightforward: for instance, items can be confusing or non-sensical when stripped of context. The canonical example from metadata scholars is the "on a horse" [204] problem: in the context of a Theodore Roosevelt collection, the description "on a horse" adequately describes a photo; yet outside of that context it is unclear and diffuse. Other contextual factors include the type of argumentative discourse. The various types of argumentative discourse, shown in Fig. 4 on page 164, vary in the amount of interest and value they generate, outside the immediate context of the discussion. Eristic dialogue, of personal conflicts, for instance, is generally not worth reusing (though it could be used to establish, for instance, who started an argument, or that a dyad should avoid further discussions). Discovery dialogue, on the other hand, which seeks to find and defend a hypothesis, can be useful both for understanding the process undertaken and the outcome.

Support may also depend in part by who reuses discussions - the participants, outside parties, or both and how much support they need at various points in time. Reviews, for instance, are mainly written for an external audience. Blog and microblog posts may be read by others but also searched by the author as a form of externalized memory. There may also be a temporal component: standards bodies use their own discussions in order to make decisions, but after the fact, these discussions may be of considerable interest to non-participants trying to understand why a particular decision was taken. The decision-making associated with discussions may be a particular point where support is needed. Decisions can be taken by groups (e.g. standards bodies) or by individuals (e.g. from reviews) and may depend on a centralized discussion or widely distributed pieces of information.

Overall, there is significant potential for supporting argumentation on the Social Semantic Web, but a large amount of work remains to be done, in creating ontologies, easing human annotation of arguments, improving techniques for detecting and mining argumentation, and in marking the context, pragmatics, and provenance of dialogues. Given the vast amount of commentary on the Social Web - much of it argumentative, persuasive, or opinionated - there is a great need for further research and technical developments to search and organize these discussions with Semantic Web technologies.

\section{Acknowledgements}

This work was supported by Science Foundation Ireland under Grant No. SFI/09/CE/I1380 (Líon2). We are grateful for the extensive comments of the reviewers, Iyad Rahwan, Simon Buckingham Shum, and Fouad Zablith. Thanks also to Ágnes Sándor for her comments which have prompted further clarifications and furthered our thinking. The re-revision of this paper also benefited significantly from conversations with and feedback from Katie Atkinson, Trevor Bench-Capon, and Adam Wyner, thanks to a visit to the University of Liverpool sponsored by the COST Action IC0801 on Agreement Technologies, STSM 1868.

\section{References}

[1] M. Aakhus, Pragmatic Web as communication design practice, in: PragWeb 2006: Proc. of the First International Conference on the Pragmatic Web, 21-23 September 2006, Stuttgart, Germany, Ges. für Informatik, Bonn, 2006.

[2] M. Aakhus and M.G. Benovitz, Argument reconstruction and socio-technical facilitation of large scale argumentation, in: Proc. of the 3rd International Conference on the Pragmatic Web: Innovating the Interactive Society, 2008, pp. 77-81. 
[3] V. Aleven and K.D. Ashley, Evaluating a learning environment for case-based argumentation skills, in: Proc. of the 6th International Conference on Artificial Intelligence and Law, ICAIL 1997, New York, NY, USA, 1997, pp. 170-179.

[4] P. Anand, J. King, J. Boyd-Graber, E. Wagner, C. Martell, D. Oard, and P. Resnik, Believe me - we can do this! Annotating persuasive acts in blog text, in: AAAI Workshop on Computational Models of Natural Argument at the Twenty-Fifth AAAI Conference on Artificial Intelligence, 2011.

[5] M.A. Angrosh, S. Cranefield, and N. Stanger, Ontologybased modelling of related work sections in research articles: Using CRFs for developing semantic data based information retrieval systems, in: iSemantics 2010, 2010.

[6] A. Ankolekar, M. Krötzsch, D.T. Tran, and D. Vrandecic, The two cultures: Mashing up Web 2.0 and the Semantic Web. Journal of Web Semantics 6(1) (2008), 70-75.

[7] K.D. Ashley, Modeling Legal Arguments: Reasoning with Cases and Hypotheticals, MIT Press, Cambridge, MA, USA, 1991.

[8] K.D. Ashley and E.L. Rissland, But, see, accord: Generating blue book citations in HYPO, in: Proc. of the 1st International Conference on Artificial Intelligence and Law, 1987, pp. 6774.

[9] K. Atkinson, T.J.M. Bench-Capon, and D. Walton, Distinctive features of persuasion and deliberation dialogues, Argument \& Computation, 2012, in press.

[10] T. Attwood, A. de Waard, T. Clark, P. Ciccarese, and S. Pettifer, A round-trip to the annotation store: Open, transferable semantic annotation of biomedical publications, in: Beyond the PDF Workshop, University of California San Diego, 2011.

[11] T.J.M. Bench-Capon, Specification and implementation of Toulmin dialogue game, in: Proc. of JURIX, Vol. 98, 1998, pp. 5-20.

[12] T.J.M. Bench-Capon, Persuasion in practical argument using value-based argumentation frameworks, Journal of Logic and Computation 13(3) (2003), 429-448.

[13] T.J.M. Bench-Capon and E.L. Rissland, Back to the future: Dimensions revisited, in: Proc. of JURIX 2001, 2001.

[14] N. Benn, S. Buckingham Shum, J. Domingue, and C. Mancini, Ontological foundations for scholarly debate mapping technology, in: Second International Conference on Computational Models of Argument (COMMA '08), May 2008.

[15] T. Berners-Lee, Linked Data, Design Issues for the World Wide Web, World Wide Web Consortium, 2006. http://www.w3.org/DesignIssues/LinkedData.html

[16] T. Berners-Lee and M. Fischetti, Weaving the Web: The Original Design and Ultimate Destiny of the World Wide Web by its Inventor, Harper Collins Publishers, New York, 1999

[17] T. Berners-Lee, J.A. Hendler, and O. Lassila, The Semantic Web, Scientific American 284(5) (2001), 34-43.

[18] D.A. Black, Introducing HyperNews, Linux Journal (27) (July 1996).

[19] E. Black and A. Hunter, Using enthymemes in an inquiry dialogue system, in: Proc. of the 7th International Joint Conference on Autonomous Agents and Multiagent Systems, Vol. 1, International Foundation for Autonomous Agents and Multiagent Systems, 2008, pp. 437-444.

[20] J. Blythe and Y. Gil, Incremental formalization of document annotations through ontology-based paraphrasing, in: Proc. of the 13th International Conference on World Wide Web, 2004, pp. 455-461.
[21] S. Bocconi, F. Nack, and L. Hardman, Supporting the generation of argument structure within video sequences, in: Proc. of the 16th ACM Conference on Hypertext and Hypermedia, 2005, pp. 75-84.

[22] D. Braybrooke, Introduction to special issue of argumentation originating in a conference at the University of Texas at Austin, Argumentation 17(4) (2003), 361-363.

[23] J.G. Breslin, A. Harth, U. Bojārs, and S. Decker, Towards semantically-interlinked online communities, in: The Semantic Web: Research and Applications, Proceedings of the Second European Semantic Web Conference (ESWC '05), LNCS, Vol. 3532, Springer, 2005, pp. 500-514.

[24] J.G. Breslin, A. Passant, and S. Decker, The Social Semantic Web, Springer, 2009.

[25] S.J. Buckingham Shum, The roots of computer supported argument visualization, in: Visualizing Argumentation: Software Tools for Collaborative and Educational Sense-Making, Springer, 2003, pp. 3-21.

[26] S. Buckingham Shum, Cohere: Towards web 2.0 argumentation, in: Computational Models of Argument: Proceedings of COMMA 2008, Frontiers in Artificial Intelligence and Applications, Vol. 172, IOS Press, 2008.

[27] S. Buckingham Shum, AIF use case: Iraq debate, AIF 2.0 meeting contribution, 2010. http://projects.kmi.open.ac.uk/ hyperdiscourse/docs/AIF-UseCase-v2.pdf.

[28] S. Buckingham Shum and T. Sumner, JIME: An interactive journal for interactive media, First Monday 6(2) (February 2001).

[29] S.J. Buckingham Shum, V. Uren, G. Li, B. Sereno, and C. Mancini, Modeling naturalistic argumentation in research literatures: Representation and interaction design issues, International Journal of Intelligent Systems 22(1) (2007), 1747.

[30] G. Cabanac, M. Chevalier, C. Chrisment, and C. Julien, Social validation of collective annotations: Definition and experiment, Journal of the American Society for Information Science and Technology 61(2) (2010), 271-287.

[31] D.V. Carbogim, D. Robertson, and J. Lee, Argument-based applications to knowledge engineering, The Knowledge Engineering Review 15(2) (2000), 119-149.

[32] J.J. Carroll, C. Bizer, P. Hayes, and P. Stickler, Named graphs, provenance and trust, in: Proc. of the 14th International Conference on World Wide Web, 2004.

[33] J.J. Carroll, I. Dickinson, C. Dollin, D. Reynolds, A. Seaborne, and K. Wilkinson, Jena, in: Proc. of the 13th International World Wide Web Conference - Alternate Track Papers \& Posters - WWW Alt. '04, 2004, p. 74.

[34] D.R. Cartwright, Digital decision-making: Using computational argumentation to support democratic processes, $\mathrm{PhD}$ thesis, University of Liverpool, 62011.

[35] D. Cartwright and K. Atkinson, Political engagement through tools for argumentation, in: Computational Models of Argument: Proc. of COMMA 2008, IOS Press, 2008, pp. 116127.

[36] D. Cartwright and K. Atkinson, Using computational argumentation to support e-participation, IEEE Intelligent Systems 24(5) (2009), 42-52.

[37] D. Cartwright, K. Atkinson, and T. Bench-Capon, Supporting argument in e-democracy, in: Electronic Government: Third International Electronic Democracy (EDEM 2009), 2009, pp. 151-160. 
[38] A.R. Cavagnetto, Argument to foster scientific literacy, Review of Educational Research 80(3) (2010), 336-371.

[39] S. Cayzer, Semantic blogging and decentralized knowledge management, Communications of the ACM 47(12) (2004), 47-52.

[40] H. Chen, AI, e-government, and politics 2.0, IEEE Intelligent Systems 24(5) (2009), 64-86.

[41] C. Chesñevar, J. McGinnis, S. Modgil, I. Rahwan,C. Reed, G. Simari, M. South, G. Vreeswijk, and S. Willmott, Towards an Argument Interchange Format, The Knowledge Engineering Review 21(4) (2006), 293-316.

[42] T. Chklovski, Y. Gil, V. Ratnakar, and J. Lee, TRELLIS: Supporting decision making via argumentation in the Semantic Web, in: Proc. of the Second International Semantic Web Conference, October 2003.

[43] T. Chklovski, V. Ratnakar, and Y. Gil, User interfaces with Semi-Formal representations: A study of designing argumentation structures, in: Proc. of the 10th International Conference on Intelligent User Interfaces, 2005, pp. 130-136.

[44] P. Ciccarese, Scientific discourse relationships ontology specification, Technical Report, Massachusetts General Hospital, December 2008.

[45] P. Ciccarese, M. Ocana, S. Das, and T. Clark, AO: An open Annotation Ontology for science on the Web, in: BioOntologies 2010, 2010.

[46] P. Ciccarese, E. Wu, G. Wong, M. Ocana, J. Kinoshita, A. Ruttenberg, and T. Clark, The SWAN biomedical discourse ontology, Journal of Biomedical Informatics 41(5) (October 2008), 739-751.

[47] C. Cleary and R. Bareiss, Practical methods for automatically generating typed links, in: Proc. of the Seventh ACM Conference on Hypertext, 1996, pp. 31-41.

[48] J.L. De Coi, N.E. Fuchs, K. Kaljurand, and T. Kuhn, Controlled English for reasoning on the Semantic Web, in: Semantic Techniques for the Web - The REWERSE Perspective, Lecture Notes in Computer Science, Vol. 5500, Springer, 2009, pp. 276-308

[49] J. Conklin, Dialogue Mapping: Building Shared Understanding of Wicked Problems, John Wiley and Sons, Ltd., 2005.

[50] S. Das, M. Rogan, H. Kawadler, S. Brin, S. Corlosquet, and T. Clark, PD Online: A case study in scientific collaboration on the Web, in: Workshop on the Future of the Web for Collaborative Science, 19th International World Wide Web Conference, 2010.

[51] N. Diakopoulos, S. Goldenberg, and I. Essa, Videolyzer: Quality analysis of online informational video for bloggers and journalists, in: Proc. of the 27th International Conference on Human Factors in Computing Systems, 2009, pp. 799808.

[52] P.M. Dung, On the acceptability of arguments and its fundamental role in nonmonotonic reasoning, logic programming and n-person games, Artificial Intelligence 77(2) (1995), 321-357.

[53] P.E. Dunne, S. Doutre, and T.J.M. Bench-Capon, Discovering inconsistency through examination dialogues, in: IJCAI, L.P. Kaelbling and A. Saffiotti, eds, Professional Book Center, 2005, pp. 1680-1681.

[54] S. Edelston Toulmin, The Uses of Argument, University Press, Cambridge [Eng.], 1958.

[55] F.H van Eemeren, ed, Advances in Pragma-Dialectics, SicSat/Vale Press, 2002.
[56] F.H. van Eemeren and R. Grootendorst, Speech Acts in Argumentative Discussions: A Theoretical Model for the Analysis of Discussions Directed Toward Solving Conflicts of Opinion, de Gruyter Mouton, June 1984.

[57] F.H. van Eemeren and R. Grootendorst, Argumentation, Communication and Fallacies: A Pragma-Dialectical Perspective, Erlbaum, 1992.

[58] F.H. van Eemeren and R. Grootendorst, A Systematic Theory of Argumentation: The Pragma-Dialectical Approach, Cambridge University Press, 2004.

[59] F.H. van Eemeren, R. Grootendorst, and F. Snoeck Henkemans, Argumentation: Analysis, Evaluation, Presentation, Erlbaum, 2002.

[60] R. Ennals, D. Byler, J.M. Agosta, and B. Rosario, What is disputed on the Web? in: WWW 2010, 2010.

[61] R. Ennals, B. Trushkowsky, and J.M. Agosta, Highlighting disputed claims on the Web, in: WICOW 2010 at WWW 2010 , 2010 .

[62] ESTRELLA Project, Deliverable 1.4: OWL ontology of basic legal concepts (LKIF-Core), Deliverable IST-2004-027655, University of Amsterdam, January 2007.

[63] S. Faridani, E. Bitton, K. Ryokai, and K. Goldberg, Opinion Space: A scalable tool for browsing online comments, in: Proc. of the 28th International Conference on Human Factors in Computing Systems, CHI '10, 2010, pp. 1175-1184.

[64] G. Fischer, A.C. Lemke, R. McCall, and A.I. Morch, Making argumentation serve design, Human-Computer Interaction 6(3) (1991), 393-419.

[65] J.S. Fishkin, Democracy and Deliberation: New Directions for Democratic Reform, Yale University Press, 1991.

[66] N.E. Fuchs, K. Kaljurand, and G. Schneider, Attempto Controlled English meets the challenges of knowledge representation, reasoning, interoperability and user interfaces, in: FLAIRS Conference, 2006, pp. 664-669.

[67] A. Garcia Castro, A. Norena, A. Betancourt, and M.A. Ragan, Cognitive support for an argumentative structure during the ontology development process, in: Proc. of the 9th International Protégé Conference, Stanford, California, July 2006.

[68] M.A. Gerosa, M.G. Pimentel, H. Fuks, and C. Lucena, Analyzing discourse structure to coordinate educational forums, in: Intelligent Tutoring Systems 2004, LNCS, Vol. 3220, Springer-Verlag, 2004, pp. 23-36.

[69] Y. Gil and V. Ratnakar, TRELLIS: An interactive tool for capturing information analysis and decision making, Internal Project Report, USC Information Sciences Institute, 2001. http://www.isi.edu/expect/web/trellis/Trellis-8-01.pdf.

[70] Y. Gil and V. Ratnakar, Trusting information sources one citizen at a time, in: Proc. of the First International Conference on The Semantic Web, Springer-Verlag, 2002, pp. 162-176.

[71] T.F. Gordon, H. Prakken, and D. Walton, The Carneades model of argument and burden of proof, Artificial Intelligence 171(10-15) (July 2007), 875-896.

[72] W.S. Grennan, Informal Logic: Issues and Techniques, McGill Queens University Press, Montreal, Quebec, 1997.

[73] H.P. Grice, Logic and conversation, in: Syntax and Semantics, Vol. 3, 1975, pp. 41-58.

[74] L. Groarke, Informal logic, in: The Stanford Encyclopedia of Philosophy, E.N. Zalta, ed, Spring 2012 edn, 2012.

[75] C. Grover, B. Hachey, and C. Korycinski, Summarising legal texts: Sentential tense and argumentative roles, in: Proc. of the Text Summarization Workshop at HLT-NAACL 2003, Hu- 
man Language Technology Conference of the North American Chapter of the Association for Computational Linguistics, Vol. 5, 2003, pp. 33-40.

[76] T. Groza, S. Handschuh, J.G. Breslin, and S. Decker, An abstract framework for modeling argumentation in virtual communities, International Journal of Virtual Communities and Social Networking 1 (2009), 35-47.

[77] T. Groza, S. Handschuh, K. Möller, and S. Decker, SALT - Semantically Annotated LaTeX for scientific publications, in: The Semantic Web: Research and Applications, Proc. of ESWC 2007, LNCS, Springer, 2007, pp. 518-532.

[78] T.R. Gruber, Towards principles for the design of ontologies used for knowledge sharing, International Journal HumanComputer Studies 43(5-6) (1995), 907-928.

[79] T.R. Gruber, Collective knowledge systems: Where the Social Web meets the Semantic Web, Journal of Web Semantics 6(1) (2008), 4-13.

[80] A. Gürkan, L. Iandoli, M. Klein, and G. Zollo, Mediating debate through on-line large-scale argumentation: Evidence from the field, Information Sciences 180(19) (2010), 36863702 .

[81] K. Gyllstrom and M.-F. Moens, Clash of the typings: Finding controversies and children's topics within queries, in: Proc. of the 33rd European Conference on Advances in Information Retrieval, ECIR'11, Springer-Verlag, 2011, pp. 80-91.

[82] S. Heras, Case-based argumentation in agent societies, $\mathrm{PhD}$ thesis, Universitat Politècnica de València, 2011.

[83] S. Heras, K. Atkinson, V. Botti, F. Grasso, V. Juliana, and P. McBurney, Applying argumentation to enhance dialogues in social networks, in: Proc. of the IJCAI Workshop on Computational Models of Natural Argument (CMNA), 2010.

[84] S. Heras, K. Atkinson, V. Botti, F. Grasso, V. Juliana, and P. McBurney, How argumentation can enhance dialogues in social networks, in: Computational Models of Argument Proc. of COMMA 2010, Frontiers in Artificial Intelligence and Applications, Vol. 216, IOS Press, 2010, pp. 267-274.

[85] S. Heras, V. Botti, and V. Juliana, On a Computational Argumentation Framework for Agent Societies, LNCS, Vol. 6614, Springer-Verlag, New York, 2011, pp. 123-140.

[86] M.H.G. Hoffmann, Logical argument mapping: A cognitivechange-based method for building common ground, in: Proc. of the 2nd International Conference on Pragmatic Web, ICPW '07, 2007, pp. 41-47.

[87] M. Hoffmann, Logical argument mapping (LAM) - A manual, Working Paper, Georgia Tech, 2011. http://www.spp.gatech.edu/faculty/workingpapers/wp50.pdf.

[88] K. Hyland, Metadiscourse, 1st edn, Continuum, 2005.

[89] J.E. Introne, Adaptive mediation in groupware, $\mathrm{PhD}$ thesis, Brandeis University, 2008.

[90] J.E. Introne, Supporting group decisions by mediating deliberation to improve information pooling, in: Proc. of the ACM 2009 International Conference on Supporting Group WorkGROUP '09, 2009, p. 189.

[91] A.P.J. Jarczyk, P. Löffler, and F.M. Shipman III, Design rationale for software engineering: A survey, in: Proc. of the Twenty-Fifth Hawaii International Conference on System Sciences, 1992, Vol. ii, 1992, pp. 577-586.

[92] M. Jeong, C.-Y. Lin, and G.G. Lee, Semi-supervised speech act recognition in emails and forums, in: EMNLP: Proc. of the Conference on Empirical Methods in Natural Language Processing, 2009, pp. 1250-1259.
[93] D.R. Karger and D. Quan, What would it mean to blog on the Semantic Web? in: The Semantic Web - ISWC 2004, Springer, 2004, pp. 214-228.

[94] J. Katzav and C. Reed, On argumentation schemes and the natural classification of arguments, Argumentation 18(2) (June 2004), 239-259.

[95] J. Katzav, C. Reed, and G. Rowe, Argument research corpus, in: Proc. of the 2003 Conference on Practical Applications in Language and Computers, Peter Lang, 2003, pp. 229-239.

[96] P.A. Kirschner, S.J. Buckingham Shum, and C.S. Carr, Visualizing Argumentation: Software Tools for Collaborative and Educational Sense-Making, 1st edn, Springer, 2003.

[97] M. Klein, Achieving collective intelligence via large-scale on-line argumentation, Technical Report, Research Paper No. 4647-07, CCI Working Paper No. 2007-001, MIT Sloan School of Management.

[98] M. Klein, The MIT collaboratorium: Enabling effective largescale deliberation for complex problems, SSRN eLibrary, (Research Paper No. 4679-08), December 2007.

[99] G. Klyne and J.J. Carroll, Resource Description Framework (RDF): Concepts and abstract syntax, W3C Recommendation 10 February 2004, World Wide Web Consortium, 2004. http://www.w3.org/TR/rdf-concepts/.

[100] A. Knott and T. Sanders, The classification of coherence relations and their linguistic markers: An exploration of two languages, Journal of Pragmatics 30 (1998), 135-175.

[101] K. Korta and J. Perry, Pragmatics, in: The Stanford Encyclopedia of Philosophy, E.N. Zalta, ed, Summer 2011 edn, 2011.

[102] T. Kriplean, J.T. Morgan, D. Freelon, A. Borning, and L. Bennett, ConsiderIt: Improving structured public deliberation, in: Proc. of the 2011 Annual Conference Extended Abstracts on Human Factors in Computing Systems, CHI EA '11, 2011, pp. 1831-1836.

[103] T. Kriplean, J. Morgan, D. Freelon, A. Borning, and L. Bennett, Supporting reflective public thought with ConsiderIt, in: Proc. of the ACM 2012 Conference on Computer Supported Cooperative Work, CSCW'12, New York, NY, USA, 2012, pp. 265-274.

[104] M. Krötzsch, D. Vrandečić, M. Völkel, H. Haller, and R. Studer, Semantic Wikipedia, Journal of Web Semantics 5(4) (2007), 251-261.

[105] W. Kunz and H.W.J. Rittel, Issues as elements of information systems, Working Paper 131, Center for Planning and Development Research, University of California at Berkeley, Berkeley, CA, 1970.

[106] C. Lange, Semantic Web collaboration on semiformal mathematical knowledge, $\mathrm{PhD}$ thesis, Jacobs University, School of Engineering and Science, Bremen, Germany, Germany, 2010. https://svn.kwarc.info/repos/swim/doc/phd/phd.pdf.

[107] C. Lange, U. Bojārs, T. Groza, J.G. Breslin, and S. Handschuh, Expressing argumentative discussions in social media sites, in: Proc. of the ISWC2008 Workshop on Social Data on the Web (SDoW2008), October 2008.

[108] C. Lange, T. Hastrup, and S. Corlosquet, Arguing on issues with mathematical knowledge items in a semantic wiki, in: LWA: Lernen, Wissensentdeckung und Adaptivität, Technical Report, Vol. 448, Department of Computer Science, University of Würzburg, Germany, 2008, pp. 41-48.

[109] I.A. Letia and A. Groza, Contextual extension with concept maps in the Argument Interchange Format, in: Argumentation in Multi-Agent Systems, Vol. 5384, Springer, 2009. 
[110] A. De Liddo and S. Buckingham Shum, Cohere: A prototype for contested collective intelligence, in: Collective Intelligence in Organizations - Toward a Research Agenda, 2010.

[111] C. Lópea, P. Inostroza, L.M. Cysneiros, and H. Astudillo, Visualization and comparison of architecture rationale with Semantic Web technologies, The Journal of Systems and Software 82(8) (2009), 1198-1210.

[112] R.P. Lourenço and J.P. Costa, A note on democracy and group support systems concepts, in: CISTI: 2010 5th Iberian Conference on Information Systems and Technologies, 2010, pp. $1-6$.

[113] J.D. Lowrance, Graphical manipulation of evidence in structured arguments, Law, Probability and Risk 6(1-4) (2007), 225.

[114] J. Lowrance, I. Harrison, A. Rodriguez, E. Yeh, T. Boyce, J. Murdock, J. Thomere, and K. Murray, Template-based structured argumentation, in: Knowledge Cartography, Advanced Information and Knowledge Processing, Springer, 2008, pp. 307-334

[115] A. Macintosh, Moving toward "Intelligent" policy development? IEEE Intelligent Systems 24(5) (2011), 79-82.

[116] A. Macintosh, T.F. Gordon, and A. Renton, Providing argument support for e-participation, Journal of Information Technology \& Politics 6(1) (2009), 43-59.

[117] C. Mancini and S. Buckingham Shum, Cognitive coherence relations and hypertext: From cinematic patterns to scholarly discourse, in: Proc. of the 12th ACM Conference on Hypertext and Hypermedia, Arhus, Denmark, 2001, pp. 165-174.

[118] C. Mancini and S.J. Buckingham Shum, Modelling discourse in contested domains: A semiotic and cognitive framework, International Journal of Human-Computer Studies 64(11) (November 2006), 1154-1171.

[119] W.C. Mann and S.A. Thompson, Rhetorical structure theory: Toward a functional theory of text organization, Text $\mathbf{8}(3)$ (1988), 243-281.

[120] D. Marcu, Building up rhetorical structure trees, in: AAAI-96 Proceedings, 1996.

[121] D. Marcu, The Theory and Practice of Discourse Parsing and Summarization, The MIT Press, November 2000.

[122] P. McBurney and S. Parsons, Dialogue games for agent argumentation, Argumentation in Artificial Intelligence, 2009, pp. 261-280.

[123] H.M. Mentis, P.M. Bach, B. Hoffman, M.B. Rosson, and J.M. Carroll, Development of decision rationale in complex group decision making, in: Proc. of the 27th International Conference on Human Factors in Computing Systems, 2009, pp. 1341-1350.

[124] R. Mochales Palau, Automatic detection and classification of argumentation in a legal case, $\mathrm{PhD}$ thesis, K.U. Leuven, Faculty of Engineering Science, July 2011.

[125] R. Mochales-Palau and M.-F. Moens, Automatic argumentation detection and its role in law and the Semantic Web, in Law, Ontologies and the Semantic Web - Channelling the Legal Information Flood, Frontiers in Artificial Intelligence and Applications, Vol. 188, IOS Press, 2009, pp. 115-129.

[126] R. Mochales and M.-F.F. Moens, Argumentation mining, Artificial Intelligence and Law 19(1) (2011), 1-22.

[127] S. Modgil and J. McGinnis, Towards characterising argumentation based dialogue in the Argument Interchange Format, in: Argumentation in Multi-Agent Systems, Vol. 4946, Springer, 2008.
[128] A. de Moor and M. Aakhus, Argumentation support: From technologies to tools, Communications of the ACM 49(3) (2006), 93-98.

[129] B. Moulin, H. Irandoust, M. Bélanger, and G. Desbordes, Explanation and argumentation capabilities: Towards the creation of more persuasive agents, Artificial Intelligence Review 17 (2002), 169-222.

[130] S.E. Newman and C.C. Marshall, Pushing Toulmin too far: Learning from an argument representation scheme, Technical Report SSL-92-45, Xerox PARC, 1992. http://www.csdl.tamu.edu/ marshall/toulmin.pdf.

[131] A. Okada, S.J. Buckingham Shum, and T. Sherborne, Knowledge Cartography: Software Tools and Mapping Techniques, Springer, 2008.

[132] T. O'Reilly, What is Web 2.0: Design Patterns and Business Models for the Next Generation of Software, 30 September 2005. http://www.oreillynet.com/lpt/a/6228.

[133] OWL 2 Web Ontology Language: Document Overview, W3C Recommendation 27 October 2009, World Wide Web Consortium, 2009. http://www.w3.org/TR/ow12-overview/.

[134] B. Pang and L. Lee, Opinion mining and sentiment analysis, Foundations and Trends in Information Retrieval 2(1-2) (2008), 1-135.

[135] J. Park, Boundary infrastructures for IBIS federation: Design rationale, implementation, and evaluation, $\mathrm{PhD}$ thesis Proposal KMI-10-01, The Open University, Milton Keyes, 2010.

[136] P. Pasquier, I. Rahwan, F. Dignum, and L. Sonenberg, Argumentation and persuasion in the cognitive coherence theory, in: Proc. of the 2006 Conference on Computational Models of Argument: Proc. of COMMA 2006, Amsterdam, The Netherlands, IOS Press, 2006, pp. 223-234.

[137] A. Passant, Semantic Web Technologies for Enterprise 2.0, IOS Press, 2010.

[138] A. Passant, P. Ciccarese, J.G. Breslin, and T. Clark, SWAN/SIOC: Aligning scientific discourse representation and social semantics, in: Proc. of the Workshop on Semantic Web Applications in Scientific Discourse (SWASD 2009), at (ISWC-2009), Vol. 523, 2009. CEUR-WS.org.

[139] C. Perelman and L. Olbrechts-Tyteca, The New Rhetoric: A Treatise on Argumentation, University of Notre Dame Press, June 1969.

[140] S. Peroni and D. Shotton, FaBiO and CiTO: Ontologies for describing bibliographic resources and citations, Web Semantics: Science, Services and Agents on the World Wide Web (2012), doi:10.1016/j.websem.2012.08.001.

[141] H.S. Pinto, C. Tempich, and S. Staab, DILIGENT: Towards a fine-grained methodology for DIstributed, loosely-controlled and evolvInG engingeering of oNTologies, in: Proc. of the 16th European Conference on Artificial Intelligence (ECAI 2004), August 22nd-27th, August 2004, pp. 393-397.

[142] H. Prakken, Formal systems for persuasion dialogue, The Knowledge Engineering Review 21(2) (2006), 163-188.

[143] I. Rahwan, Mass argumentation and the Semantic Web, Web Semantics: Science, Services and Agents on the World Wide Web 6(1) (February 2008), 29-37.

[144] I. Rahwan and B. Banihashemi, Arguments in OWL: A progress report, in: Computational Models of Argument: Proceedings of COMMA 2008, IOS Press, 2008, pp. 297310.

[145] I. Rahwan, B. Banihashemi, C. Reed, D. Walton, and S. Abdallah, Representing and classifying arguments on the Se- 
mantic Web, The Knowledge Engineering Review 26(04) (12 2011), 487-511.

[146] I. Rahwan and G. Simari, Argumentation in Artificial Intelligence, Springer, 2009.

[147] I. Rahwan, F. Zablith, and C. Reed, Laying the foundations for a World Wide Argument Web, Artificial Intelligence 171(10-15) (July 2007), 897-921.

[148] I. Rahwan, F. Zablith, and C. Reed, Towards large scale argumentation support on the Semantic Web, in: Proc. of the 22nd National Conference on Artificial Intelligence, Vol. 2, AAAI Press, 2007, pp. 1446-1451.

[149] A. Ravenscroft, S. Wells, M. Sagar, and C. Reed, Mapping persuasive dialogue games onto argumentation structures, in: Symposium on Persuasive Technology \& Digital Behaviour Intervention at AISB: Artificial Intelligence and Simulation of Behaviour, April 2009.

[150] RDF Vocabulary Description Language 1.0: RDF Schema, W3C Recommendation 10 February 2004, World Wide Web Consortium, 2004. http://www.w3.org/TR/rdf-schema/.

[151] C. Reed, Representing dialogic argumentation, KnowledgeBased Systems 19(1) (March 2006), 22-31.

[152] C. Reed and G. Rowe, Araucaria: Software for argument analysis, diagramming and representation, International Journal on Artificial Intelligence Tools 13(4) (2004), 961-979.

[153] C. Reed and C.W. Tindale, Dialectics, dialogue and argumentation: An examination of Douglas Walton's theories of reasoning and argument, College Publications, London, 2010.

[154] C. Reed, D. Walton, and F. Macagno, Argument diagramming in logic, law and Artificial Intelligence, The Knowledge Engineering Review 22(01) (2007), 87-109.

[155] C. Reed and S. Wells, Dialogical argument as an interface to complex debates, IEEE Intelligent Systems 22(6) (2007), 60-65.

[156] C. Reed, S. Wells, K. Budzynska, and J. Devereux, Building arguments with argumentation: The role of illocutionary force in computation models of argument, in: Computational Models of Argument - Proc. of COMMA 2010, Frontiers in Artificial Intelligence and Applications, Vol. 216, IOS Press, 2010, pp. 415-426.

[157] C. Reed, S. Wells, J. Devereux, and G. Rowe, AIF+: Dialogue in the argument interchange format, in: Computational Models of Argument: Proc. of COMMA 2008, IOS Press, 2008, pp. 311-323.

[158] A. Ritter, C. Cherry, and B. Dolan, Unsupervised modeling of Twitter conversations, in: Proc. of Human Language Technologies: The 11th Annual Conference of the North American Chapter of the Association for Computational Linguistics, 2010.

[159] A. Ritter, D. Downey, S. Soderland, and O. Etzioni, It's a contradiction - no, it's not: A case study using functional relations, in: EMNLP: Proc. of the Conference on Empirical Methods in Natural Language Processing, Association for Computational Linguistics, 2008, pp. 11-20.

[160] G.W.A. Rowe, C.A. Reed, and J. Katzav, Araucaria: Marking up argument, in: European Conference on Computing and Philosophy, 2003.

[161] M. Samwald and K.-P. Adlassnig, The bio-zen plus ontology, Applied Ontology 3(4) (2008), 213-217.

[162] T.J.M. Sanders, W.P.M. Spooren, and L. Noordman, Towards a taxonomy of coherence relations, Discourse Processes $\mathbf{1 5}$ (1992), 1-35.
[163] Á. Sándor, Using the author's comments for knowledge discovery, in: Semaine de la Connaissance, Atelier Texte et Connaissance, Nantes, France, 2006.

[164] O. Scheuer, F. Loll, N. Pinkwart, and B. McLaren, Computersupported argumentation: A review of the state of the art, International Journal of Computer-Supported Collaborative Learning 5(1) (2010), 43-102.

[165] J. Schneider, A. Passant, and S. Decker, Deletion discussions in Wikipedia: Decision factors and outcomes, in: 8th International Symposium on Wikis and Open Collaboration, WikiSym 2012, 2012

[166] D.C. Schneider, C. Voigt, and G. Betz, ArguNet - a software tool for collaborative argumentation analysis and research, in: Proc. of the IJCAI Workshop on Computational Models of Natural Argument (CMNA), 2007.

[167] D. Schober, J. Malone, and R. Stevens, Practical experiences in concurrent, collaborative ontology building using Collaborative Protégé, in: Proc. of the International Conference on Biomedical Ontology, Buffalo, NY, 2009, Nature Precedings. http://precedings.nature.com/documents/3517/version/2.

[168] W. Schuler and J.B. Smith, Author's argumentation assistant (AAA): A hypertext-based authoring tool for argumentative texts, in: Hypertext: Concepts, systems and applications (Proc. of the ECHT'90 European Conference on Hypertext), Cambridge University Press, 1992, pp. 137-151.

[169] J.R. Searle, Speech Acts, Cambridge University Press, New York, 1969

[170] F.M. Shipman and C.C. Marshall, Formality considered harmful: Experiences, emerging themes, and directions on the use of formal representations in interactive systems, Computer Supported Cooperative Work 8(4) (December 1999), 333-352.

[171] D. Shotton, CiTO, the Citation Typing Ontology, and its use for annotation of reference lists and visualization of citation networks, in: Bio-Ontologies 2009, a Special Interest Group Meeting at ISMB 2009, 2009.

[172] D. Shotton, CiTO, the Citation Typing Ontology, Journal of Biomedical Semantics 1(Suppl 1) (2010), S6.

[173] E. Sirin, B. Parsia, B. Cuenca Grau, A. Kalyanpur, and Y. Katz, Pellet: A practical OWL-DL reasoner, Web Semantics: Science, Services and Agents on the World Wide Web 5(2) (2007), 51-53.

[174] M. Snaith, J. Devereux, J. Lawrence, and C. Reed, Pipelining argumentation technologies, in: Computational Models of Argument - Proc. of COMMA 2010, Frontiers in Artificial Intelligence and Applications, Vol. 216, IOS Press, 2010.

[175] M. Snaith, J. Lawrence, and C. Reed, Mixed initiative argument in public deliberation, in: Proc. of the Fourth International Conference on Online Deliberation, 2010.

[176] M. South, G. Vreeswijk, and J. Fox, Dungine: A JAVA Dung reasoner, in: Proc. of the 2008 Conference on Computational Models of Argument (COMMA 2008), IOS Press, 2008, pp. 360-368.

[177] SPARQL query language for RDF, W3C Recommendation 15 January 2008, World Wide Web Consortium, 2008. http://www.w3.org/TR/rdf-sparql-query.

[178] N.A. Streitz, J. Hannemann, and M. Thüring, From ideas and arguments to hyperdocuments: Travelling through activity spaces, in: Proc. of the Second Annual ACM Conference on Hypertext, HYPERTEXT' '89, 1989, pp. 343-364. 
[179] D.D. Suthers, Empirical studies of the value of conceptually explicit notations in collaborative learning, in: Knowledge Cartography, Advanced Information and Knowledge Processing, Springer, 2008, pp. 1-24.

[180] M. Taboada and W.C. Mann, Applications of Rhetorical Structure Theory, Discourse Studies 8(4) (August 2006), 567-588

[181] C. Tempich, H.S. Pinto, Y. Sure, and S. Staab, An argumentation ontology for DIstributed, loosely-controlled and evolvInG engineering processes of oNTologies (DILIGENT), in: The Semantic Web: Research and Applications, Springer, 2005, pp. 241-256.

[182] S. Teufel and M. Moens, Summarizing scientific articles: Experiments with relevance and rhetorical status, Computational Linguist. 28(4) (2002), 409-445.

[183] P. Thagard, Explanatory coherence, Behavioral and Brain Sciences 12(3) (1989), 435-502.

[184] F. Toni and P. Torroni, Bottom-up argumentation, in: First International Workshop on the Theory and Applications of Formal Argumentation, 2011.

[185] P. Torroni, F. Chesani, and M. Gavanelli, Argumentation in the Semantic Web, IEEE Intelligent Systems 22(6) (2007), 66-74.

[186] D.P. Twitchell, M. Adkins, J.F. Nunamaker Jr., and J.K. Burgoon, Using Speech Act Theory to model conversations for automated classification and retrieval, in: (LAP'04): Proc. of the 9th International Working Conference on the LanguageAction Perspective on Communication Modelling, 2004, pp. 121-129.

[187] M. Tzagarakis, N. Karousos, N. Karacapilidis, and D. Nousia, Unleashing argumentation support systems on the Web: The case of CoPe_it! in: Web Science 2009, Athens, Greece, March 2009.

[188] M.A. Walker, P. Ananda, R. Abbott, J.E. Fox Tree, C. Martell, and J. King, That's your evidence?: Classifying stance in online political debate, Decision Support Systems, 2012, in press, doi:10.1016/j.dss.2012.05.032.

[189] D. Walton, Plausible Argument in Everyday Conversation, SUNY Press, 1992

[190] D. Walton, Argumentation Schemes for Presumptive Reasoning, Routledge, 1995.

[191] D.N. Walton, Commitment in Dialogue: Basic Concepts of Interpersonal Reasoning, State University of New York Press, Albany, 1995.

[192] D. Walton, How can logic best be applied to arguments? Logic Journal of the IGPL 5(4) (July 1997), 603614.

[193] D. Walton, Fundamentals of Critical Argumentation, Cambridge University Press, October 2005.

[194] D. Walton, Dialog Theory for Critical Argumentation, John Benjamins, 2007

[195] D. Walton, Informal Logic: A Pragmatic Approach, Cambridge University Press, 2nd edn, 2008.

[196] D. Walton, Types of dialogue and burdens of proof, in: Computational Models of Argument - Proc. of COMMA 2010, Frontiers in Artificial Intelligence and Applications, Vol. 216 IOS Press, 2010, pp. 13-24.

[197] D. Walton, K. Atkinson, T. Bench-Capon, A. Wyner, and D.Cartwright, Argumentation in the framework of deliberation dialogue, Arguing Global Governance: Agency, Lifeworld and Shared Reasoning, 2010, p. 210.
[198] D. Walton, C. Reed, and F. Macagno, Argumentation Schemes, 1st edn, Cambridge University Press, August 2008

[199] B. Warnick, Rhetoric Online: Persuasion and Politics on the World Wide Web, Peter Lang, 2007.

[200] B. Webber, M. Egg, and V. Kordoni, Discourse structure and language technology, Natural Language Engineering, FirstView Article, 2011, pp. 1-54.

[201] S. Wells, C. Gourlay, and C. Reed, Argument blogging, in: Proc. of the IJCAI Workshop on Computational Models of Natural Argument (CMNA), 2009.

[202] S. Wells and C. Reed, MAgtALO: An agent-based system for persuasive online interaction, in: AISB 2008: Artificial Intelligence and Simulation of Behaviour, 2008.

[203] S. Wells and C. Reed, A domain specific language for describing diverse systems of dialogue, Journal of Applied Logic, 2011, to appear.

[204] R. Wendler, The eye of the beholder: Challenges of image description and access at Harvard, in: Metadata in Practice, ALA Editions, 2004, pp. 51-69.

[205] T. Winograd, A Language/Action perspective on the design of cooperative work, Human-Computer Interaction 3(1) (March 1987), 3-30.

[206] A. Wyner and T. van Engers, A framework for enriched, controlled on-line discussion forums for e-government, in: $e G O V$ 2010, 2010, pp. 1-11.

[207] A. Wyner, T. Van Engers, and K. Bahreini, From policymaking statements to first-order logic, in: Electronic Government and the Information System Perspective, LNCS, Vol. 6267, Springer, 2010, pp. 47-61.

[208] A. Wyner, R. Mochales-Palau, M.-F. Moens, and D. Milward, Approaches to text mining arguments from legal cases, in: $\mathrm{Se}$ mantic Processing of Legal Texts, Lecture Notes in Computer Science, Vol. 6036, Springer, 2010, pp. 60-79.

[209] A. Wyner, J. Schneider, K. Atkinson, and T. Bench-Capon, Semi-automated argumentative analysis of online product reviews, in: Proc. of the 2012 Conference on Computational Models of Argument (COMMA 2012).

[210] K.-P. Yee, Zest: Discussion mapping for mailing lists, in: Proc. of the 2002 ACM Conference on Computer-Supported Cooperative Work, 2002.

[211] J. Young, C. Martell, P. Anand, P. Ortiz, and H.T. Gilbert IV, A microtext corpus for persuasion detection in dialog, in: Workshops at the Twenty-Fifth AAAI Conference on Artificial Intelligence, 2011.

[212] F. Zablith, ArgDF: Arguments on the Semantic Web, Master's, The British University in Dubai Jointly with The University of Edinburgh, February 2007. http://www.argdf.org/source/.

\section{Appendix}

\section{A. Tools}

\section{A.1. List of social and Semantic Web tools considered}

In this section we draw from our review of thirtyseven online argumentation tools: AGORA: Participate - Deliberate, ArgDF, Arguehow, Argument Blog- 


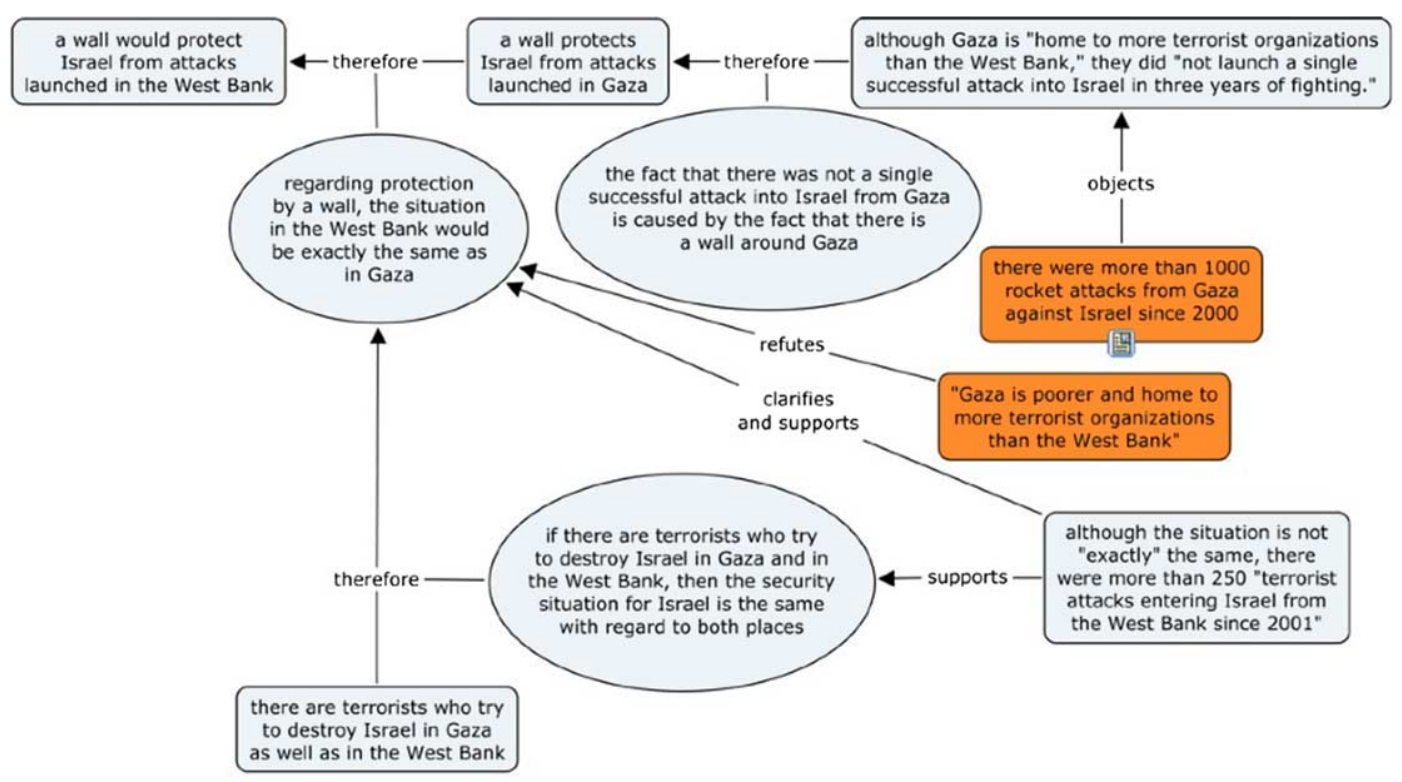

Fig. 24. A sample LAM map, from [86]. (Color figure online)

ging, Argumentum, Argumentations.com, Argunet, Avicenna, bCisiveOnline, Belvedere, Cabanac's annotation system, Climate CoLab, Cohere, Competing Hypotheses, ConsiderIt, ConvinceMe, CoPe_it!, CreateDebate, Debate.org, Debategraph, Debatepedia, Debatewise, DiscourseDB, Dispute Finder, Hypernews, Living Vote, MAgTALO, Opinion Space, Online Visualisation of Arguments, Parmenides, PDOnline, REASON, Riled Up!, SEAS, Trellis, TruthMapping, and Videolyzer. For further details about our inclusion criteria, see Section 9, page 179.

\section{A.2. AGORA: Participate-Deliberate}

Michael Hoffman's system, AGORA: Participate Deliberate [86], uses Logical Argument Mapping [87] (Fig. 24), providing support for representing deductively valid arguments, using one of seven schemes: modus ponens; modus tollens; disjunctive syllogism; not-both syllogism; conditional syllogism; equivalence; and constructive dilemma. It relies on concept mapping software called CmapTools ${ }^{75}$.

\section{A.3. $\operatorname{Arg} D F$}

$\operatorname{ArgDF}^{76}$ is a Semantic Web-based argumentation system using the AIF-RDF ontology described above

\footnotetext{
${ }^{75}$ http://cmap.ihmc.us/

${ }^{76} \mathrm{http}: / / \operatorname{argdf} . \mathrm{org} /$
}

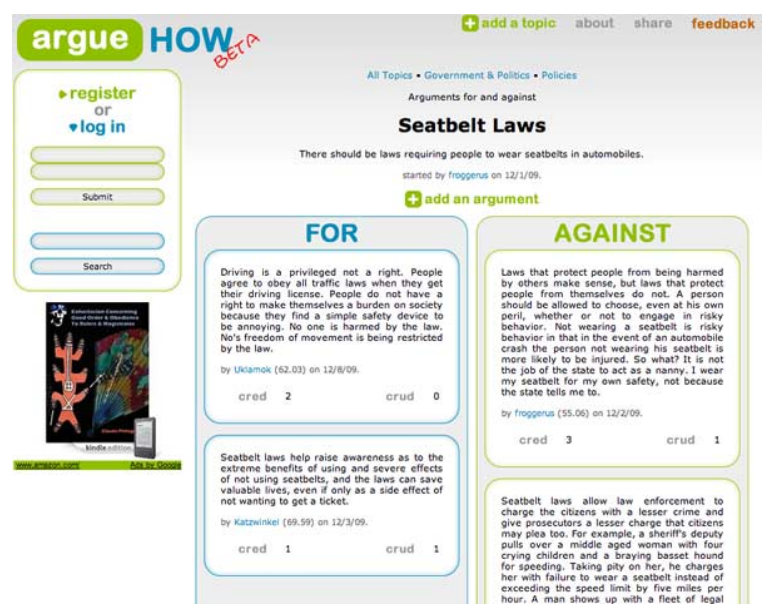

Fig. 25. ArgueHow offers structured discussion. (Color figure online)

[147,148,212]. ArgDF uses Sesame RDF for storage and querying and Phesame for communicating with the Sesame through PHP pages.

\section{A.4. ArgueHow}

ArgueHow $^{77}$ (Fig. 25) is a argument-based discussion board aimed at a general audience. Its purpose is to help find the best points supporting a position. Discussion points are sorted by votes for ('Creds') and against ('Cruds') them. ArgueHow has a unique way

${ }^{77}$ http://arguehow.com/ 
of handling reputation: users start with a reputation of 50, which increases or decreases according to the votes their points accrue. Votes are weighted: for instance, points with 10 'cred' or 'crud' votes change less in response to further votes, and votes on users' first 20 discussion points affect their reputation less than later contributions, allowing them to learn the system.

\section{A.5. Argument blogging}

The idea of argument blogging was proposed by Wells, Gourlay and Reed [201] as a way to bring blogs into the WWAW, based on standard Web technologies, and augmented by argument specific technologies. In addition to AIF, argument blogging relies on the AIF Database (AIFDB) and Dialog Game Description Language (DGDL). AIFDB is a MySQL database for storing AIF documents which can be serialized as RDF and accessed via a RESTful Web service. DGDL $[149,203]$ is a grammar for describing the rules of dialogue games.

Argument blogging uses text from the current Web as a departure point for the WWAW. When browsing the Web, users select text and click a JavaScript bookmarklet, to indicate whether they will attack an inference, support or refute the selected text. This generates a fragment of embeddable JavaScript the user can paste onto his/her blog. Once a blogger opts in to the WWAW by adding JavaScript to a webpage, the page displays a badge which links back to argument blogging server, where the distributed dialog can be visualized or exported as text.

Earlier work on semantic blogging predates the WWAW but focused more attention on the visualization of reply graphs of messages from multiple blogs [93] or the possibilities for inference [39].

\section{A.6. Argumentum}

Argumentum $^{78}$ is an argument-based discussion site aimed at airing discussions. Debaters add topics and their arguments are colored to indicate the supporting (green) and opposing (red) arguments (Fig. 26). Comments, but not their replies, are similarly colored to indicate agreement or disagreement. Users sometimes want to agree or disagree without leaving comments; currently this leaves a default comment that says "Type the reason why you oppose..."

\footnotetext{
${ }^{78}$ http://arg.umentum.com/
}

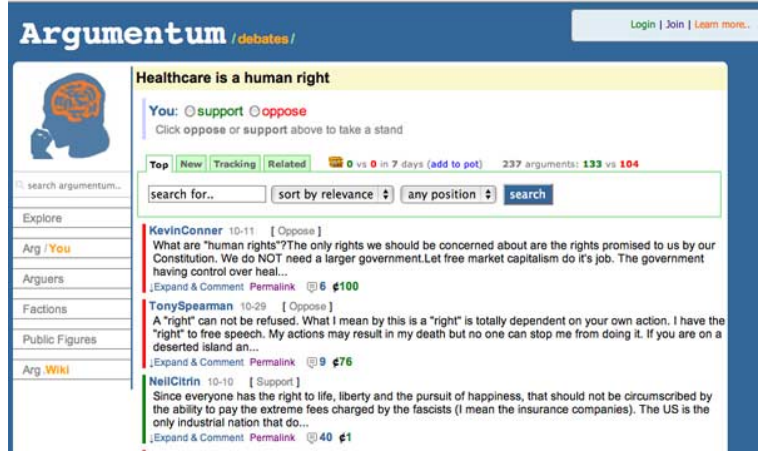

Fig. 26. In Argumentum, users can indicate support for an argument with money. The left-hand color bars indicate the supporting (green) and opposing (red) arguments. (Color figure online)

Argumentum's most unique feature is that users can put their " 2 cents" in literally: credibility, earned with good arguments, is measured in 'cents' and can be spent to influence a debate result. Users can also contribute arguments without starting from the Argumentum website, using bookmarklets ${ }^{79}$ or through Gmail and Facebook ${ }^{80}$. Further, loggers and publishers can also contribute using Argumentum buttons or widgets.

\section{A.7. Argumentations.com}

Argumentations ${ }^{81}$ serves analysts who want to develop arguments collaboratively. Arguments, which are classified as either claims or open-ended issues, can be added or edited; an example is shown in Fig. 27 on the facing page. To help suggest topics and build arguments, users can import news stories and extract statements (declarative sentences) from stories.

Argumentations offers several unique features. First, arguments - whether claims or open-ended issues - are evaluated depending on their type. Claims are evaluated with a truth value and confidence. Open-ended issues are evaluated based on Desirability, Importance, Volatility, Likelihood, and Confidence. Second, along with tag clouds, Argumentations uses 'tag spheres' (Fig. 28 on the next page). Further, arguments can be opened in Silverlight. Finally, they offer some interesting tutorials which display mindmaps ${ }^{82}$.

\footnotetext{
${ }^{79}$ http://arg.umentum.com/share

${ }^{80} \mathrm{http}: / /$ arg.umentum.com/wiki/more-ways-to-argue

${ }^{81} \mathrm{http}: / /$ www.argumentations.com/

${ }^{82} \mathrm{http}: / /$ www.argumentations.com/Argumentations/Help/ Tutorials/Tutorials.aspx
} 


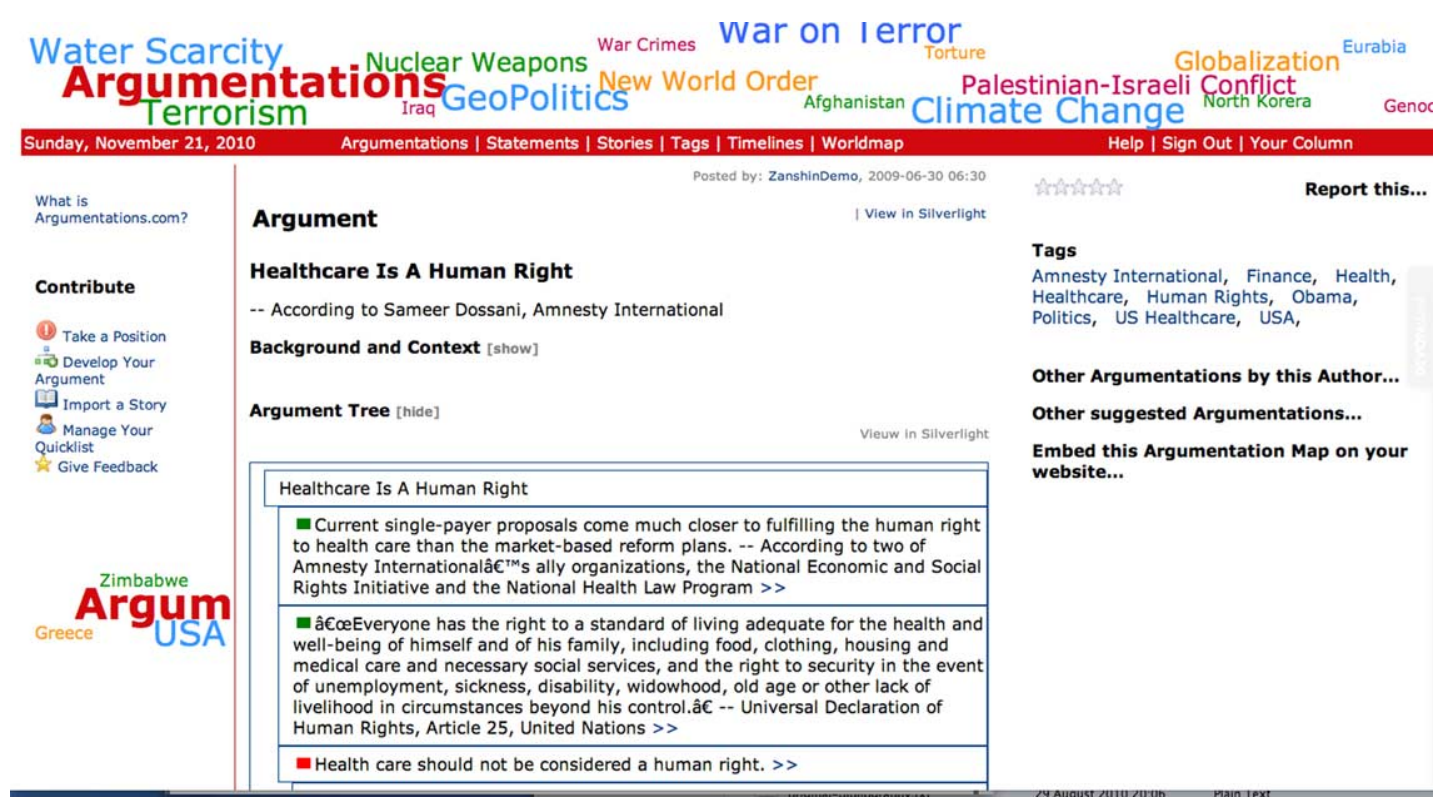

Fig. 27. In Argumentions, colored dots indicate the supporting (green) and opposing (red) arguments. (Color figure online)

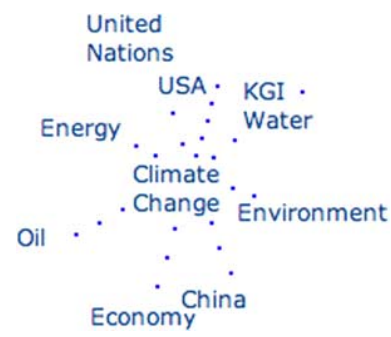

Fig. 28. The global warming 'tag sphere' from Argumentations. (Color figure online)

\section{A.8. Argunet}

Argunet [166] is a desktop tool ${ }^{83}$ coupled with an open source federation system for sharing argument maps. A public server, Argunet.org ${ }^{84}$, allows authors to make maps public or restrict viewing and/or editing to a specified group. Connecting to other servers is also possible; this focus on federation, makes Argunet unique.

Argunet also has other unique features. Argunet is a multi-lingual environment which records the language of the map. Maps published at Argunet.org, must be released under the CC-BY license. An extensive online manual provides instruction, and they promote embedding debates. Users also have significant control over the presentation of arguments, such as colors and de-

\footnotetext{
${ }^{83} \mathrm{http}$ ///www.argunet.org/editor/

${ }^{84} \mathrm{http}$ ///www.argunet.org/debates/
}

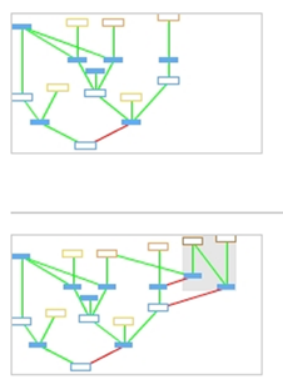

Phase 2
The substantiation that the fossilized plants 16
Edges: 17 lie at the top of the Greywacke triggered a Inferential Density: new anomaly. On the one hand, the fossil 1.13 criterion $(\mathrm{H} 1)$ seemed to imply that the top layers of the Greywacke are Carboniferous (Fossil-clock-argument). On the other hand, all British Carboniferous coal ...

Phase $3 \quad$ Nodes: 20 Murchison and his colleagues assembled Edges: 23 empirical evidence from all over Europe, Inferential Density: hoping to obtain clues about the correct 1.21 expedition to Russia in 1840 , they found.

(a)

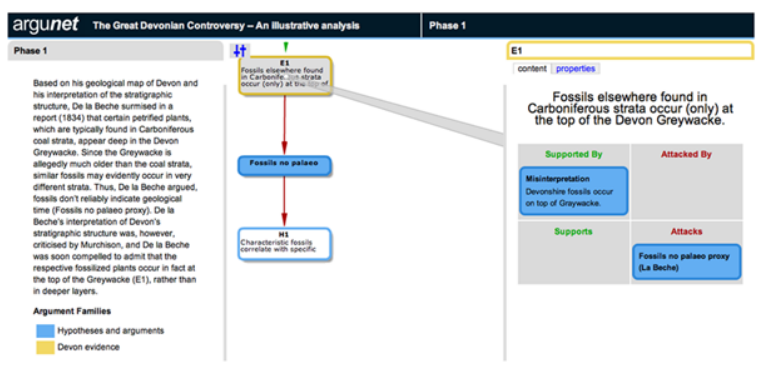

(b)

Fig. 29. Argunet can show an (a) overview of several related argument maps; and (b) in each individual map, nodes can be opened up to show arguments they support, attack, are supported by, and are attacked by. (Color figure online)

scriptions of different argument families. Related maps can be published in series, as shown in Fig. 29(a). In the argument map representation, each node can be opened up to reveal a matrix listing which other arguments support, attack, are supported by, and are at- 


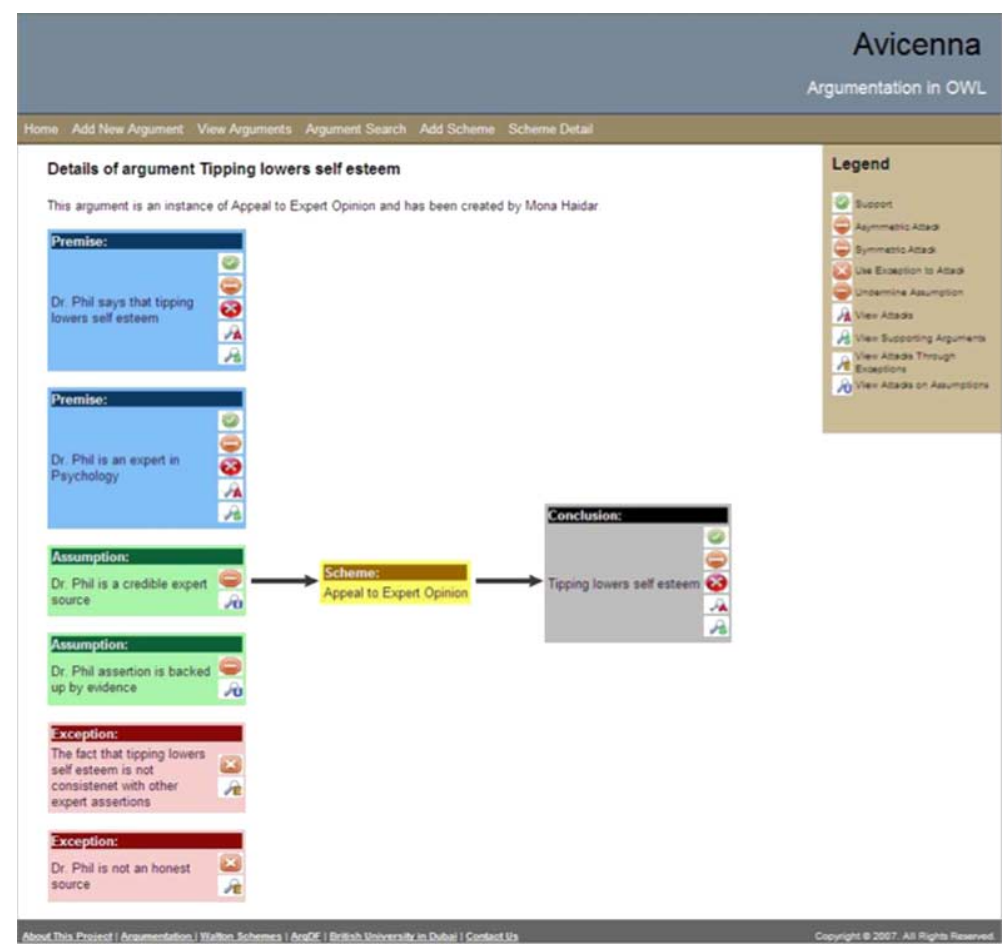

Fig. 30. Avicenna uses Walton's critical questions and argument schemes [145]. (Color figure online)

tacked by the given node (Fig. 29(b) on page 199). Argunet appears to support incremental formalization since arguments can be quickly sketched or reconstructed as premises and conclusions.

\section{A.9. Avicenna}

Rahwan and Banihashemi's OWL-based argumentation system Avicenna (Fig. 30) was demonstrated at COMMA 2008 [144] and recent descriptions and screenshots appear in [145]. Extending the work of ArgDF, Avicenna is a Web-based system using Jena [33], $\mathrm{ARQ}^{85}$, and Pellet [173]. Since OWL supports inference over transitive properties, Avicenna can support argument chaining, such as retrieving all arguments that directly or indirectly support a given conclusion. Avicenna is also used to infer the classification hierarchy of argument schemes: for example, an appeal to expert opinion is a specialization of an argument from position to know.

\section{A.10. bCisive online}

bCisive Online ${ }^{86}$ is an online argument mapping and spatial hypertext environment for real-time col-

\footnotetext{
${ }^{85} \mathrm{http} / / /$ jena.sourceforge.net/ARQ/

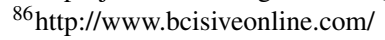

laboration and team problem-solving (Fig. 31(a) on page 201). Aimed at the business market and individual decision-makers, bCisive Online is a commercial product from AusThink, the makers of the Rationale desktop tool; the free option allows up to three users to collaborate, or users can upgrade with a monthly subscription fee. bCisive Online is unique in that it is intended for real-time use with audio conferencing. One person edits the map at a time, adding nodes and connections between nodes (Fig. 31(b) on page 201) while others can point with their cursor or request editing control. Maps can be embedded in blogs (which allows viewers to pan, zoom, hide and show parts of the map) or exported as PowerPoint. Snapshots can be saved as history items, to allow restoring to or reviewing a previous map.

\section{A.11. Belvedere}

Belvedere $^{87}$ is open source software for problembased collaborative learning. It provides multiple views, such as tables, graphs, and argument maps, of the same topic (Fig. 32 on the next page). It has been extensively investigated in studies of computersupported collaborative learning [179].

\footnotetext{
${ }^{87} \mathrm{http}: / /$ belvedere.sourceforge.net/
} 


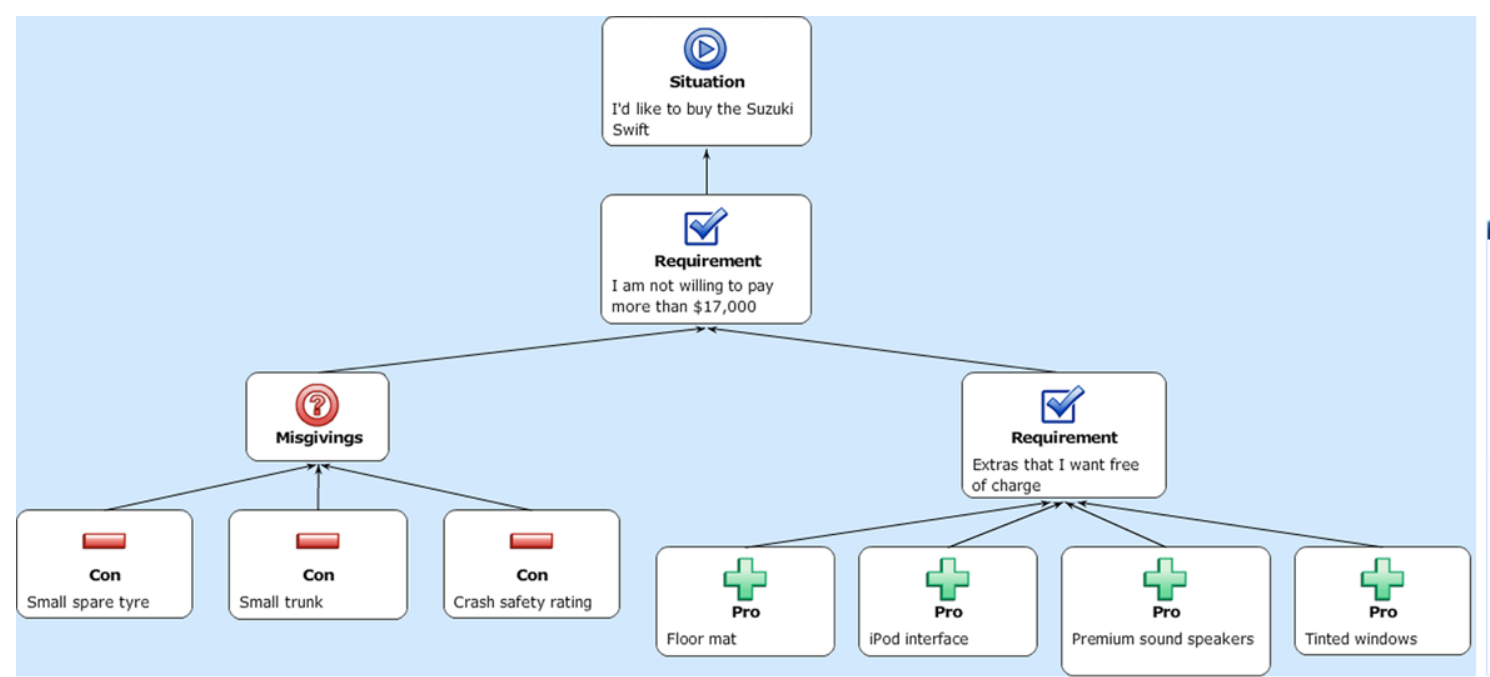

(a)

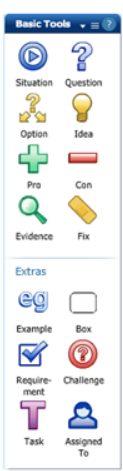

(b)

Fig. 31. (a) Collaborative maps for bCisive Online can be used for decision-making and requirements analysis. (b) bCisive Online's node types show the kinds of discussions that it facilitates. (Color figure online)

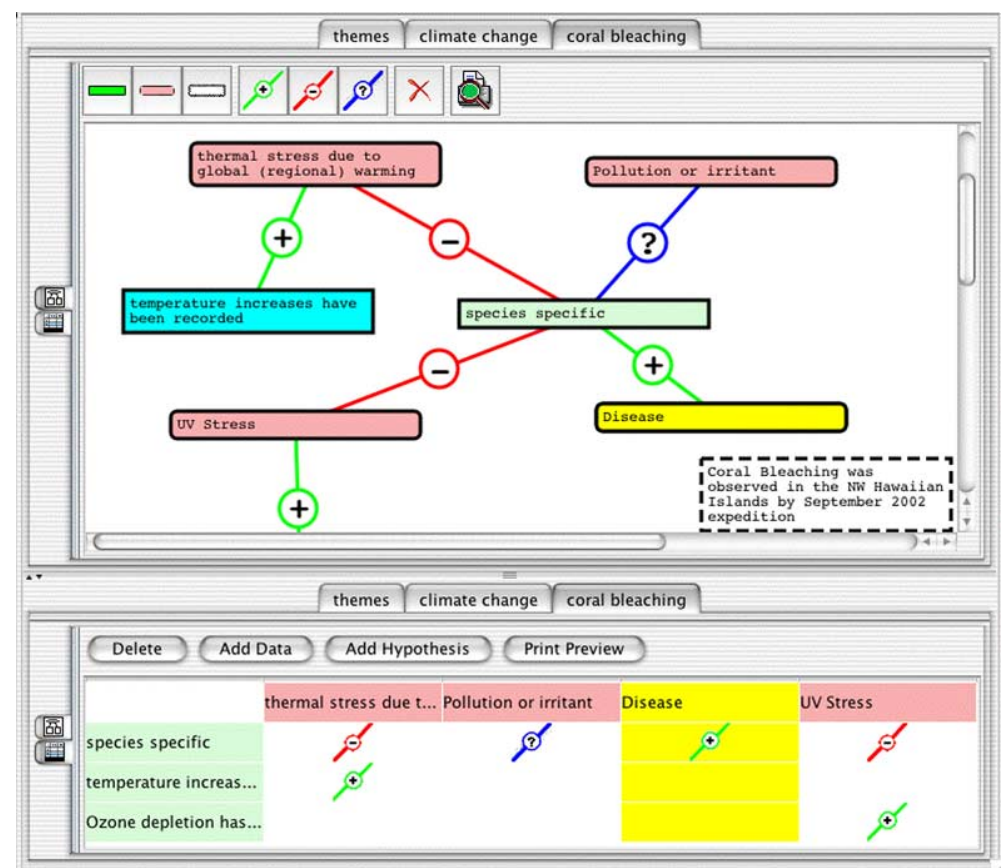

Fig. 32. Belvedere has both argument maps and tables to help organize evidence in collaborative learning. (Color figure online)

\section{A.12. Cabanac's annotation system}

Cabanac used a Java-based system ${ }^{88}$ to research social validation of the arguments in comments [30]. Users did not contribute new content to an ongoing public debate, but analyzed the argumentative status of

${ }^{88}$ http://www.irit.fr/ Guillaume.Cabanac/expe/ document comments. Uniquely, sliders were used to indicate the extent to which items were refuted, neutral, or confirmed (Fig. 33 on the following page). In effect, users were asked to synthesize the discussion. Aggregated information was not viewed by the users, but held by the experimenter. However, in principle, this approach could be used to promote collaborative sensemaking not just of annotations but also of debate. 


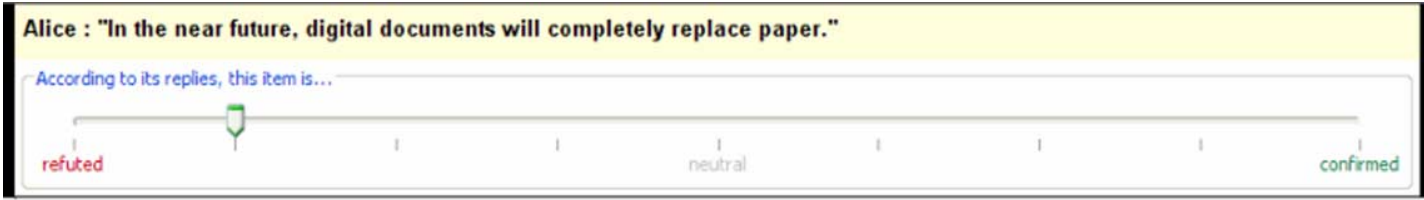

Bob : "Digital documents are not handy enough."

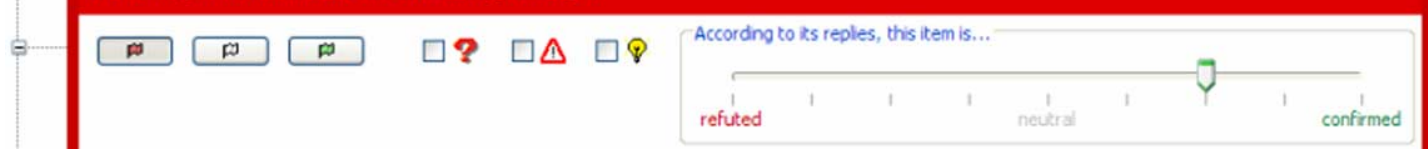

Fig. 33. Cabanac had users flag items (refuted, neutral, confirmed) and indicate their types (question, modification, example). (Color figure online)

\section{Proposals:}

+show help

Should developed countries provide
funding to help developing nations
address climate change?
Position
$\begin{aligned} & \text { Yes: Developed countries should } \\ & \text { provide funding. }\end{aligned}$
$\begin{aligned} & \text { Rich countries created the } \\ & \text { problem } \\ & \text { will be relatively small } \\ & \text { will not support such } \\ & \text { transfers }\end{aligned}$
po: Developing countries should their own way
Each nation should take
responsibility for itself
$L$
Without financing,
paid their way
emission reductions will
start later

\section{? Question \\ Should developed countries provide funding to help developing nations address climate change?}

A key issue that emerged at the Copenhagen climate talks was whether developing countries would provide financing to help developing nations defray the cost of emission reductions and adaptation. For more, see Financial transfers in climate negotiations

\section{References:}

1. Project Catalyst, Briefing Paper: Overall Financing Needs, December 2009 (4 page pdf file)

\section{$D$ tshow help Comments}

It is difficult to figure out exactly how much developed nations should compensate developing nations for adaptation and mitigation.

There is starting to be more research on the exact costs of damages to the economy from climate change, though.

Here's a paper on Temperatures and cyclones strongly associated with economic production in the Caribbean and Central America

http://www.pnas.org/content/107/35/15367.full

(a)

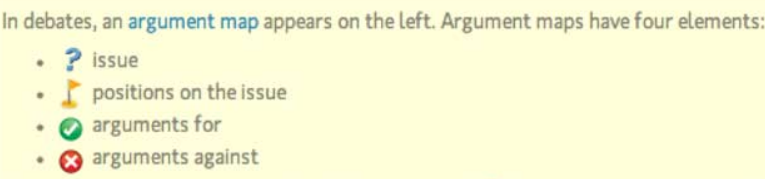

Click on any item to view more detail. You can vote $(\mathcal{V})$ on one position per issue. To add to the summary, log in and click the Advanced button. At the right, you may comment $(\square)$ on any item.See more.

Hide this message

(b)

Fig. 34. At Climate CoLab, (a) the positions tab shows an argument map which users can edit or comment on. (b) argument maps are introduced with contextual help. (Color figure online) 


\section{A.13. Climate CoLab}

The Climate $\mathrm{CoLab}^{89}$ is a deliberation platform under development at MIT, building on former projects such as the Deliberatorium and the ClimateCollabatorium $[80,97,98]$. The community runs an annual contest to gather proposals for mitigating global warming from the general public; once proposals are filtered by experts, everyone is invited to discuss the finalists.

Users deliberate in the Positions tab, which facilitates constructing an argument map, voting, and commenting on each of five key topics. Moderators are expected to review comments and add new ideas to the argument map; users can also add Pros, Cons, and Issues directly to an argument map. The Climate CoLab is unique for integrating argument maps into a larger debate, and for its moderator support, which allows users to benefit from argument maps without necessarily needing to understand how to edit them.

\section{A.14. Cohere}

Cohere is open source software for sensemaking which integrates annotation and argumentation for the general public [26,110]. At the Cohere website ${ }^{90}$, users can view and create maps, or import them from the Compendium desktop software. Maps consist of ideas, which users can add directly on the site (Fig. 35), draw from Cohere's global pool of public ideas, or clip via a Firefox plugin while browsing.

Cohere is unique for its integration with the Compendium desktop software, its incorporation of social bookmarking, and the ability to mark information as private, public, or shared with a group. Cohere also offers an $\mathrm{API}^{91}$.

\section{A.15. Competing Hypotheses}

Competing Hypotheses ${ }^{92}$ is open source analysis software based on the CIA methodology "Analysis of Competing Hypotheses" (ACH). The software supports breaking down information into hypotheses, evidence, and analysis, which are entered into a matrix as shown in Fig. 36(a) on page 204. The matrix can help visually indicate the most likely and least likely scenarios. ${ }^{93}$ Multiple analyses can be combined to provide a

\footnotetext{
${ }^{89} \mathrm{http}: / /$ climatecolab.org/

${ }^{90} \mathrm{http} / / /$ cohere.open.ac.uk/

${ }^{91} \mathrm{http}: / /$ cohere.open.ac.uk/help/code-doc/

${ }^{92} \mathrm{http}: / /$ competinghypotheses.org/

${ }^{93}$ More sophisticated $\mathrm{ACH}$-based software uses matrices as input to Bayesian probabilistic reasoning.
}

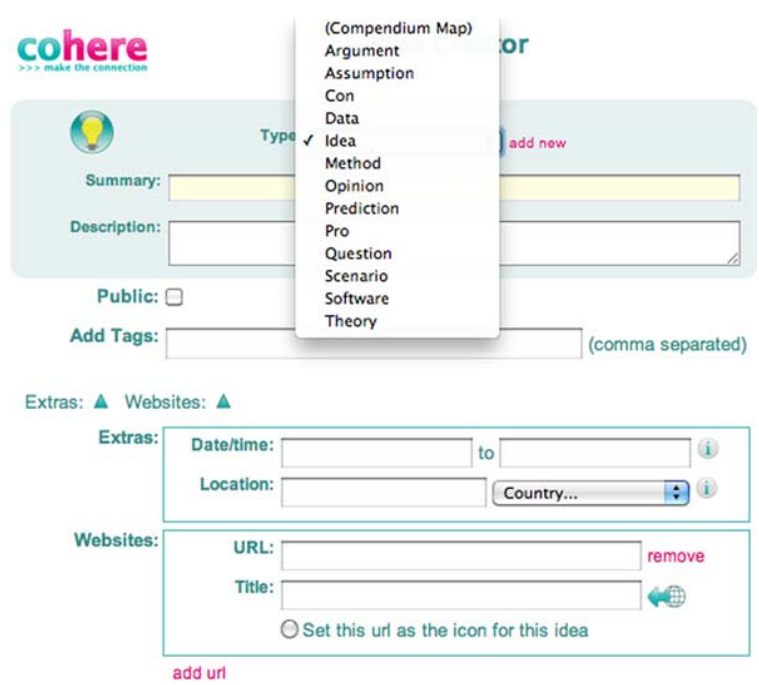

Fig. 35. Adding an idea to Cohere. (Color figure online)

group view (Fig. 36(b) on page 204), or compared pairwise. Competing Hypotheses has persistent chat (essentially a comment thread) for the entire project as well as message boards for each hypothesis, evidence item, and evidence-hypothesis pair. We excluded earlier $\mathrm{ACH}$ implementations such as PARC $\mathrm{ACH}^{94}$. Unlike these systems, Competing Hypotheses has a testing server ${ }^{95}$ which allows online collaboration. It is unique for its visualization structure and its use of both individual and group information.

\section{A.16. ConsiderIt}

ConsiderIt $^{96}$ [102] is a new open source deliberation platform under development at the University of Washington. It powers the Living Voters' Guide ${ }^{97}$, a deliberation and voter-information platform for Washington State voters.

What is unique is the possibility to drill down to understand other voters' perspectives. In addition to seeing pros and cons on an issue from all voters, regardless of their stance, (Fig. 37(a) on page 204), the Living Voters' Guide can show the key points for a particular group of voters (Fig. 37(b) on page 204), such as those undecided on the issue or strongly supporting

\footnotetext{
${ }^{94} \mathrm{http}: / / \mathrm{www} 2$. parc.com/istl/projects/ach/ach.html

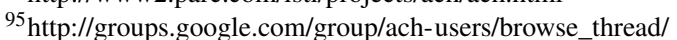
thread/d87a5ec4df8be6c0

${ }^{96} \mathrm{http}$ ://www.livingvotersguide.org/considerit

${ }^{97} \mathrm{http}: / / w w w . l i v i n g v o t e r s g u i d e . o r g /$
} 


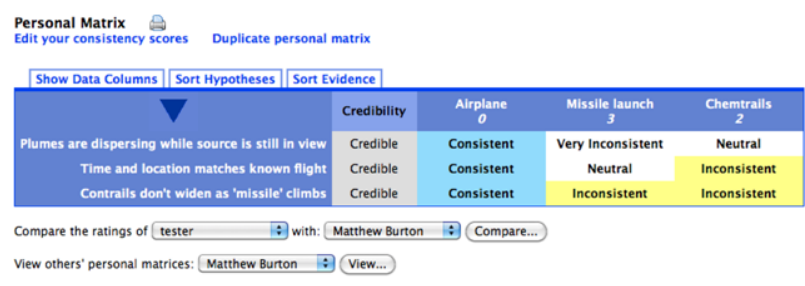

(a)

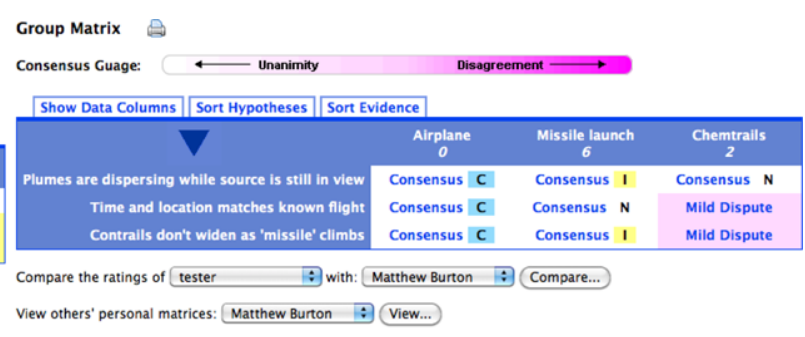

(b)

Fig. 36. In Competing Hypotheses, (a) each individual's analysis is represented in a consistency matrix; (b) multiple analyses can be combined to create a group matrix. In the group view, darker shades of purple indicate more disagreement. (Color figure online)

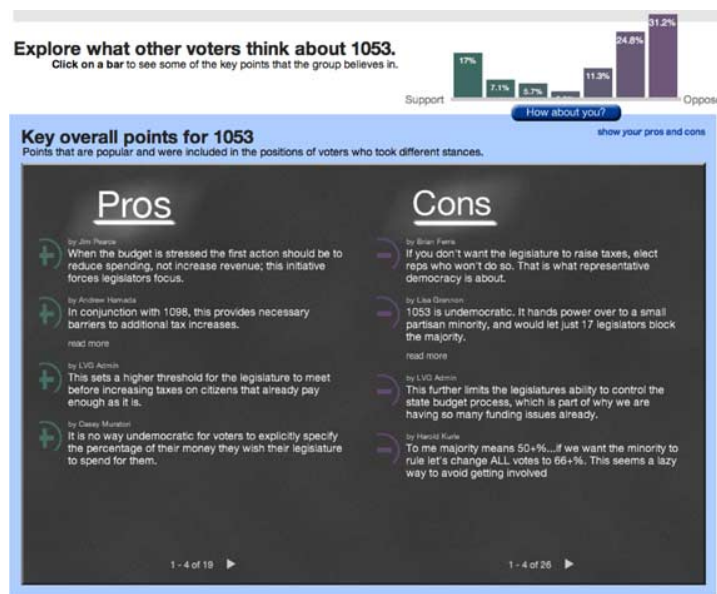

(a)

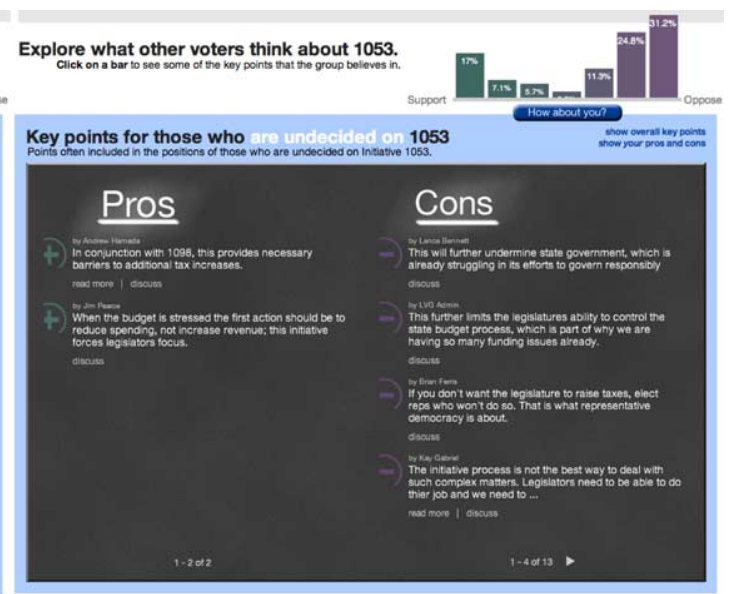

(b)

Fig. 37. The Living Voters' Guide compiles pro and con lists on each issue. They give (a) an overview of what all voters think about the issue; as well as (b) the key points for undecided voters. (Color figure online)

it. This can help users understand what makes an issue controversial. Users indicate how they feel about an issue before and after reading an argument (deliberative polling), which could also be used to find the most convincing arguments.

\section{A.17. ConvinceMe}

ConvinceMe $\mathrm{e}^{98}$ is a competitive debating environment which uses a point scheme and user rankings to motivate contributions to several types of debates. In the King of the Hill game, the most popular choice (and the debater who suggested it) wins. Battles are oneon-one debates between two users, who add arguments and evidence in hopes of getting readers' votes; the debate ends when one side gets a pre-agreed number of votes. Open debates (Fig. 38) are ongoing and accept pro or con arguments from any registered user, as well

\footnotetext{
${ }^{98}$ http://www.convinceme.net/
}

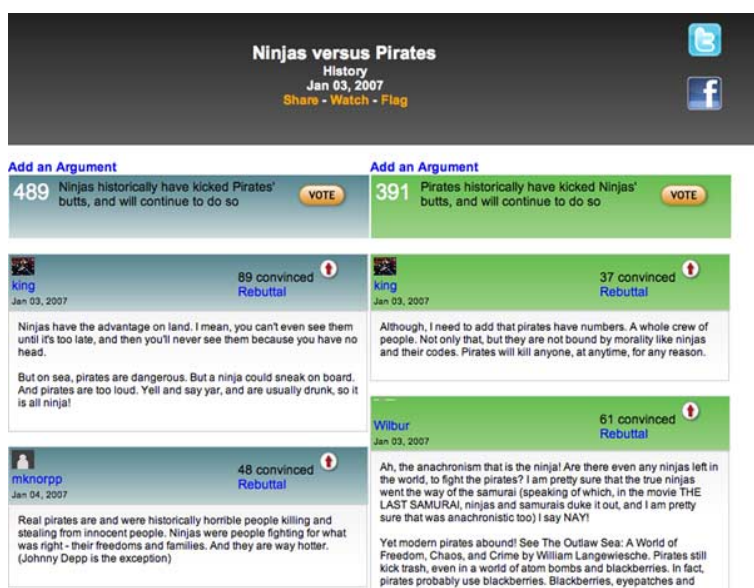

Fig. 38. In ConvinceMe's Open Debates, users can vote for an argument that convinced them. (Color figure online)

as rebuttals to existing arguments; users convinced by an argument vote for it. These various types of debate games make ConvinceMe unique. 


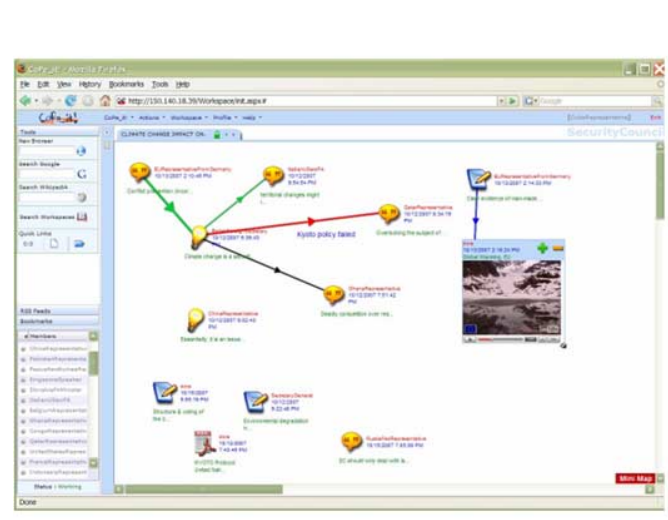

(a)

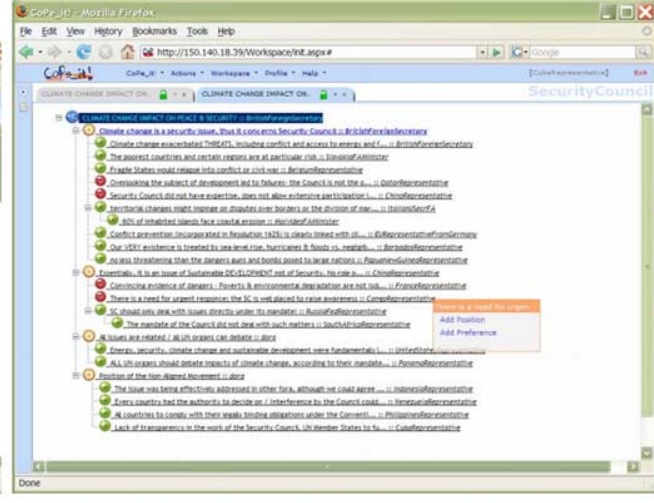

(b)

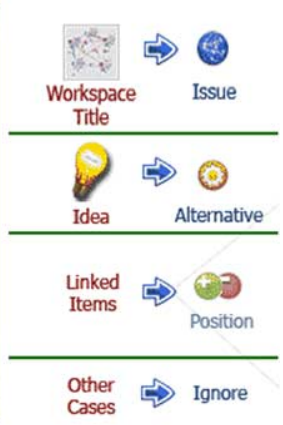

(c)

Fig. 39. CoPe_it! has (a) an informal spatial hypertext view; and (b) a formalized view, created by (c) automatically transforming items. (Color figure online)

\section{A.18. CoPe_it!}

CoPe_it! ${ }^{99}$ [187] is a spatial hypertext environment for collaboration, aimed at the learning and e-government domains. Users can form groups to share maps, but communicate only through email on the site. Maps can be imported from Compendium, and entire discussions from external webforums in phpNuke format can be imported using a URL.

One unique aspect of in CoPe_it! is its approach to incremental formalization. CoPe_it! transforms the user-created informal spatial hypertext view (Fig. 39(a)) into an issue chart Fig. 39(b) according to rules shown in Fig. 39(c). Users can also customize the transformation rules.

\section{A.19. CreateDebate}

CreateDebate $^{100}$ is a social debate community, aimed at the general public as well as primary and secondary school classes ${ }^{101}$. The highest-rated arguments are shown at the top, based on user votes (and ignoring the down votes), which are also used to determine a point score for the user. They offer bookmarklets and promote JavaScript buttons to webmasters $^{102}$. Some unique features are that the debate moderator can add a 'Topic Research' section with RSS feeds from other sites, and that, in addition to pro/con debates, CreateDebate has Perspective debates, which generally have more than two sides, are scored based

\footnotetext{
${ }^{99}$ http://copeit.cti.gr/

${ }^{100} \mathrm{http}: / / \mathrm{www}$. createdebate.com/

${ }^{101} \mathrm{http}: / / \mathrm{www} . c r e a t e d e b a t e . c o m / a b o u t / s i t e s / s c h o o l$

${ }^{102} \mathrm{http}: / /$ www.createdebate.com/share/buttons
}

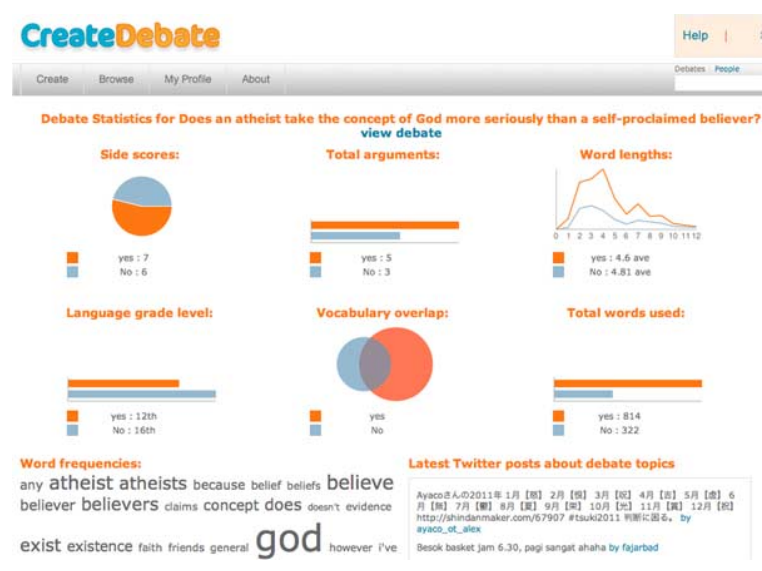

Fig. 40. At CreateDebate, users add and comment on pro and con arguments. (Color figure online)

on user-applied tags. A wordcloud and various statistics (Fig. 40), including the language grade level, average word lengths, and vocabulary overlap are calculated for each debate.

\section{A.20. Debate.org}

Debate.org ${ }^{103}$ is a social networking site for debate lovers. Debates take place between two members and have four cycles: the challenge period, debating period, voting period, and post voting period. The debating period consists of 1-5 time-limited rounds in which debaters post arguments. While comments can be added at any time, votes are only accepted during the voting period. Voting involves choosing one of the debators (or 'tied') for each of the fol-

${ }^{103}$ http://debate.org/ 


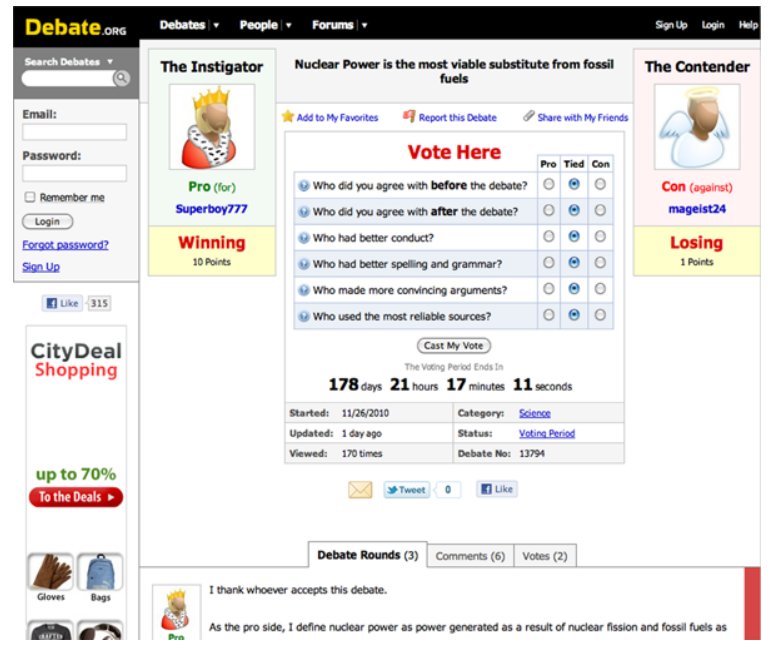

Fig. 41. Debate.org is a social networking site promoting debate. (Color figure online)

lowing six questions: (1) Agreed with before the debate: (worth 0 points) (2) Agreed with after the debate: (worth 0 points) (3) Who had better conduct: (worth 1 point) (4) Had better spelling and grammar: (worth 1 point) (5) Made more convincing arguments: (worth 3 points) (6) Used the most reliable sources: (worth 2 points). Points are awarded, with the most importance given to using reliable sources and making convincing arguments.

Another unique feature is Debate.org's focus on user profiles, where various user details are displayed including information such as income, location, ideology, gender, president, religion, and who they are interested in and looking for. These can be used to search for people with particular profile attributes, and aggregate user demographics ${ }^{104}$ are also available. Debate.org also determines the percentage to which other members agree with you on "the big issues" (cultural, religious, and political hot topics). Individual members are also ranked by their percentile, based on the outcomes of previous debates.

\section{A.21. Debategraph}

Debategraph $^{105}$ [115] is a wiki debate visualization tool which has been adopted for use at the Kyoto climate change summit and is being tested by EU projects such as WAVE ${ }^{106}$. Debategraph offers several visual-

\footnotetext{
${ }^{104} \mathrm{http}: / / w w w . d e b a t e . o r g / a b o u t / d e m o g r a p h i c s /$

${ }^{105} \mathrm{http}: / /$ debategraph.org/

${ }^{106} \mathrm{http} / / /$ www.wave-project.eu/
}

izations, including the Debate Explorer view shown in Fig. 42(a) on page 207 and a text-based outline shown in Fig. 42(b) on page 207. Visualizations can be embedded in other websites, and Debategraph encourages users to add links to related webpages within graphs.

\section{A.22. Debatepedia}

Debatepedia" ${ }^{107}$ bills itself as the "the Wikipedia of pros and cons". Sponsored by the International Debate Education Association, Debatepedia is a collaborative community effort to summarize arguments. Each argument page provides an overview, then a list of issues, with pros and cons supported by news articles and similar sources. It provides an intuitive editing environment, where users can edit just the relevant section, such as the pro or con for a topic. Debatepedia is unique for providing an easily-editable wiki of pros and cons.

\section{A.23. Debatewise}

On Debatewise ${ }^{108}$, everyone can collaborate in creating the strongest case both for and against a given issue. As part of a partnership with the International Debate Education Association (iDebate), they provide links to Debatepedia and iDebate's reference site Debatabase ${ }^{109}$. Karma, teams, and lists of recent participants and new editors help motivate participation.

There are several unique features. The site makes it easy to get involved by providing suggestions of 5-minute, 20-minute and 1-hour tasks and showing "7 things you should have an opinion on" in rotating images on the homepage. Edit histories are available for each pro and con point. Debates are structured as adjudicated debates between two teams; other users can make comments, vote, and subscribe to debates.

\section{A.24. DiscourseDB}

DiscourseDB ${ }^{110}$ is used to collaboratively collect policy-related commentary. Opinion pieces (Fig. 44(a) on page 208) are collected from notable sources, newspapers and websites with at least 50,000 circula-

\footnotetext{
${ }^{107}$ http://debatepedia.idebate.org/

${ }^{108} \mathrm{http}: / /$ debatewise.org/

${ }^{109} \mathrm{http}: / /$ www.idebate.org/debatabase/intro.php

${ }^{110}$ http://discoursedb.org/
} 


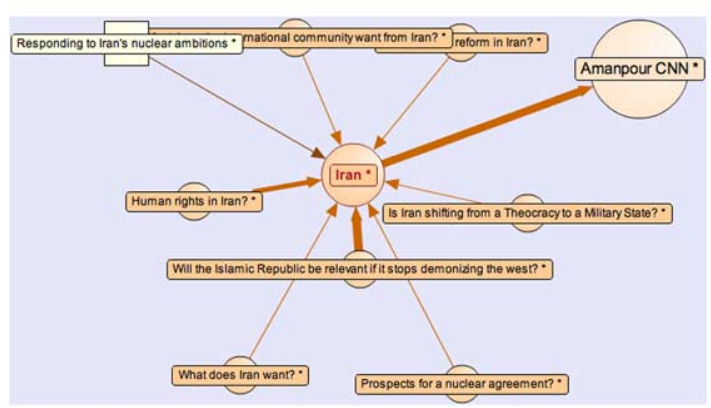

(a)

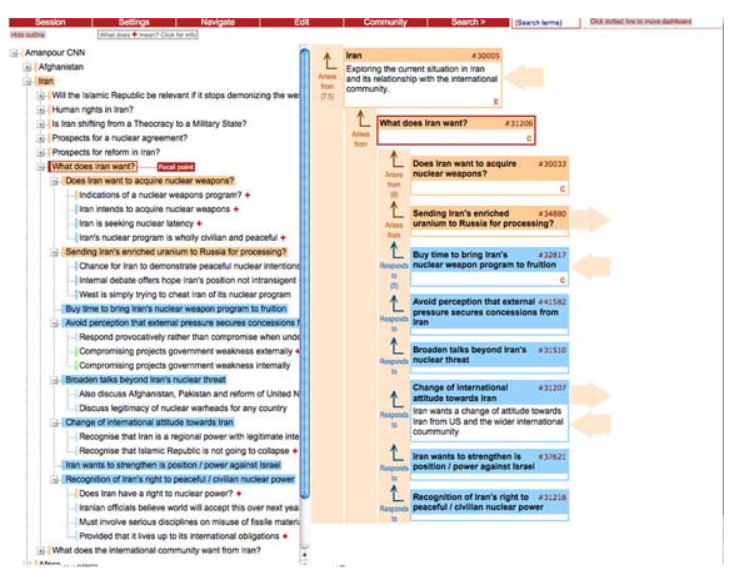

(b)

Fig. 42. Debategraph for CNN's Amanpour TV shown in (a) Debate Explore view; (b) text view. (Color figure online)

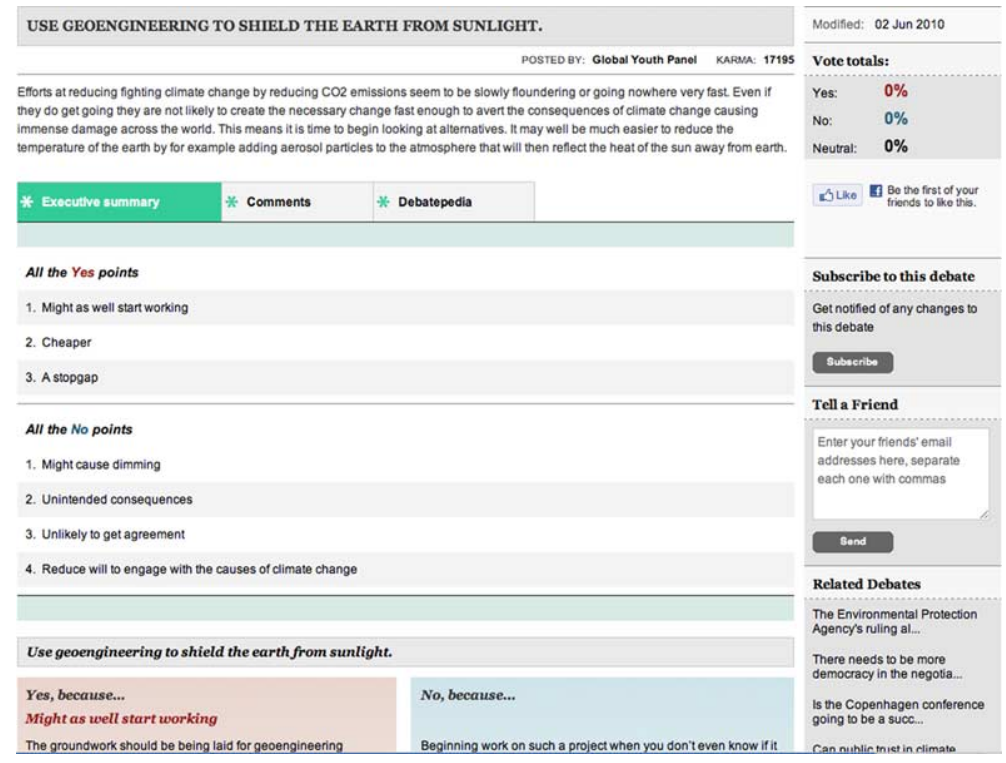

Fig. 43. Debatewise offers an executive summary, followed by a detailed pro/con debate. (Color figure online)

tion/unique visitors per month. Users categorize these opinion pieces, selecting a quote, indicating the topic and position, along with whether the author's argument is for, against, or mixed on the position.

DiscourseDB uses Semantic MediaWiki [104] with the SemanticForms ${ }^{111}$ extension. This makes it possible to list all commentary written by particular person, published in a particular venue, and so forth.

Further, since items indicate the position they take on a topic, DiscourseDB can list all commentary for

${ }^{111}$ http://www.mediawiki.org/wiki/Extension:Semantic_Forms or against a given position as shown in Fig. 44(b) on page 208. When a topic has multiple positions (e.g. Darfur $^{112}$ ), DiscourseDB is especially helpful in summarizing the discussion.

\section{A.25. Dispute Finder}

Dispute Finder ${ }^{113}[60,61]$ is a browser extension that alerts users when information they read is disputed, based on a database of disputed claims. This

\footnotetext{
${ }^{112}$ http://discoursedb.org/wiki/Darfur_conflict

${ }^{113} \mathrm{http}: / /$ ennals.org/rob/disputefinder.html
} 


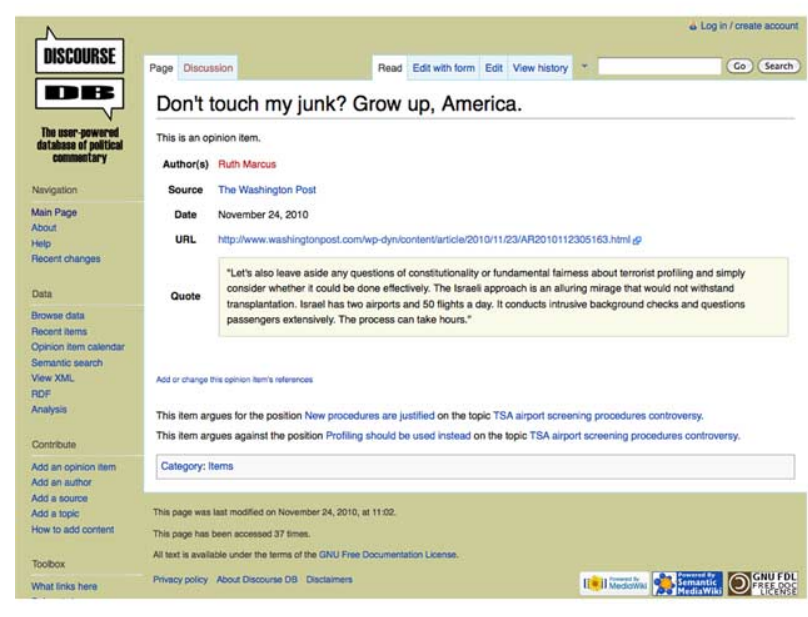

(a)

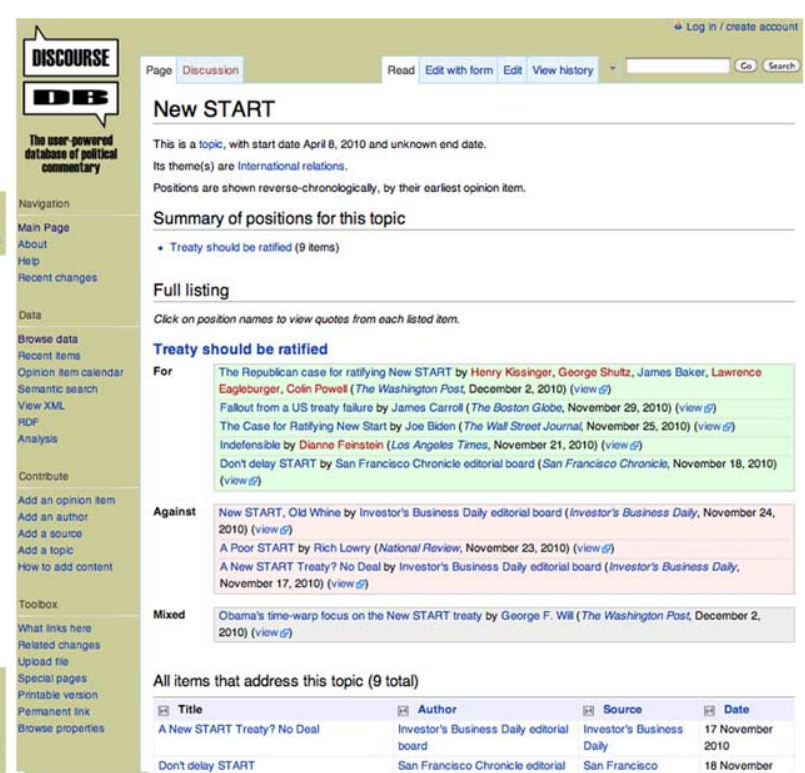

(b)

Fig. 44. In DiscourseDB, (a) users catalog opinion pieces; (b) this generates an overview of the positions for, against, and mixed on a topic. (Color figure online)

\section{Kind of message:}

(If this node is a Message.)
$\odot$ it None $\bigcirc$ Idea
? Question $\bigcirc \rightarrow$ More $\bigcirc$ Angry

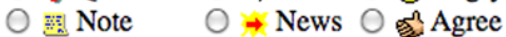
$\bigcirc \triangle$ Warning $\bigcirc \bigcirc \mathrm{Ok}$
$\bigcirc \odot$ Feedback $\bigcirc \odot$ Sad

(a)

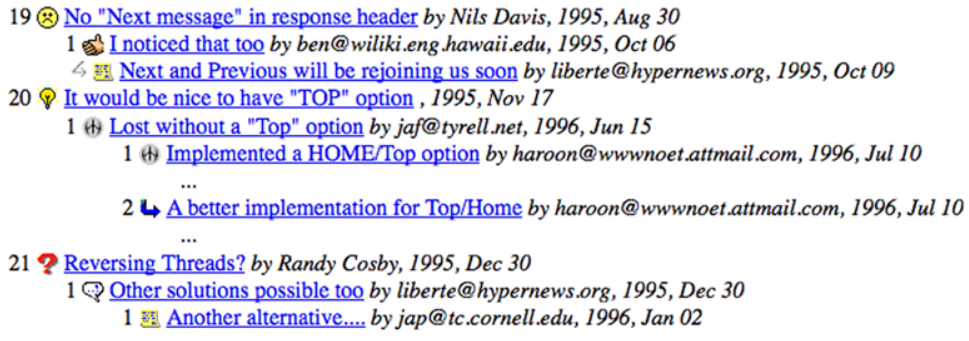

(b)

Fig. 45. (a) Users are asked to specify their message type, using this Hypernews taxonomy; (b) Part of a Hypernews discussion thread. (Color figure online)

database was created by asking activists (who are interested in informing or convincing others) to indicate disputed claims manually, and then extended algorithmically. While the Dispute Finder plugin remains available ${ }^{114}$, it notes that the project has ended; unfortunately, the plugin no longer highlights phrases such as the "abortion reduces crime" phrase used in paper examples.

\section{A.26. Hypernews}

Hypernews ${ }^{115}$ [18] is a general purpose Web forum, inspired by Usenet news. Its use of message types dis-

\footnotetext{
${ }^{114}$ http://addons.mozilla.org/en-US/firefox/addon/11712/

${ }^{115} \mathrm{http}: / /$ www.hypernews.org/HyperNews/get/hypernews/ reading.html
}

tinguishes HyperNews from other forums. Users are asked to indicate what kind of message they are posting (None, Question, Note, Warning, Feedback, Idea, More, News, Ok, Sad, Angry, Agree, Disagree) as shown in Fig. 45(a); the message type is then displayed as an icon in the forum's thread view (Fig. 45(b)).

\section{A.27. LivingVote}

At Living Vote ${ }^{116}$, the general public can discuss pro and con arguments of issues, creating argument maps, as shown in Fig. 46 on the next page. A tree view provides a coherent view of the argument, which can be drilled down, where arguments and their counter-

\footnotetext{
${ }^{116} \mathrm{http}: / /$ www.LivingVote.org/
} 


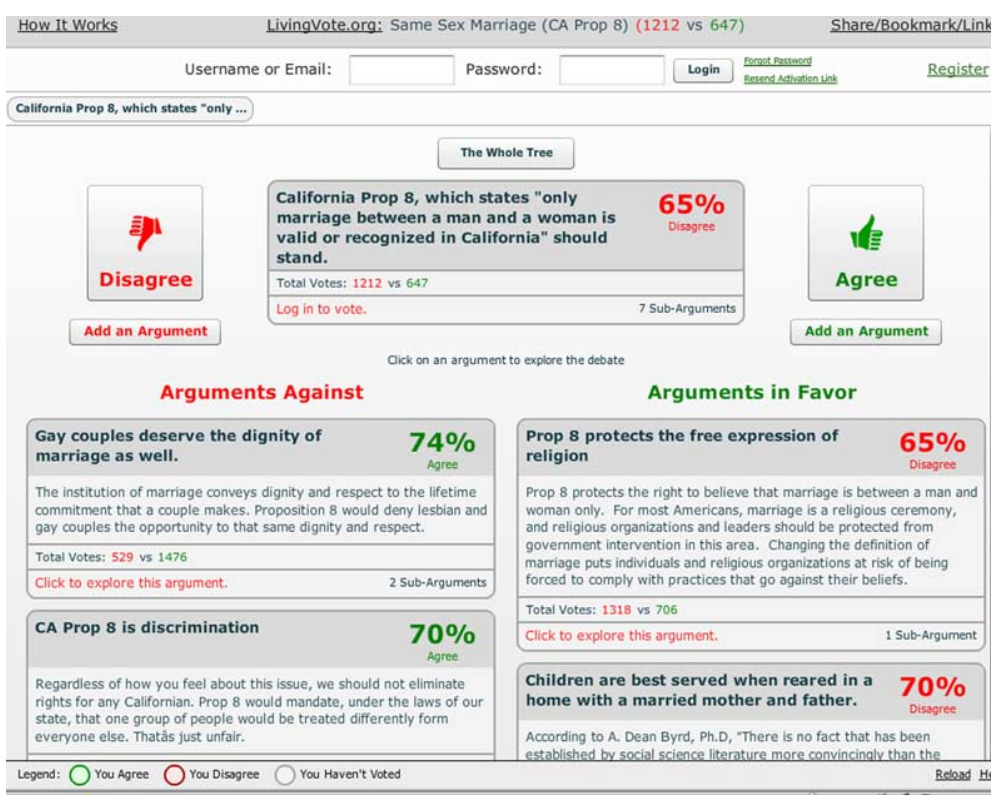

Fig. 46. At LivingVote, the weight given to a user's votes increases as they read and vote on more arguments. (Color figure online)

arguments are presented side-by-side. Users can add arguments, and voting colors the nodes according to whether you agree (green), disagree (red), or haven't voted (white).

Living Vote is unique in the way that it handles and uses votes. To vote, users must answer questions designed to test whether they've read the arguments. LivingVote also prunes unhelpful arguments and aims to provide a "complete, persistent, constantly changing and up-to-date record" of everyone's opinions and the most convincing arguments.

\section{A.28. Opinion Space}

Opinion Space is software developed by UC Berkeley's Center for New Media "designed to collect and visualize user opinions" on a variety of topics [63]. The U.S. Department of State is using Opinion Space $^{117}$ to aggregate opinions about foreign policy and create a "virtual town hall" as shown in Fig. 47.

Opinion Space is unique in its use of deliberative polling and visualization. With deliberative polling, participants are polled both before and after deliberation, to better understand how public opinion can change based on increased understanding of the issues. Users move sliders to express their opinions on five issues. The system then maps the user's opinion, using

${ }^{117}$ http://www.state.gov/opinionspace/

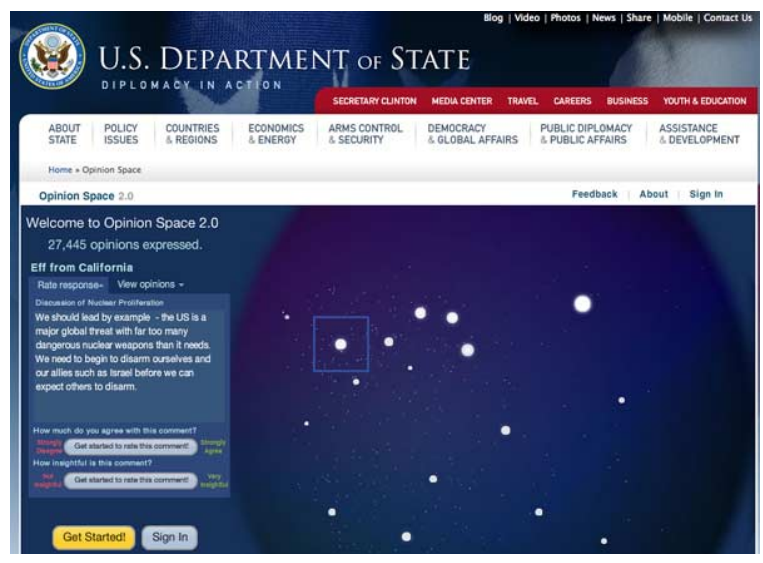

Fig. 47. Opinion Space maps comments in a constellation view. (Color figure online)

principal component analysis, to show the user where they stand. Each point in the visualization represents a perspective; larger points represent more popular perspectives. Users can also view and rate others' comments (Fig. 48 on the following page). Ratings can be used to choose the most informative comments for display.

\section{A.29. Online Visualisation of Arguments (OVA)}

Online Visualisation of Arguments ${ }^{118}$ (OVA) is an online argument analysis and mapping environment

${ }^{118} \mathrm{http}$ //ova.computing.dundee.ac.uk 


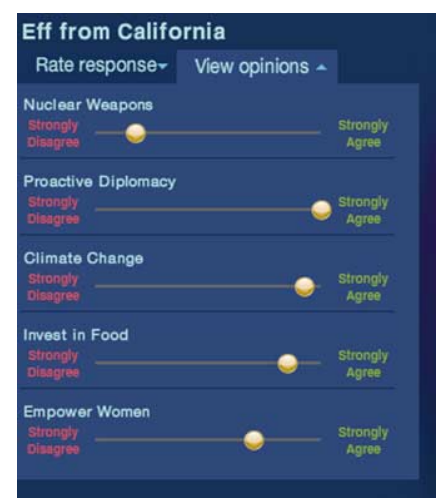

Fig. 48. Opinion Space uses sliders to collect and display users' opinions on five issues. (Color figure online)

[175] which exports AIF. In OVA, web pages can be displayed adjacent to an argument mapping canvas, helping analysts create a graphical representation of the arguments in online forums or news stories. The resulting argument maps can show the relationships between premises (supporting or attacking) as well as the participants responsible for each point of view. In addition to AIF, users can export JPEG and SVG images of the argument.

OVA is part of a pipeline of argumentation tools [174] which starts to bridge the gap between humanoriented argumentation tools and calculation-based agent argumentation. Mixed initiative discussions are enabled by the argument maps created by OVA or any other AIF-based tool. Thus, instead of representing one's point of view countless times in a forum or FAQ, it would be possible to delegate these conversations to a machine agent using an underlying argument map, as prototypes like MAgtALO ${ }^{119}$ [155,202] and the Google Wave discussion bot Arvina [175] show.

\section{A.30. Parmenides}

Parmenides $^{120}$ [35-37] is a structured survey tool for gathering public opinion on a proposal. Based on argument schemes and critical questions from argumentation theory, Parmenides can pinpoint the source of the disagreement, by having participants respond to a series of questions. In a Parmenides debates, participants are first asked to agree or disagree with a position on a question such as "Should lap-

\footnotetext{
${ }^{119} \mathrm{http}: / /$ www.arg.dundee.ac.uk/?page_id=61

${ }^{120}$ http://cgi.csc.liv.ac.uk/ parmenides/
}

tops be banned in lecture theatres?" (Fig. 49(a) on page 211). Those who disagree are stepped through several screens (such as Fig. 49(b) on page 211) of yes/no questions to determine the source of the disagreement. Limited free text boxes allow users to add further information. At the end of the survey, users are offered the choice of submitting an alternative proposal, and are shown the answers they chose. Administrators can then analyze the group's responses, which are displayed in graphical argumentation frameworks [52]. A greater understanding of the most popular reasons for disagreement could support further discussion and debate about the key issues.

\section{A.31. PDOnline}

SWAN/SIOC is itself used in PDOnline ${ }^{121}$, an online community for scientists, funders, and medical professionals working in Parkinson's disease science, which is funded by the Michael J. Fox Foundation [50].

Figure 50 shows a PDOnline discussion about a recently-published paper and indicates how the topic fits into the "PD Guide" taxonomy of research and communication topics. The discussion links both forward to responses and related contributions and back to a thread on Papers of the Week (itself contained within a Research Question board). Members' full names, credentials, and institutional affiliations are listed, with links to user profiles and institutions. Members' profiles link to their publications, and throughout the site explicit references to the literature are given. It is unique in that it uses scientific argumentation.

\section{A.32. REASON}

REASON - Rapid Evidence Aggregation Supporting Optimal Negotiation $[89,90]$ - is a Java applet for group deliberation, used to arrive at a consensus decision. Drawing from decision theory, group-decision support systems, and argumentation, REASON is intended to improve information pooling. An argument map is used to organize group evidence shared during the decision-making process; further, in an adaptive version of REASON, aggregate weights expressing the group's view of each alternative are displayed. Uniquely, arguments start as threaded discussions in

\footnotetext{
${ }^{121}$ http://www.pdonlineresearch.org/
} 


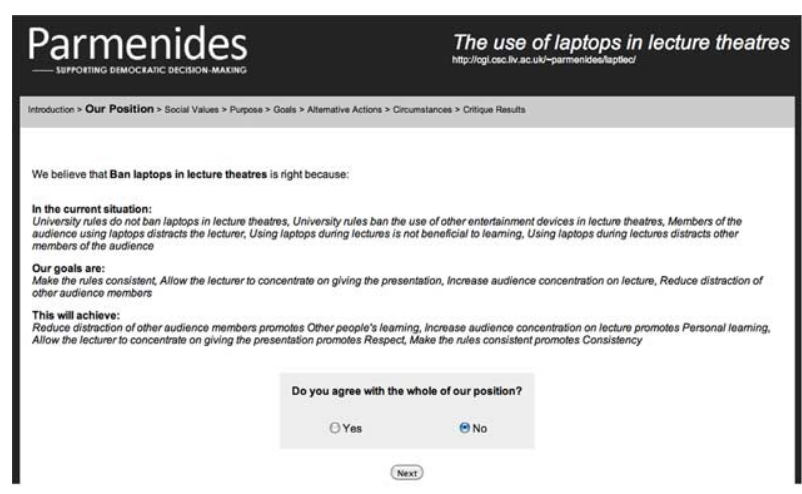

(a)

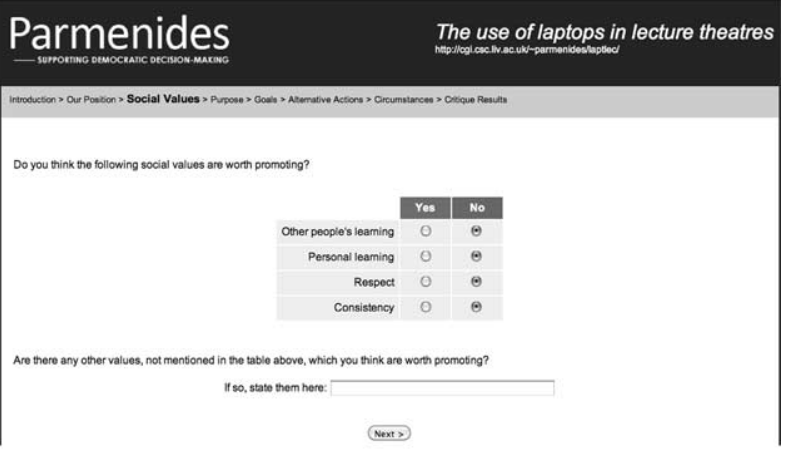

(b)

Fig. 49. (a) In Parmenides, participants are asked to agree or disagree with a starting position. (b) Next Parmenides steps participants through a series of yes/no questions to pinpoint the source of their disagreement. (Color figure online)

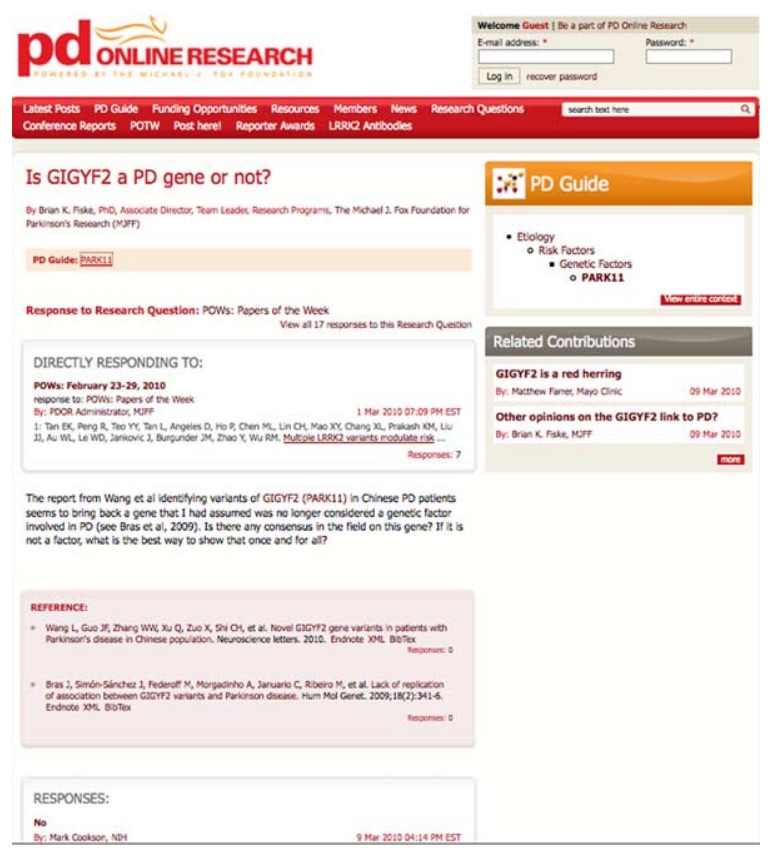

Fig. 50. Part of an argumentative discussion at PDOnline. (Color figure online)

REASON, and are colored based on whether they agree (blue) or disagree (yellow) with their parent in the thread.

\section{A.33. Riled Up!}

Riled Up! ${ }^{122}$ (Fig. 51(a) on page 212) is a debatecentered site which motivates participation with a point-based authority system. Aimed at people who

\footnotetext{
${ }^{122} \mathrm{http}: / /$ riledup.com/
}

enjoy debate, Riled Up!'s tagline is "Like Raising Cain? So Do We." Users can add debates, arguments, and comments, and vote for others' arguments, as well as add friends and enemies.

Riled Up! is unique in its comment system - users can respond with positive (green), neutral (grey), or negative (red) comments, as shown in Fig. 51(b) on page 212. In addition to a standard layout, a contributor view gives an overview of the arguments but not the comments.

\section{A.34. SEAS}

SRI International's SEAS ${ }^{123}[113,114]$ is a templatebased structured argumentation tool originally designed for collaborative intelligence analysis. It has since been tested in other domains such as by IRS tax auditors and in a simulated public health emergency. SEAS's most unique feature is its emphasis on templating; users can author templates which provide transferrable notions of how to make an argument, and specify authorized coeditors. Figure 52 shows one question from such a template. These templates, which are in essence domain-specific argument schemes, allow non-experts to make sound reasoning. SEAS automatically answers some questions based on earlier responses. The developers report that a threat-assessment template originally developed by U.S. intelligence analysts was successfully applied by non-experts in their laboratory. SEAS visualization features are also considerable: to visualize multiple dimensions, SEAS uses starburst, constellation,

\footnotetext{
${ }^{123}$ http://www.ai.sri.com/ seas/
} 


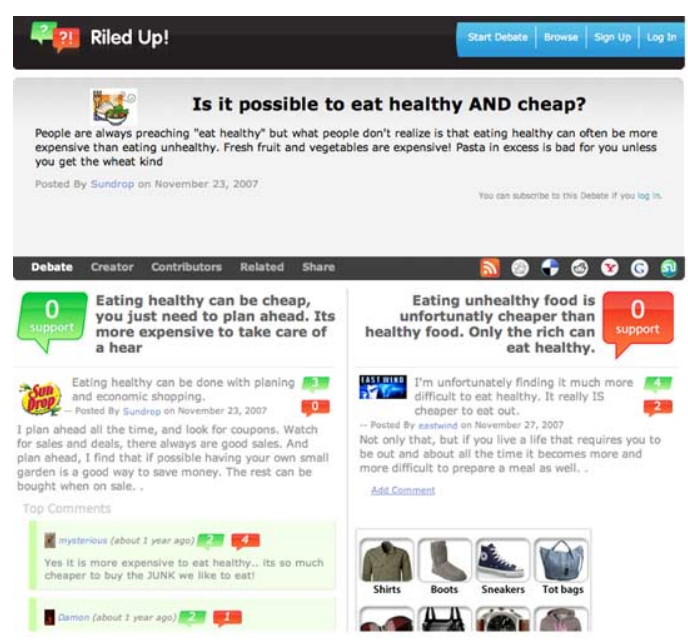

(a)

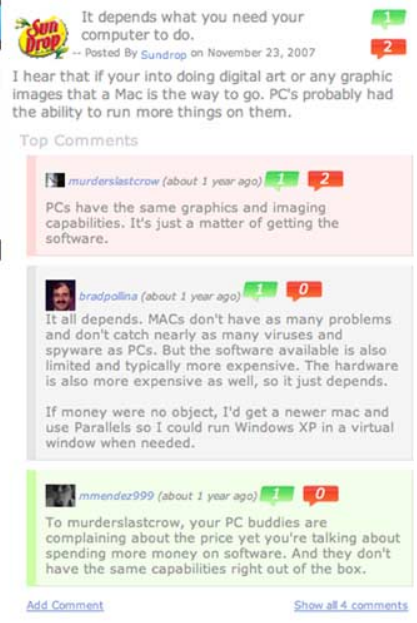

(b)

Fig. 51. RiledUp (a) debates allow structured discussion on a topic; and (b) readers can respond with positive (green), neutral (grey), or negative (red) comments. (Color figure online)

and table views. SRI International runs a SEAS server with paid accounts and SEAS server software is available.

\section{A.35. Trellis software}

The argument analysis system Trellis ${ }^{124}[42,43$, 70] was built on Semantic Web technologies, including the Semantic Annotation Vocabulary Section 7.7, page 178. Trellis, inspired by intelligence analysis, began as a credibility and analysis system to help structure decisions, for example to construct a family geneology based on contradictory information [70].

Originally, Trellis was designed to help capture argumentation, grounded in documents, whose reliability the user rated, and from which the user extracted statements; although users did not work directly with the underlying ontology, arguments could be exported into XML, RDF, DAML, and OWL. In addition to the original version, now called Rich Trells, two other modes, Tree and Table Trellis, described in [43], are now supported, for incremental formalization.

In Rich Trellis, statements are given likelihoodqualifiers such 'surprise' (indicating the analyst's subjective reaction); reliability-qualifiers such as 'completely reliable'; and credibility-qualifiers such as 'possibly true'. Statements may also be as-

\footnotetext{
${ }^{124} \mathrm{http} / / / \mathrm{www}$. isi.edu/ikcap/trellis/
}

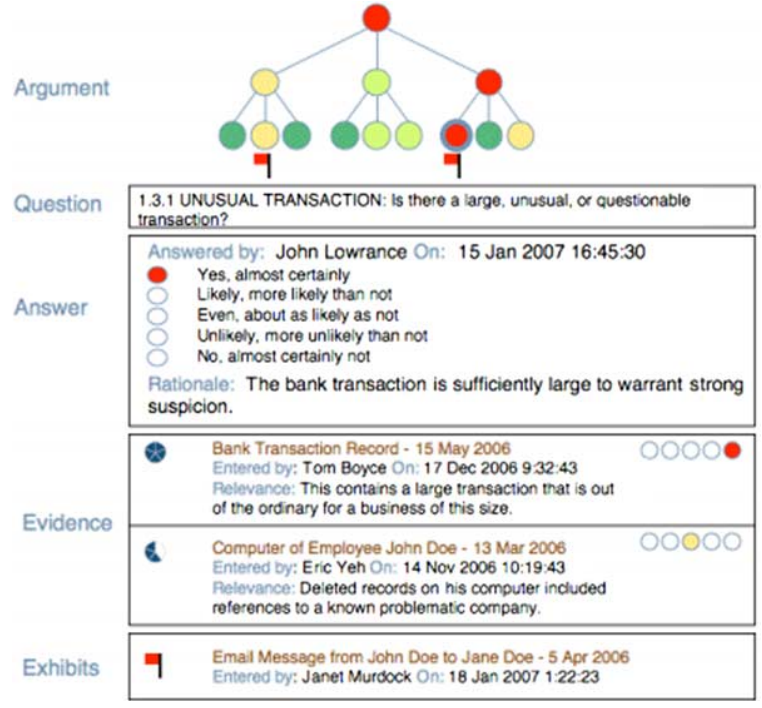

Fig. 52. SEAS uses a series of questions to structure the argument [113]. (Color figure online)

sociated with a document providing evidence. The source for each document, including creator, publisher, date, and format, is recorded. Originally, in Rich Trellis, users added rich relationships such as is elaborated by, is supported by, is summarized by, and stands though contradicted by, which the system stored in XML, RDF, and DAML+OIL.

In contrast to the detailed argumentation of Rich Trellis, Tree Trellis uses only pro and con, and collaborative discussion is supported, while Table Trellis 


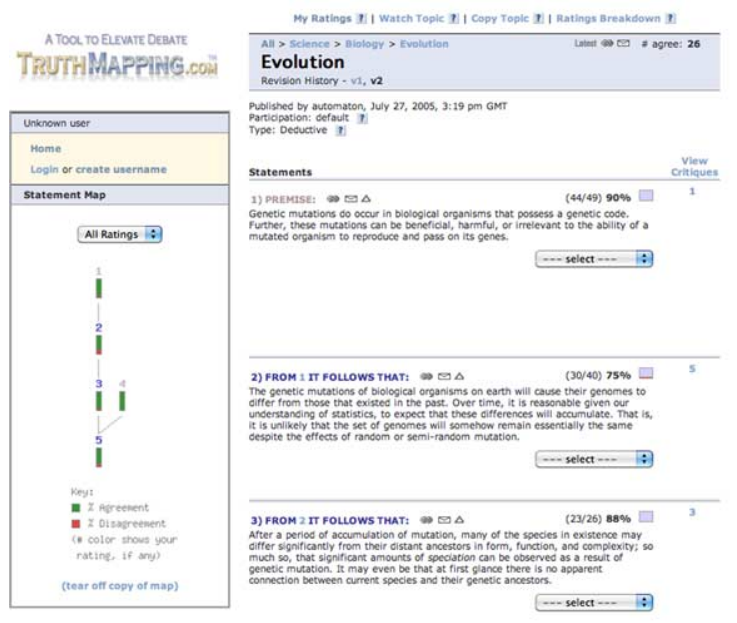

(a)

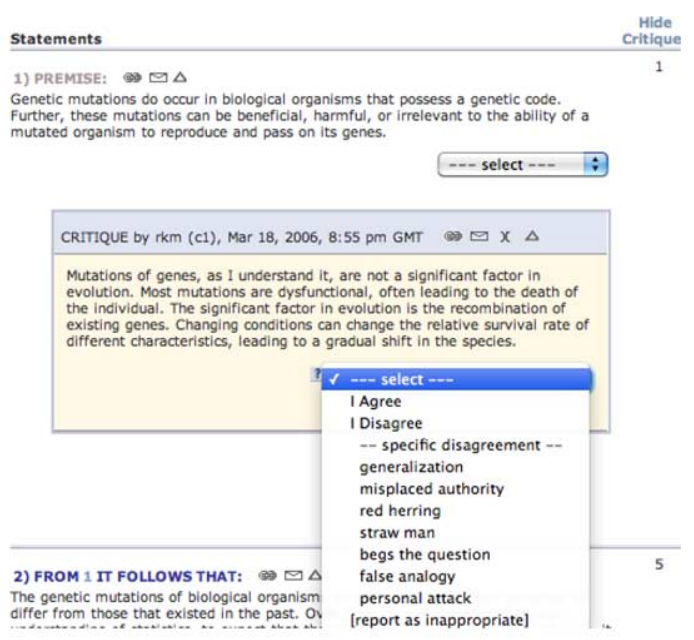

(b)

Fig. 53. Truthmapping (a) allows users to construct an argument by laying out premises and conclusions. Each node is colored based on the percentage of agreement (green) and disagreement (red). (b) Each premise and conclusion can be critiqued in comments, and critiques can be responded to with rebuttals. (Color figure online)

allows feature and value pairs to be arranged in a matrix, allowing the user to compare and evaluate alternatives according to their own criteria.

\section{A.36. TruthMapping}

TruthMapping $^{125}$ is an online deliberation tool which seeks to structure the conversation to focus around the "aha!" moment, avoiding digressions and soapboxes, and making hidden assumptions explicit. TruthMap facilitates structured conversations which use argument maps, critiques and rebuttals (Fig. 53(a)). Users can vote on and rate topics, and watch particular conversations Only one user, the original arguer, modifies the map; feedback comes in critiques attached to each premise and conclusion (Fig. 53(b)), which can be rebutted. One unique aspect of TruthMapping is that users can continually modify each contribution, but can only post one critique on each node. This is designed to make it easier to contribute a persistent comment to the discussion, which can not be drowned out by a single opponent. The system indicates when comments are out of sync, and a wiki-style history is available. Another unique aspect is the use of votes to color the map: as shown in Fig. 53(a), each node is colored based on the percentage of votes agreeing (green) and disagreeing (red).

\footnotetext{
${ }^{125} \mathrm{http}: / /$ www.truthmapping.com/
}

\section{A.37. Videolyzer}

Videolyzer ${ }^{126}$ [51] allows the general public to have sensemaking and argumentative discussions about the quality of online videos. It builds on gamelike-creation of video transcripts and on machine tagging of areas of interest in either the transcript (claim verbs, people, money, and comparison) or the video itself (faces) (Fig. 54(a) on page 214), to provide an integrated discussion forum for annotating and challenging the claims a video makes (Fig. 54(b) on page 214). Videolyzer is unique in its focus on integrating argumentative discussion into a video platform.

\section{B. Matrix comparison of tools}

We now present comparison charts of the tools reviewed. Figure 55 shows an overall comparison, in which tools are compared according to various features, which we outline shortly. For the downloadable tools, Fig. 56 on page 218 provides the license, programming language(s) and data storage. In both tables, we use '?' to indicate that we could not locate a piece of information.

First, we record the intended purpose of the tool. Next we provide the representation style and func-

\footnotetext{
${ }^{126} \mathrm{http}: / /$ videolyzer.com/
} 


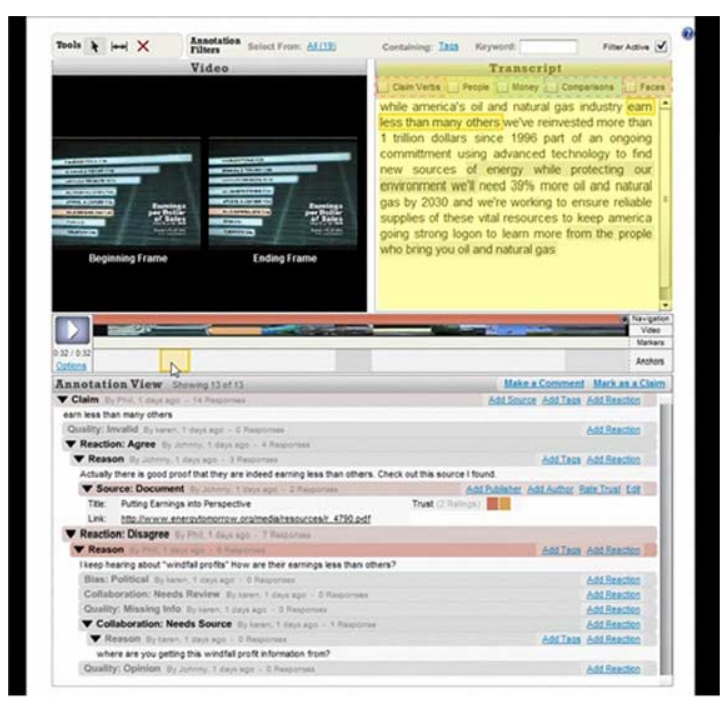

(a)

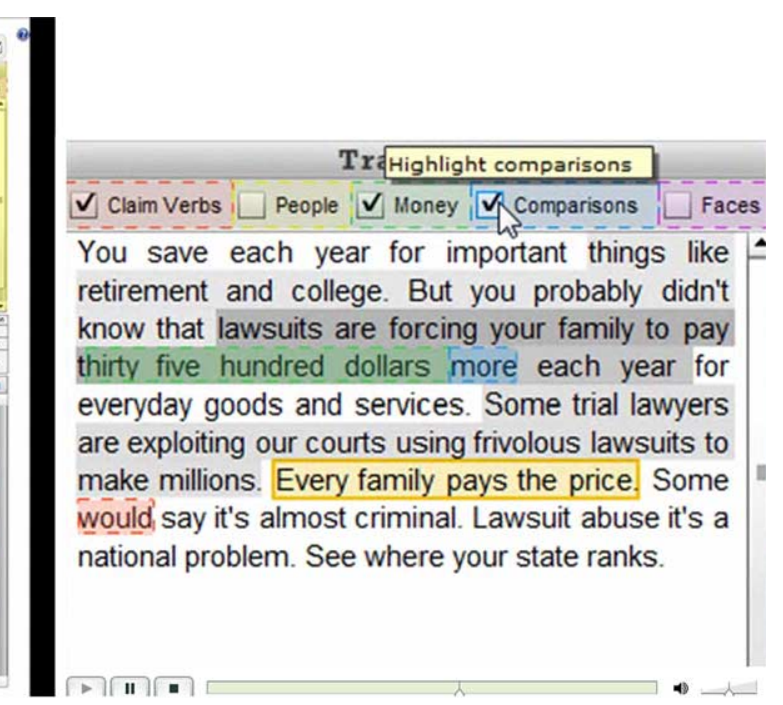

(b)

Fig. 54. Videolyzer (a) allows users to comment on the points made in a video; and (b) algorithmically determines segments of possible interest to help focus the discussion: in the transcript these are claim verbs and comparisons as well as mentions of people and money, and in the video these are peoples' faces. (Color figure online)

tional type. As introduced in Section 9.3, page 181, representation style is drawn from linear, threaded, graph, container, and matrix (including combinations of these styles); functional type is drawn from issue networking, funnelling, and reputation. Then we indicate what sort of advanced visualization is offered; '-' indicates that no examples were found (i.e. that the question does not apply). The perspective row records whether an individual user has a personal perspective distinct from the group view. Next we consider whether a tool has a distributed architecture (allowing multiple copies to synch with one another).

Then we distinguish downloadable and hosted systems (noting that some tools are in both categories or use a combined method). To understand their current integration with the Social Web, we record whether they use a site-specific login, or allow external credentials (such as OpenID, Twitter, or Facebook).

We further indicate whether they have any integration with third party services; a single row does not do justice to the wide range of integration we found. For tools with social networking capabilities, we provide an example of the interaction users can have with each other, or the information they can find out about each other. Stable URLs indicates our success in finding reusable bookmarks: in fact these URLs can be at multiple granularities, such as the entire argument map, issue, or conversation; each individual comment or critique; etc.

We also indicate, in the tags row, whether users can provide tags for content. We also indicate which tools have a bookmarklet for use while browsing, and which promote embedding on external sites. The remaining rows describe features related to the site's interaction style, starting with whether it is possible to attach media in discussions and the input type (such as point and click visual controls or formbased editing). We also indicate which have consistency checking (i.e. avoiding obvious contradictions) and credibility metrics (usually, but not always, voting) as well as export capabilities. Tools which export AIF can take advantage of an existing infrastructure.

Overall, we can make certain observations regarding these tools: generally they focus either on encouraging discussion or having a basis in rigorous argumentation models. Significant amounts of innovation has occurred in the research community, but many ideas have not been propagated to the Social Web at large. There are certain common mechanisms among many systems - basic features such as upvoting, segregating pro and cons, etc. Social Web systems do not have even levels of adoption: some tools are very well-adopted while others are not. 


\begin{tabular}{|c|c|c|c|c|c|c|}
\hline & ArgDF & ArgueHow & Argument Blogging & Argumentum & Argumentations.com & $\begin{array}{c}\text { AGORA: Participate- } \\
\text { Deliberate }\end{array}$ \\
\hline Purpose & $\begin{array}{l}\text { Create argumentation } \\
\text { schemes on the Web }\end{array}$ & $\begin{array}{c}\text { Distill the best points } \\
\text { to support position and } \\
\text { brace }\end{array}$ & $\begin{array}{l}\text { Enable argumentative } \\
\text { responses on the Web }\end{array}$ & Prove you're right & $\begin{array}{c}\text { Gather and use news } \\
\text { stories }\end{array}$ & Visualize debates \\
\hline Representation style & Text & Linear & Argument Map & Threaded & Threaded & Argument Map \\
\hline Functional type & Issue networking & Reputation & Issue networking & Reputation & Issue networking & Issue networking \\
\hline Advanced visualization & (from AIF) & - & - & - & Tag spheres & - \\
\hline Perspective & Single & Single & Single & Single & Single & Single \\
\hline Distributed architecture & $\mathrm{N}$ & $\mathrm{N}$ & $\mathrm{Y}$ & $\mathrm{N}$ & $\mathrm{N}$ & $\mathrm{N}$ \\
\hline Downloadable or hosted & Hosted, downloadable & Hosted & Combination & Hosted & Hosted & Combination \\
\hline Registration & Site-specific & Site-specific & No login & $\begin{array}{c}\text { Google, Facebook, } \\
\text { Twitter }\end{array}$ & Site-specific & - \\
\hline 3rd party services integration & - & $\begin{array}{l}\text { "Addthis" plugin for } \\
\text { sharing }\end{array}$ & $\begin{array}{l}\text { Blogs and publishing } \\
\text { platforms }\end{array}$ & $\begin{array}{c}\text { Argumentum Facebook } \\
\text { App }\end{array}$ & - & - \\
\hline Social networking capability & - & $\begin{array}{c}\text { User's comment } \\
\text { history } \\
\end{array}$ & - & Compare users & $\begin{array}{c}\text { User's comment } \\
\text { history }\end{array}$ & - \\
\hline Stable URLS & $?$ & $\mathrm{Y}$ & ? & $\mathrm{Y}$ & $\mathrm{Y}$ & - \\
\hline Tags & $\mathrm{N}$ & $\mathrm{N}$ & $\mathrm{N}$ & $\mathrm{N}$ & $Y$ & $\mathrm{~N}$ \\
\hline Bookmarklet & $\mathrm{N}$ & $\mathrm{N}$ & $\mathrm{Y}$ & $Y$ & $\mathrm{~N}$ & $\mathrm{~N}$ \\
\hline Promote embedding & $\mathrm{N}$ & $\mathrm{N}$ & $\mathrm{N}$ & $\mathrm{Y}$ & $\mathrm{N}$ & $\mathrm{N}$ \\
\hline Attach media & $\mathrm{N}$ & $\mathrm{N}$ & $\mathrm{Y}$ & $\mathrm{N}$ & $\mathrm{N}$ & $\mathrm{N}$ \\
\hline Input methods & Form-based & Form-based & HTML & $\begin{array}{c}\text { Form-based, depends } \\
\text { on type }\end{array}$ & $\begin{array}{c}\text { Form-based, depends } \\
\text { on type }\end{array}$ & Visual controls \\
\hline Consistency checking & Use AIF tools & $\mathrm{N}$ & $\mathrm{N}$ & $\mathrm{N}$ & $\mathrm{N}$ & $\mathrm{N}$ \\
\hline Credibility metrics & $\mathrm{N}$ & $Y$ & $\mathrm{~N}$ & $Y$ & $Y$ & $\mathrm{~N}$ \\
\hline Export formats & AlF & None & ? & None & None & None \\
\hline
\end{tabular}

(a)

\begin{tabular}{|c|c|c|c|c|c|c|}
\hline & Argunet & Avicenna & bCisiveOnline & Belvedere & $\begin{array}{c}\text { Cabanac's Annotation } \\
\text { System }\end{array}$ & Climate CoLab \\
\hline Purpose & $\begin{array}{l}\text { Sketch and share } \\
\text { argument maps }\end{array}$ & $\begin{array}{l}\text { Express arguments in } \\
\text { OWL to enable } \\
\text { classification }\end{array}$ & $\begin{array}{l}\text { Real-time collaboration } \\
\text { for decision-making }\end{array}$ & $\begin{array}{l}\text { Support secondary } \\
\text { school students learn } \\
\text { critical inquiry skills }\end{array}$ & $\begin{array}{c}\text { Sensemaking of } \\
\text { arguments in } \\
\text { annotations }\end{array}$ & $\begin{array}{l}\text { Collective intelligence } \\
\text { on climate change }\end{array}$ \\
\hline Representation style & Argument Map & Argument Map & Argument Map & Argument Map* & Threaded & Threaded \\
\hline Functional type & Issue networking & Issue networking & $\begin{array}{l}\text { Issue networking, } \\
\text { funelling }\end{array}$ & Issue networking & Funelling & Reputation \\
\hline Advanced visualization & Map associated items & - & - & Concept map, matrix & - & - \\
\hline Perspective & Personal & Single & Single & Single & Personal & Single \\
\hline Distributed architecture & $Y$ & $\mathrm{~N}$ & $\mathrm{~N}$ & $\mathrm{~N}$ & $\mathrm{~N}$ & $\mathrm{~N}$ \\
\hline Downloadable or hosted & Hosted, downloadable & Hosted & Hosted & Downloadable & Hosted & Hosted \\
\hline Registration & & Site-specific & Site-specific & - & Site-specific & Facebook \\
\hline 3rd party services integration & $\begin{array}{c}\text { "Addthis" plugin for } \\
\text { sharing }\end{array}$ & - & Skype & - & - & Facebook like \\
\hline Social networking capability & $\begin{array}{c}\text { Attribution but no user } \\
\text { profiles }\end{array}$ & - & Lists Skype usernames & - & - & Discussion, profiles \\
\hline Stable URLs & $\mathrm{Y}$ & $?$ & $\mathrm{Y}$ & $?$ & $\mathrm{~N}$ & $\mathrm{Y}$ \\
\hline Tags & $Y$ & $\mathrm{~N}$ & $\mathrm{~N}$ & $\mathrm{~N}$ & $\mathrm{~N}$ & $\mathrm{~N}$ \\
\hline Bookmarklet & $\mathrm{N}$ & $\mathrm{N}$ & $\mathrm{N}$ & $\mathrm{N}$ & $\mathrm{N}$ & $\mathrm{N}$ \\
\hline Promote embedding & $Y$ & $\mathrm{~N}$ & $\mathrm{Y}$ & $\mathrm{N}$ & $\mathrm{N}$ & $\mathrm{N}$ \\
\hline Attach media & $\mathrm{N}$ & $\mathrm{N}$ & $Y$ & Y & N & $\begin{array}{c}\text { Not in argument map } \\
\text { discussions }\end{array}$ \\
\hline Input methods & Visual controls & Visual controls & Visual controls & Visual controls & Visual controls & $\begin{array}{c}\text { Form-based, some } \\
\text { HTML and Wiki }\end{array}$ \\
\hline Consistency checking & $\mathrm{N}$ & Use AIF tools & $\mathrm{N}$ & $\mathrm{N}$ & $\mathrm{Y}$ & $\mathrm{N}$ \\
\hline Credibility metrics & $\mathrm{N}$ & $\mathrm{N}$ & $\mathrm{N}$ & $\mathrm{N}$ & $Y$ & $Y$ \\
\hline Export formats & Locally stored & AlF & PowerPoint & ? & ? & None \\
\hline
\end{tabular}

(b)

Fig. 55. Overall Comparison of Tools. 


\begin{tabular}{|c|c|c|c|c|c|c|}
\hline & Cohere & $\begin{array}{l}\text { Competing } \\
\text { Hypotheses }\end{array}$ & Consider It & ConviceMe & COPE_IT & CreateDebate \\
\hline Purpose & $\begin{array}{l}\text { Connect and share } \\
\text { ideas }\end{array}$ & $\begin{array}{l}\text { Analysis and cross- } \\
\text { checking }\end{array}$ & $\begin{array}{l}\text { Understand the } \\
\text { pros/cons behind your } \\
\text { opponents' opinions }\end{array}$ & Have fun debating & $\begin{array}{l}\text { e-Learning, } \\
\text { Collaboration }\end{array}$ & $\begin{array}{l}\text { K-12 education, } \\
\text { Debating }\end{array}$ \\
\hline Representation style & Argument Map & Matrix & Container & Container & $\begin{array}{c}\text { Argument Map, } \\
\text { Threaded }\end{array}$ & Container \\
\hline Functional type & Issue networking & Funelling & Issue networking & Reputation & Funelling & Reputation \\
\hline Advanced visualization & Map, timeline & $\begin{array}{c}\text { Sort by the most likely } \\
\text { hypotheses }\end{array}$ & $\begin{array}{l}\text { Show points according } \\
\text { to who holds them }\end{array}$ & - & $\begin{array}{c}\text { Automatically } \\
\text { formalize the view }\end{array}$ & $\begin{array}{c}\text { Summary graphs and } \\
\text { statistics }\end{array}$ \\
\hline Perspective & Personal & Personal & Single & Single & Personal & Single \\
\hline Distributed architecture & $\mathrm{N}$ & $\mathrm{Y}$ & $\mathrm{N}$ & $\mathrm{N}$ & $\mathrm{N}$ & $\mathrm{N}$ \\
\hline Downloadable or hosted & Hosted, downloadable & Hosted, downloadable & Hosted, downloadable & Hosted & Hosted, downloadable & Hosted \\
\hline Registration & Site-specific & Site-specific & Facebook & Facebook & OpenID & Site-specific \\
\hline 3rd party services integration & $\begin{array}{l}\text { RSS, Send messages to } \\
\text { Twitter }\end{array}$ & Find us on Twitter & $\begin{array}{c}\text { Find us on Facebook, } \\
\text { Twitter }\end{array}$ & $\begin{array}{l}\text { RSS, Send messages to } \\
\text { Facebook, Twitter }\end{array}$ & $\begin{array}{c}\text { Import phpNuke } \\
\text { webforums, } \\
\text { Compendium maps }\end{array}$ & $\begin{array}{c}\text { API, pull in external } \\
\text { RSS feeds, Facebook } \\
\text { fanpage }\end{array}$ \\
\hline Social networking capability & Groups, profiles & $\begin{array}{l}\text { Persistent chat, } \\
\text { message board }\end{array}$ & $\begin{array}{c}\text { Attribution, but no } \\
\text { user profiles }\end{array}$ & $\begin{array}{c}\text { Forum discussions, } \\
\text { user profiles }\end{array}$ & Groups & $\begin{array}{c}\text { List friends, and } \\
\text { enemies }\end{array}$ \\
\hline Stable URLs & $Y$ & $\mathrm{Y}$ & $\mathrm{Y}$ & $\mathrm{Y}$ & $\mathrm{N}$ & $\mathrm{Y}$ \\
\hline Tags & $Y$ & $\mathrm{~N}$ & $\mathrm{~N}$ & $\mathrm{Y}$ & $Y$ & $\mathrm{Y}$ \\
\hline Bookmarklet & $\mathrm{Y}$ & $\mathrm{N}$ & $\mathrm{N}$ & $\mathrm{N}$ & $\mathrm{N}$ & $\mathrm{Y}$ \\
\hline Promote embedding & $Y$ & $\mathrm{~N}$ & $\mathrm{~N}$ & $Y$ & $\mathrm{~N}$ & $Y$ \\
\hline Attach media & URLs only & N & $\mathrm{N}$ & N & Y & $\begin{array}{c}\text { URLs, embedded } \\
\text { videos only }\end{array}$ \\
\hline Input methods & Form-based & Form-based & Visual controls & Form-based & Form-based, WYSIWYG & Form-based, WYSIWYG \\
\hline Consistency checking & $\mathrm{N}$ & $\mathrm{Y}$ & $\mathrm{N}$ & $\mathrm{N}$ & $\mathrm{N}$ & $\mathrm{N}$ \\
\hline Credibility metrics & $\mathrm{N}$ & $\mathrm{N}$ & $\mathrm{N}$ & $Y$ & $\mathrm{~N}$ & $Y$ \\
\hline Export formats & None & None & None & None & None & None \\
\hline
\end{tabular}

(c)

\begin{tabular}{|c|c|c|c|c|c|c|}
\hline & Debate.org & Debategraph & Debatepedia & Debatewise & DiscourseDB & Dispute Finder \\
\hline Purpose & $\begin{array}{c}\text { Meet people through } \\
\text { debate }\end{array}$ & $\begin{array}{c}\text { Help groups } \\
\text { collaborate on } \\
\text { complex issues } \\
\end{array}$ & $\begin{array}{c}\text { Clarify public debate, } \\
\text { engage citizens }\end{array}$ & $\begin{array}{c}\text { Help make informed } \\
\text { decisions }\end{array}$ & $\begin{array}{c}\begin{array}{c}\text { Collect opinions of } \\
\text { commentators and } \\
\text { journalists }\end{array} \\
\end{array}$ & $\begin{array}{c}\text { Discover what's widely } \\
\text { disputed when } \\
\text { browsing the Web }\end{array}$ \\
\hline Representation style & Linear & $\begin{array}{l}\text { Argument Map, } \\
\text { Threaded }\end{array}$ & Container & Container & Container & - \\
\hline Functional type & Reputation & Issue networking & Issue networking & Reputation & Reputation & Reputation \\
\hline Advanced visualization & - & $\begin{array}{c}\text { Automatically change } \\
\text { the view }\end{array}$ & - & - & - & $\begin{array}{l}\text { Highlight disputed } \\
\text { sentences }\end{array}$ \\
\hline Perspective & Single & Single & Single & Single & Single & Personal \\
\hline Distributed architecture & $\mathrm{N}$ & $\mathrm{N}$ & $\mathrm{N}$ & $\mathrm{N}$ & $\mathrm{N}$ & $\mathrm{N}$ \\
\hline Downloadable or hosted & Hosted & Hosted & Hosted & Hosted & Hosted & Combination \\
\hline Registration & Site-specific & Site-specific & Site-specific & OpenID & Site-specific & No login \\
\hline 3rd party services integration & \begin{tabular}{|c|}
$\begin{array}{c}\text { Facebook like, Post to } \\
\text { Twitter, Send email }\end{array}$ \\
\end{tabular} & RSS, email & $\begin{array}{l}\text { RSS, post on Facebook, } \\
\text { Twitter, Delicious, Digg }\end{array}$ & $\begin{array}{l}\text { "Addthis" plugin for } \\
\text { sharing, Facebook like }\end{array}$ & - & Facebook \\
\hline Social networking capability & Extensive user profiles & User profiles & $\begin{array}{c}\text { User's contribution } \\
\text { history }\end{array}$ & - & $\begin{array}{c}\text { User's contribution } \\
\text { history }\end{array}$ & Profiles \\
\hline Stable URLS & $Y$ & $\mathrm{Y}$ & $\mathrm{Y}$ & $\mathrm{Y}$ & $Y$ & $\mathrm{~N}$ \\
\hline Tags & - & $\mathrm{N}$ & $\mathrm{N}$ & $\mathrm{N}$ & $\mathrm{N}$ & $\mathrm{N}$ \\
\hline Bookmarklet & $\mathrm{N}$ & $\mathrm{N}$ & $\mathrm{N}$ & $\mathrm{N}$ & $\mathrm{N}$ & $\mathrm{N}$ \\
\hline Promote embedding & $\mathrm{N}$ & $\mathrm{Y}$ & $\mathrm{N}$ & $\mathrm{N}$ & $\mathrm{N}$ & $\mathrm{N}$ \\
\hline Attach media & $\mathrm{N}$ & $\begin{array}{l}\text { URLs, embedded } \\
\text { videos only }\end{array}$ & Y & N & Y & $\mathrm{N}$ \\
\hline Input methods & Text with HTML & Form-based & Wiki formatting & Form-based, Wiki-style & Wiki formatting & $\begin{array}{c}\text { Roll-over text in user } \\
\text { mode, Form-based for } \\
\text { activist }\end{array}$ \\
\hline Consistency checking & $\mathrm{N}$ & $\mathrm{N}$ & $\mathrm{N}$ & $\mathrm{N}$ & $\mathrm{N}$ & $\mathrm{N}$ \\
\hline Credibility metrics & $Y$ & $\mathrm{~N}$ & $\mathrm{~N}$ & $Y$ & $\mathrm{~N}$ & $Y$ \\
\hline Export formats & None & None & None & None & RDF & None \\
\hline
\end{tabular}

(d)

Fig. 55. (Continued). 


\begin{tabular}{|c|c|c|c|c|c|c|}
\hline & HyperNews & LivingVoice & Opinion Space & $\begin{array}{c}\text { Online Visualization of } \\
\text { Arguments }\end{array}$ & Parmenides & PDOnline \\
\hline Purpose & $\begin{array}{c}\text { Argumentative Web } \\
\text { forum with an email } \\
\text { gateway }\end{array}$ & $\begin{array}{l}\text { "An up-to-date record } \\
\text { of what we believe and } \\
\text { why we believe it" }\end{array}$ & Exchange perspectives & $\begin{array}{l}\text { Analyze and diagram } \\
\text { arguments }\end{array}$ & $\begin{array}{c}\text { Get feedback on } \\
\text { proposals for } \\
\text { e-participation }\end{array}$ & $\begin{array}{l}\text { Speed scientific } \\
\text { communication }\end{array}$ \\
\hline Representation style & Threaded & Argument Map & - & Argument Map & Argument Map & Threaded \\
\hline Functional type & $\begin{array}{l}\text { Issue networking, } \\
\text { discussion }\end{array}$ & Issue networking & Reputation & Issue networking & Funelling & Reputation \\
\hline Advanced visualization & - & - & $\begin{array}{c}\text { Uses principal } \\
\text { component analysis }\end{array}$ & (from AIF) & $\begin{array}{c}\text { Analysis toolset uses } \\
\text { Value-based }\end{array}$ & - \\
\hline Perspective & Single & Single & Personal & Personal & Personal & Single \\
\hline Distributed architecture & $\mathrm{Y}$ & $\mathrm{N}$ & $\mathrm{N}$ & $\mathrm{N}$ & $\mathrm{N}$ & $\mathrm{N}$ \\
\hline Downloadable or hosted & Hosted, downloadable & Hosted & Hosted & Hosted & Hosted & Hosted \\
\hline Registration & Site-specific & Site-specific & Site-specific & No $\log$ in & No login & Site-specific \\
\hline 3rd party services integration & - & AddtoAny plugin & - & - & - & $\begin{array}{c}\text { Link to Facebook page, } \\
\text { Twitter }\end{array}$ \\
\hline Social networking capability & Discussion-based & $\begin{array}{c}\text { Vote on others' } \\
\text { opinions }\end{array}$ & Rate comments & - & - & Profiles, comments \\
\hline Stable URLs & $Y$ & $\mathrm{Y}$ & $\mathrm{N}$ & $Y$ & $Y$ & $Y$ \\
\hline Tags & $\mathrm{N}$ & $\mathrm{N}$ & $\mathrm{N}$ & $\mathrm{N}$ & $\mathrm{N}$ & $\mathrm{N}$ \\
\hline Bookmarklet & $\mathrm{N}$ & $\mathrm{N}$ & $\mathrm{N}$ & $Y$ & $\mathrm{~N}$ & $\mathrm{~N}$ \\
\hline Promote embedding & $\mathrm{N}$ & $\mathrm{N}$ & $\mathrm{N}$ & $\mathrm{N}$ & $\mathrm{N}$ & $\mathrm{N}$ \\
\hline Attach media & $\mathrm{N}$ & $\mathrm{N}$ & $\mathrm{N}$ & $\mathrm{N}$ & $\mathrm{N}$ & $Y$ \\
\hline Input methods & Form-based & Form-based & Form-based & Visual controls & Form-based & Form-based \\
\hline Consistency checking & $\mathrm{N}$ & $\mathrm{N}$ & $\mathrm{N}$ & Use AlF tools & $Y$, in admin view & $\mathrm{N}$ \\
\hline Credibility metrics & $\mathrm{N}$ & $Y$ & $Y$ & $\mathrm{~N}$ & $\mathrm{~N}$ & $Y$ \\
\hline Export formats & $?$ & None & None & ArgDB, AlF & ? & None \\
\hline
\end{tabular}

(e)

\begin{tabular}{|c|c|c|c|c|c|c|}
\hline & REASON & Riled Up! & SEAS & Trellis & TruthMapping & Videolyzer \\
\hline Purpose & $\begin{array}{l}\text { Support information } \\
\text { pooling }\end{array}$ & $\begin{array}{l}\text { Debate } \& \text { discuss; } \\
\text { show that you're an } \\
\text { authority on a topic }\end{array}$ & Intelligence analysis & Analysis & $\begin{array}{c}\text { Overcome "loudest } \\
\text { voice" and "last word" }\end{array}$ & $\begin{array}{c}\text { Collaboratively } \\
\text { evaluate online videos }\end{array}$ \\
\hline Representation style & $\begin{array}{c}\text { Argument Map, } \\
\text { Threaded }\end{array}$ & Container & Multiple & Linear & $\begin{array}{c}\text { Argument Map, } \\
\text { Threaded }\end{array}$ & Threaded \\
\hline Functional type & Funelling & Reputation & Funelling & Funelling & Funelling & Issue networking \\
\hline Advanced visualization & - & - & $\begin{array}{c}\text { Starburst, } \\
\text { Constellation }\end{array}$ & - & $\begin{array}{c}\text { Shows \% agreement } \\
\text { and disagreement }\end{array}$ & - \\
\hline Perspective & Personal & Single & Personal & Single & Single & Single \\
\hline Distributed architecture & $\mathrm{Y}$ & $\mathrm{N}$ & $\mathrm{Y}$ & $\mathrm{N}$ & $\mathrm{N}$ & $\mathrm{N}$ \\
\hline Downloadable or hosted & Hosted & Hosted & Hosted, downloadable & Downloadable & Hosted & Hosted \\
\hline Registration & Site-specific & Site-specific & Site-specific & Site-specific & Site-specific & Site-specific \\
\hline 3rd party services integration & - & $\begin{array}{c}\text { Digg, Delicious, Reddit, } \\
\text { Yahoo!, Google, } \\
\text { Stumble }\end{array}$ & - & - & - & - \\
\hline Social networking capability & Discussion & Profiles, comments & - & - & Discussion & Discussion \\
\hline Stable URLs & $\mathrm{N}$ & $\mathrm{Y}$ & $?$ & $?$ & $\mathrm{Y}$ & $\mathrm{Y}$ \\
\hline Tags & $\mathrm{N}$ & $\mathrm{Y}$ & $\mathrm{N}$ & $\mathrm{N}$ & $\mathrm{N}$ & $\mathrm{Y}$ \\
\hline Bookmarklet & $\mathrm{N}$ & $\mathrm{N}$ & $\mathrm{N}$ & $\mathrm{N}$ & $\mathrm{N}$ & $\mathrm{N}$ \\
\hline Promote embedding & $\mathrm{N}$ & $\mathrm{N}$ & $\mathrm{N}$ & $\mathrm{N}$ & $\mathrm{N}$ & $\mathrm{N}$ \\
\hline Attach media & $\mathrm{N}$ & $\mathrm{N}$ & $\mathrm{N}$ & URLs only & $\mathrm{N}$ & URLs only \\
\hline Input methods & Visual controls & Form-based & Form-based & Form-based & Form-based & Form-based \\
\hline Consistency checking & $\mathrm{N}$ & $\mathrm{N}$ & $?$ & $\mathrm{~N}$ & $\mathrm{~N}$ & $\mathrm{~N}$ \\
\hline Credibility metrics & $\mathrm{N}$ & Y & $\mathrm{Y}$ & $\mathrm{Y}$ & $Y$ & $Y$ \\
\hline Export formats & None & None & $\begin{array}{c}\text { Argument Markup } \\
\text { Language, HTML, Word }\end{array}$ & None & None & None \\
\hline
\end{tabular}

(f)

Fig. 55. (Continued) 


\begin{tabular}{|c|c|c|c|}
\hline & License & Language & Data storage \\
\hline ArgDF & $?$ & PHP & Sesame \\
\hline Argument Blogging & ? & $\begin{array}{l}\text { Python, Django, } \\
\text { Javascript, Jquery }\end{array}$ & AIFDB \\
\hline Argunet & Open source & Java & $\mathrm{Db} 40$ \\
\hline Avicenna & Author copyright & $\begin{array}{c}\text { Java with Jena, ARQ } \\
\text { Pellet }\end{array}$ & SQL Server DB \\
\hline bCisiveOnline & Commercial & Python, Django & $?$ \\
\hline Cohere & LGPL & PHP & MySQL \\
\hline Competing Hypotheses & GPL v3 & PHP5 & MySQL \\
\hline Considerlt & AGPL v3 & Ruby on Rails & $?$ \\
\hline CoPe_IT & Various & C\#, .NET & MS SQL Server 2005 \\
\hline Dispute Finder & Apache & $\begin{array}{c}\text { Python, Ruby, Scala, } \\
\text { Java }\end{array}$ & MS SQL Server \\
\hline HyperNews & MIT & Perl & Document directory \\
\hline SEAS & $\begin{array}{c}\text { Commercial, Free to } \\
\text { US Gov. }\end{array}$ & ? & (2) \\
\hline Trellis & GPL & Perl & $?$ \\
\hline
\end{tabular}

Fig. 56. Downloadable tools: License, language, and data storage. 